\title{
Acoustic Surface Cavitation
}

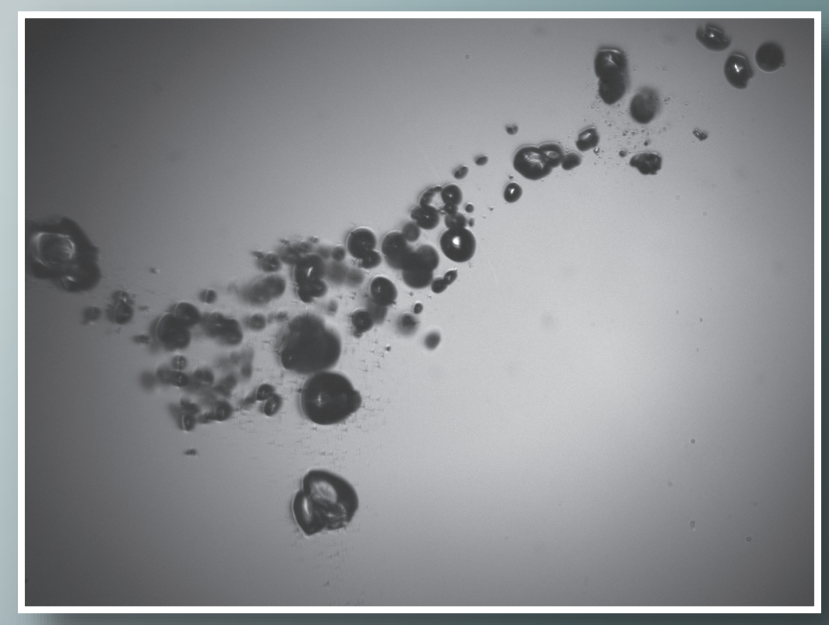

Aaldert Zijlstra 


\section{ACOUSTIC \\ SURFACE \\ CAVITATION}

Aaldert G. Zijlstra 


\section{Samenstelling promotiecommissie:}

Prof. dr. L. van Wijngaarden (voorzitter, secretaris) Universiteit Twente, TNW

Prof. dr. rer. nat. D. Lohse (promotor) Universiteit Twente, TNW

Dr. A. M. Versluis (assistent promotor) Universiteit Twente, TNW

Prof. dr. J. G. E. Gardeniers Universiteit Twente, TNW

Prof. dr. A. Prosperetti Universiteit Twente, TNW

Asst. Prof. dr. C.D Ohl Nanyang Technological University, Singapore

Dr. P. W. Mertens

IMEC vzw. Heverlee, Belgium

\section{imec \\ $+($}

This thesis was carried out at the Physics of Fluids group of the Faculty of Science and Technology of the University of Twente and the Ultra Clean Processing group of the Interuniversity Micro Electronics Center (IMEC), Leuven Belgium. This work is financially supported by IMEC vzw., Leuven, Belgium

\section{Cover:}

Acoustic cavitation bubbles adjacent to a silicon substrate (see Chapter 5).

\section{Dutch title:}

Akoestische Oppervlakte Cavitatie

\section{Publisher:}

Aaldert Geert Zijlstra, Physics of Fluids, University of Twente, P.O. Box 217, 7500 AE Enschede, The Netherlands http://pof.tnw.utwente.nl

a.g.zijlstra@alumnus.utwente.nl

Ph.D. Thesis, University of Twente

Printed by Gildeprint Drukkerijen

(C) Aaldert Geert Zijlstra, Enschede, The Netherlands 2011.

No part of this work may be reproduced by print, photocopy or any other means without the permission in writing from the publisher.

ISBN: 978-90-365-3241-9 


\section{ACOUSTIC SURFACE CAVITATION}

\section{PROEFSCHRIFT}

ter verkrijging van

de graad van doctor aan de Universiteit Twente, op gezag van de rector magnificus,

prof. dr. H. Brinksma, volgens besluit van het College voor Promoties in het openbaar te verdedigen op vrijdag 2 september 2011 om 16.45 uur

door

\section{Aaldert Geert Zijlstra}

geboren op 11 augustus 1977

te Groningen 
Dit proefschrift is goedgekeurd door de promotoren:

Prof. dr. rer. nat. D. Lohse

en de assistent promotor:

Dr. A. M. Versluis 


\section{Contents}

$\begin{array}{lll}1 & \text { Introduction } & 1\end{array}$

1.1 Bubbles localize and focus . . . . . . . . . . . . . . 1

1.2 The effects of cavitation . . . . . . . . . . . . . . . . . . 5

1.2 .1 Liquid motion . . . . . . . . . . . . . . . . 5

1.2 .2 Energy concentration . . . . . . . . . . . . . . 7

1.2 .3 Acoustic emission . . . . . . . . . . . . . . . . . . . 7

1.3 Applied acoustic cavitation . . . . . . . . . . . . . . . . . . . . 8

1.3.1 Megasonic Cleaning of semiconductor substrates . . . . 9

1.4 The origin of cavitation bubbles . . . . . . . . . . . . 12

1.5 Guide through the chapters . . . . . . . . . . . . . . . 13

2 Stability and acoustic response of a micropit bubble $\quad 19$

2.1 Introduction . . . . . . . . . . . . . . . . . . . . . . 19

2.2 A cylindrical micropit bubble . . . . . . . . . . . . . . 21

2.2 .1 Stability of the micropit bubble . . . . . . . . . . 23

2.3 Acoustic response of a micropit bubble . . . . . . . . . . . 28

2.3.1 The kinematic boundary conditions. . . . . . . . . 30

2.3 .2 The dynamic boundary conditions . . . . . . . . . . 31

2.4 Solution $\ldots \ldots \ldots \ldots \ldots$

2.4 .1 Solution with Fourier-Bessel series . . . . . . . . . . 34

2.5 Results . . . . . . . . . . . . . . . . . . . . . . . . . . . 36

2.5 .1 Effective mass and damping . . . . . . . . . 36

2.5 .2 Response of the micromeniscus . . . . . . . . . . . 36

2.6 Discussion and Conclusion . . . . . . . . . . . . . 41

3 Illumination by Laser-Induced Fluorescence for single flash imag$\begin{array}{ll}\text { ing on a nanoseconds timescale } & 45\end{array}$

3.1 Introduction . . . . . . . . . . . . . . . . . . . 45

3.2 Criteria for high-speed flash-photography . . . . . . . . . . 48

3.3 Illumination by laser induced fluorescence . . . . . . . . . . . 49

3.4 Comparison with other light sources $\ldots \ldots \ldots$. . . . . . . . 51

5.5 Examples . . . . . . . . . . . . . . . . . . . . . . . . . . . . . 54

3.6 Discussion and conclusion . . . . . . . . . . . . . . . . 58

4 Fiber optic probe hydrophone measurements in a cavitating liquid $^{1} 63$

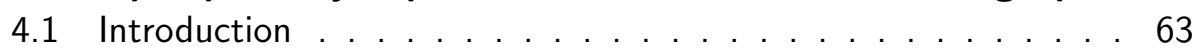

4.2 Experimental setup . . . . . . . . . . . . . . . . . . . 66

4.3 Results . . . . . . . . . . . . . . . . . . . . . . . . . . . . . . . . . . . 67 
4.4 Discussion \& conclusion . . . . . . . . . . . . . . . . . . . . . . . 69

5 Micropit bubble pinch-off $\quad 73$

5.1 Introduction . . . . . . . . . . . . . . . . . . 73

5.2 Materials \& methods . . . . . . . . . . . . . . 76

5.2 .1 Silicon micromachining . . . . . . . . . . . . 76

5.2 .2 The acoustic field . . . . . . . . . . . . . . . 76

5.2 .3 Imaging methods . . . . . . . . . . . . . . . . . 78

5.3 Results . . . . . . . . . . . . . . . . . . . . . . . . . . . 80

5.3 .1 Driving at $80 \mathrm{kHz} \ldots \ldots \ldots . \ldots . \ldots 80$

5.3 .2 Driving at $200 \mathrm{kHz} \ldots \ldots . \ldots . \ldots 83$

5.3 .3 Interaction of cloud cavitation . . . . . . . . . . 86

5.3 .4 Surface damage . . . . . . . . . . . . . . . . . . . 91

5.4 Discussion \& conclusion . . . . . . . . . . . . . . . . . . . . . 92

\begin{tabular}{|lll}
\hline 6 & Micropit bubble enhanced sonochemistry & 97
\end{tabular}

6.1 Introduction . . . . . . . . . . . . . . . . . . . 97

6.2 Experimental methods and materials . . . . . . . . . . . . 99

6.2 .1 Silicon micromachining: . . . . . . . . . . . . . . . . . . 99

6.2 .2 The acoustic field . . . . . . . . . . . . . . . . . . 99

6.2 .3 Luminol visualization and determination of the radical formation rate . . . . . . . . . . . . . . . 100

6.3 Results . . . . . . . . . . . . . . . . . . . . . . . . . 103

6.4 Discussion and conclusions . . . . . . . . . . . . . 106

$\begin{array}{lll}7 & \text { High-speed imaging of } 1 \mathrm{MHz} \text { surface acoustic cavitation } & 111\end{array}$

7.1 Introduction . . . . . . . . . . . . . 111

7.2 Experimental setup . . . . . . . . . . . . . . . . . 112

7.2 .1 The acoustic field . . . . . . . . . . . . . . . . 112

7.2 .2 The particle layer . . . . . . . . . . . . . . . . . 113

7.2 .3 Imaging methods . . . . . . . . . . . . . . . . . 114

7.3 Results . . . . . . . . . . . . . . . . . . . . . 116

7.3 .1 Particle removal . . . . . . . . . . . . . . . 116

7.3 .2 Cleaning bubble dynamics . . . . . . . . . . . . . 118

7.4 Discussion and conclusion . . . . . . . . . . . . . . . . . 121

$\begin{array}{lll}8 & \text { Conclusions and outlook } & 125\end{array}$

\begin{tabular}{ll}
\hline Summary & 128
\end{tabular}

\begin{tabular}{ll}
\hline Samenvatting & 132
\end{tabular} 
\begin{tabular}{ll}
\hline Acknowledgements & 136
\end{tabular}

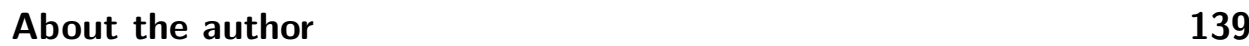





\section{1 \\ Introduction}

\subsection{Bubbles localize and focus}

Bubbles in liquids offer familiar sights and sounds in our daily lives. Just pour a soft drink, boil some water or open a water faucet and bubbles can readily be seen and heard. At first sight, bubbles might seem nothing more than simple discontinuities in an otherwise continuous liquid. What makes bubbles truly remarkable, however, is that it takes surprisingly little to transform these gently rising objects into miniature chemical reaction vessels, sources of light or even destroyers of ship propellers. These and other effects are associated with what is commonly referred to as cavitation; the formation of gas and/or vapor filled bubbles in a liquid under tension and their response to pressure variations.

There is a multitude of mechanisms that can bring about cavitation, for example decompression, liquid flow or impact of an object on the body of liquid. Another technique which is particularly simple and effective and therefore widely used, is the application of an acoustic field, notably ultrasound. As might be expected, acoustically induced bubble activity in a liquid is better known as acoustic cavitation [1-3]. A system in which this type of cavitation is produced typically consists of a liquid container and one or more piezoelectric transducers and some driving electronics. Depending on the properties of the liquid, the dimensions of the container and the acoustic parameters one can generate a certain distribution of bubbles displaying a wide variety of fascinating bubble dynamics phenomena. Bubbles subjected to the applied pressure field will be pushed around, deform, oscillate, collapse, coalesce with other bubbles, fragment into smaller bubbles, dissolve or grow. As will be discussed, pressure driven bubbles can bring forth a range of unique physical, chemical and biological effects that would be impossible to achieve solely by an acoustic field.

The principle lying behind all these phenomena is compressibility, the defining difference between a gas and a liquid. The volume of liquid hardly changes in response to a variation in pressure, whereas the volume of the gaseous interior 


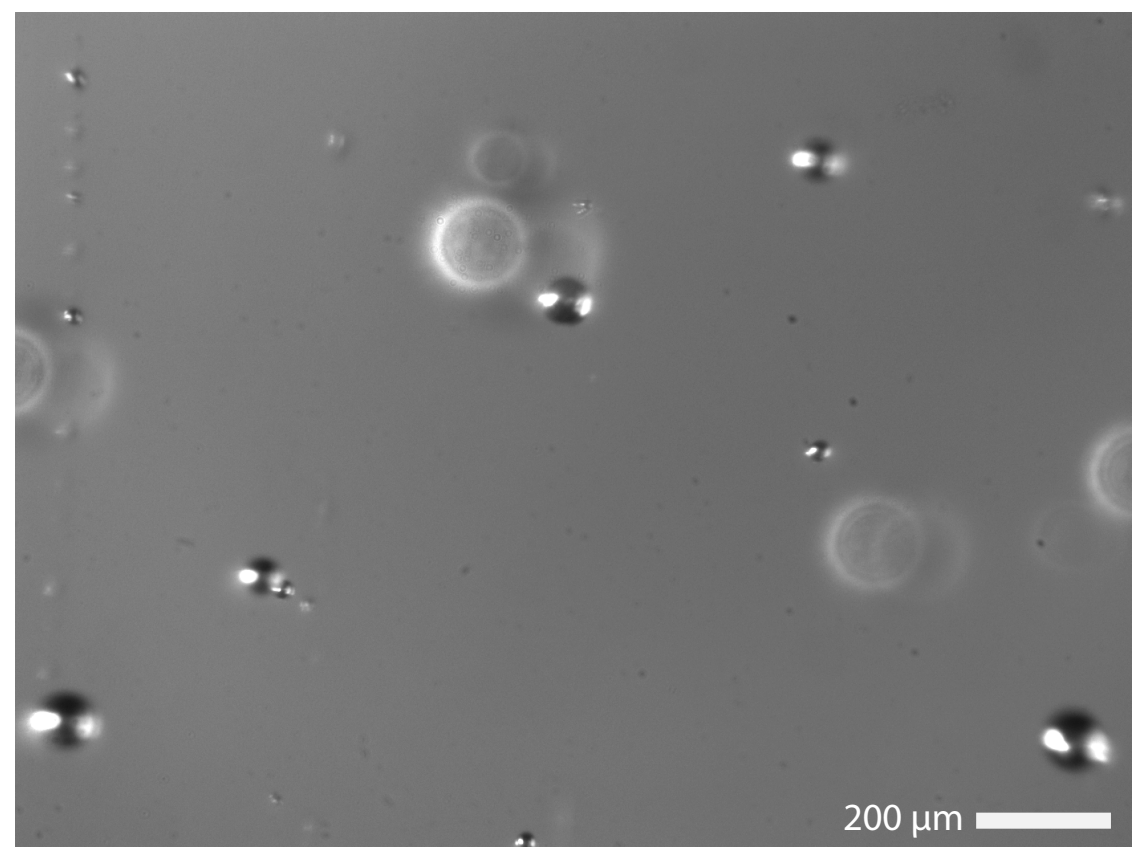

Figure 1.1: Cavitation bubbles in a $1 \mathrm{MHz}$ standing acoustic field.

of a bubble can change dramatically. Any contraction or expansion of the bubble is inevitably accompanied by a displacement of an equal volume of the much denser surrounding liquid. This gives rise to the remarkable property of acoustic cavitation that a large scale diffuse acoustic field can generate fluid motion which is localized at the positions of the bubbles within the liquid. Evidently, the extent of the volume changes and the subsequent fluid motion are determined by the amplitude and time scale of the pressure variations. Here, strong bubble response in combination with the compressible interior can provide not only localized fluid motion but, as will be discussed, also tremendous focusing of the liquid kinetic energy. It is precisely this mechanism which forms the basis of the more energetic and spectacular effects for which cavitation is best known.

To illustrate this focusing mechanism and its scale, it is instructive to examine the classic topic of bubble dynamics which is the response of a spherical bubble exposed to a pressure disturbance. Ever since Lord Rayleigh first studied the collapse of a spherical cavity in 1917 [4], a great body of work has been created on this subject. The reason for the continuing interest in spherical bubble dynamics is the fact that, in practice, many bubbles assume the shape of a sphere when the effect of surface tension is dominant. This turned out to be 
a rather fortuitous property as the spherical symmetry greatly facilitated the derivation of the central equation in bubble dynamics: the famous RayleighPlesset equation [1, 5]:

$$
R \ddot{R}+\frac{3}{2} \dot{R}^{2}=\frac{1}{\rho}\left(p_{b}-p_{0}-p_{\infty}(t)-4 \mu \frac{\dot{R}}{R}-\frac{2 \sigma}{R}\right) .
$$

This nonlinear equation expresses the relation between the radius of the bubble wall $R(t)$ and its time derivatives to the stresses acting on it. These stresses are due to the pressure inside the bubble $\left(p_{b}\right)$, the ambient pressure $\left(p_{0}\right)$, the driving pressure in the liquid $\left(p_{\infty}\right)$, the Newtonian liquid viscosity and surface tension. A closed form solution for this equation can be obtained only in a few special cases [2, 6], however numerical evaluation is straight forward. Over the years, the Rayleigh-Plesset equation was shown to provide rather accurate descriptions of the response of a bubble in many situations. To also accommodate effects such as liquid compressibility and thermal damping, a range of more advanced versions of the equation have been derived [7, 8]. The essential characteristics of spherical bubble dynamics are captured by the Rayleigh-Plesset equation however.

One such essential characteristic is the nonlinear response of a bubble which becomes more pronounced for increasing driving pressure. This is illustrated in Figure 1.2 showing the response of a microbubble in terms of its radius $\mathrm{R}$ subjected to a $50 \mathrm{kHz}$ sinusoidal driving pressure for two different amplitudes. The lowest amplitude (20 kPa, Figure 1.2(a)) causes small, close to sinusoidal deviations of the bubble wall in anti-phase with the driving pressure. Here, the maximum velocity of the bubble wall is approximately $5 \mathrm{~cm} / \mathrm{s}$. For this regime the bubble response closely resembles that of the classic harmonic oscillator. A characteristic of such a linear oscillatory system is that it possesses a resonance frequency. Indeed, from the linear approximation of the Rayleigh-Plesset equation $([1,2,5])$ a resonance frequency can be found which is given by

$$
f_{0}=\frac{1}{2 \pi R_{0}} \sqrt{\frac{1}{\rho}\left(3 \kappa p_{0}+(3 \kappa-1) \frac{2 \sigma}{R_{0}}\right)} .
$$

The pressure of the bubble interior is assumed to obey a polytropic law with $\kappa$ the polytropic exponent, $p_{0}$ is the ambient liquid pressure and $R_{0}$ is the equilibrium radius. Note that for our example bubble, the natural frequency is close to $1 \mathrm{MHz}$, much higher than the frequency with which it is driven.

Increased pressure amplitudes result in a completely different response as shown in Figure 1.2(b) for $120 \mathrm{kPa}$ amplitude. In this example, the bubble expands to about 4 times its equilibrium radius during the low pressure phase 

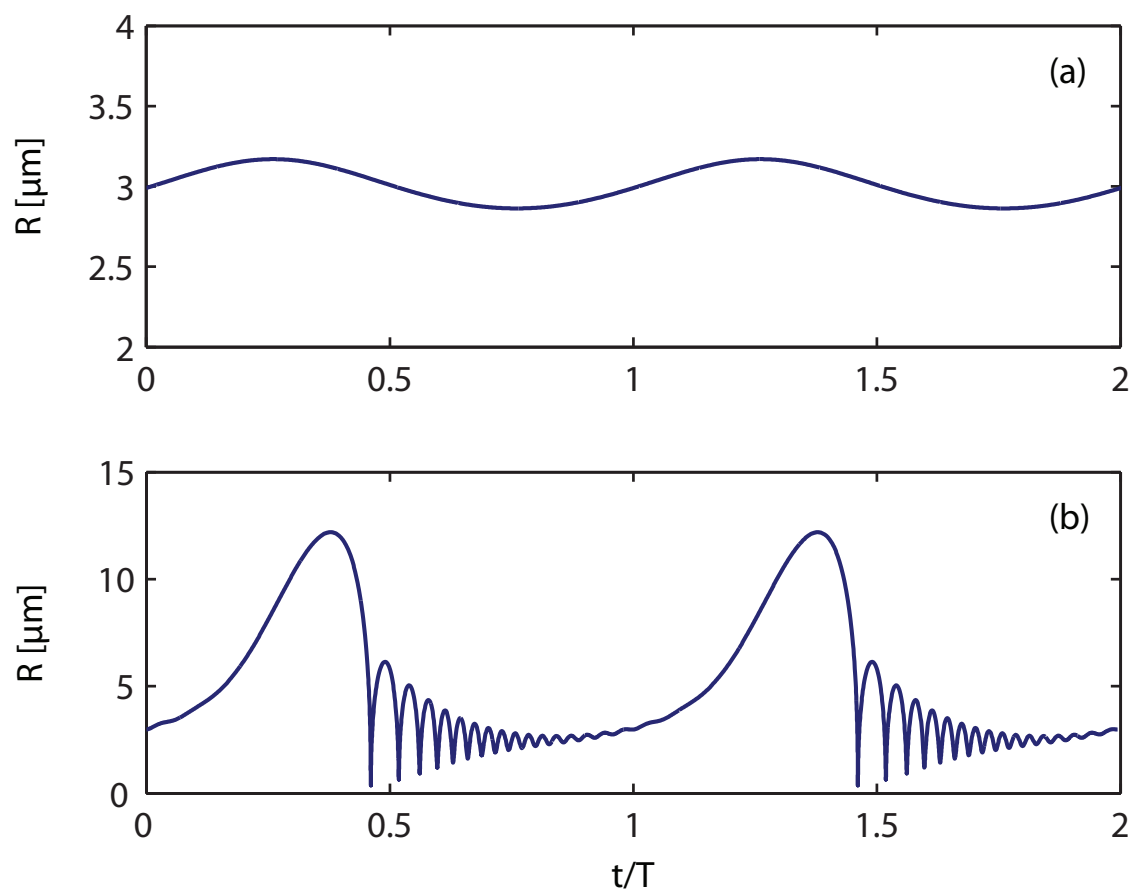

Figure 1.2: Two periods of the radial response of a spherical bubble obtained by numerical evaluation of the Rayleigh-Plesset equation. The bubble has an equilibrium radius of $3 \mu \mathrm{m}$ and is driven by $p_{\infty}(t)=-p_{A} \sin (2 \pi t / T)$ with $T=1 / f=20 \mu \mathrm{s}$. For low driving amplitudes (in this case $p_{A}$ of $20 \mathrm{kPa}(\mathrm{a})$ ), the response is close to linear and characterized by small oscillations. For larger amplitudes (here $120 \mathrm{kPa}$ (b)) the response reflects the strong non-linear character of the Rayleigh-Plesset equation.

of the acoustic cycle. When the pressure increases again the expansion is halted, creating similar conditions to what is known as the Rayleigh collapse 4]. Here, due to the large pressure imbalance the liquid accelerates inwards, thereby reaching velocities in the order of several hundreds of meters per second. At a certain point the bubble attains its equilibrium radius, but due to the gained momentum the liquid continues to compress the bubble even further. Eventually the pressure inside the bubble increases to such an extent that the flow is stopped and reversed, resulting in afterbounces.

These two regimes of the bubble oscillation exemplify two different types of acoustic cavitation. The regime characterized by low amplitude oscillations is referred to as non-inertial cavitation as the inertia of the liquid remains small. This in contrast with the regime showing high velocity bubble wall oscillations, appropriately referred to as inertial cavitation. The difference between the two regimes is made even clearer when considering the resulting kinetic energy 


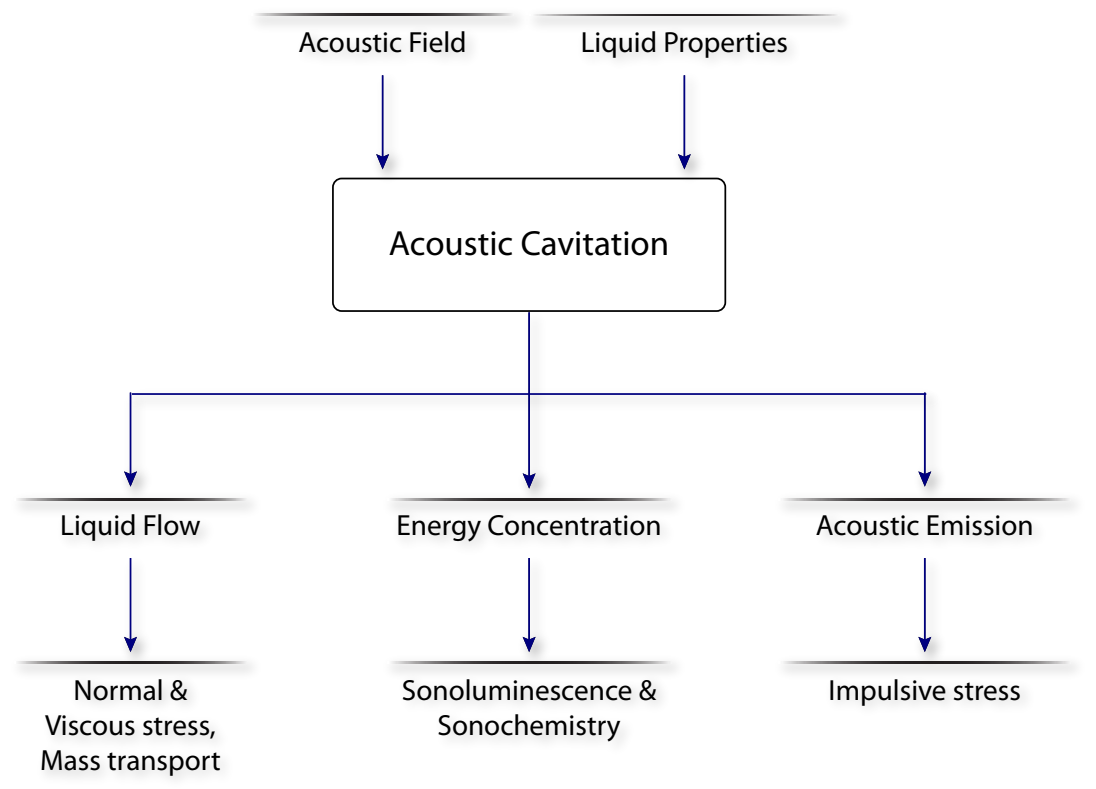

Figure 1.3: Schematic representation of acoustic cavitation and its effects.

densities, estimated using $e_{k}=\rho \dot{R}^{2}$. In the example, the maximum kinetic energy density for the low amplitude oscillations is approximately $2.5 \mathrm{~J} / \mathrm{m}^{3}$ while for the high amplitude oscillations a staggering $500 \mathrm{MJ} / \mathrm{m}^{3}$ is reached. Since the energy density of the acoustic field (given by $e_{a}=p^{2} / \rho c^{2}$, with c the speed of sound in water) in the last case was about $6 \mathrm{~J} / \mathrm{m}^{3}$ the tremendous energy focusing capability of a bubble is evident.

\subsection{The effects of cavitation}

Three interrelated primary effects of acoustic cavitation can be identified. These are (i) localized liquid motion, (ii) concentration of energy inside the bubble and (iii) acoustic emission.

\subsubsection{Liquid motion}

As discussed, liquid motion is inherent to the response of an acoustically driven bubble. Note that the oscillatory liquid flow need not be spherically symmetric however. Under certain conditions deviation from sphericity can occur due to surface instabilities [9, 10]. 
A more fundamental cause for loss of sphericity is the proximity of a symmetry breaking object, notably a solid wall or another bubble. The expansion and contraction of the bubble is now affected by the added resistance to flow due to the impermeability and the no-slip condition on the interface. This is particularly felt by the bubble wall closest to the interface while the opposite part of the bubble wall can move relatively less constrained. During the compression phase of the bubble this configuration causes the flow to converge mostly towards the axis of symmetry. This in turn can manifest itself in the form of a liquid jet directed through the bubble center towards the nearby object resulting in a toroidal shaped bubble. A frequently reproduced image of this phenomenon was made by Lawrence Crum for a millimeter sized bubble driven in a continuous $60 \mathrm{~Hz}$ pressure field [11]. It is common belief that liquid jet formation is also a generally occurring phenomenon for ultrasonic frequencies $(>20 \mathrm{kHz})$. This is supported by various numerical studies [12, 13], though experimental evidence is still limited. The reason for this is that bubbles in an acoustic field are notoriously difficult to study due to their small size, large mobility and short life times.

Only recently these difficulties have been circumvented with the use a bubble attached to a solid substrate driven with low ultrasonic frequencies [14-16]. The results of these experiments suggest that there exists a certain pressure threshold above which jet formation indeed can occur, as well as other types of more complicated bubble deformation and bubble pinch-off. Most of the existing knowledge on cavitation jets however results from numerical studies and experiments with reproducible spark or laser pulse generated bubbles, which are typically much larger in size (in the order of $1 \mathrm{~mm}$ ) [12, 17, 18]. In the transient collapse of these bubbles, jet velocities over 100 meters per second were found.

A less energetic type of liquid flow which can also be generated due to the proximity of a cavitation bubble to a solid-liquid interface is acoustic microstreaming [19-21]. This type of steady flow is essentially a manifestation of the non-zero average of the bubble induced oscillatory liquid motion, resulting from viscous dissipation mainly in the boundary layer of the nearby object. Acoustic microstreaming is typically circulation flow with velocities expressed in millimeters per second.

Any interface in contact with fluid motion will experience a certain amount of normal and tangential stress. Due to the properties of the liquid flow induced by acoustic cavitation, the thereby generated stress on an interface is intrinsically localized and can be impulsive, oscillatory and of high magnitude. In particular violent bubble collapse in contact with the wall is associated with impulsive pressures which may reach several GPa. Repeated exposure of the material 
to these forces can give rise to fatigue, deformation and eventually cavitation erosion [22]. It was in fact the erosive effect of hydrodynamic cavitation on ship propellers which initiated the research of pressure driven bubble dynamics [23]. Even though this was over a century ago, there is still no general agreement over the dominant mechanism responsible for surface damage occurring in any type of cavitation. Likely possibilities are high speed liquid impact onto the solid (e.g. jet flow) [13] and shock wave loading. Also the high pressure and temperatures developed in the bubble interior (see next sections) have been suggested to contribute to the effect [22].

The liquid motion induced by a cavitation bubble next to a surface can also give rise to considerable shear stress. For example, following the impact of a laser bubble generated cavitation jet, the liquid flow spreads out radially and parallel to the solid interface [24]. In the study of Dijkink et al. [25] a maximum shear stress of $3.5 \mathrm{kPa}$ was measured following jet impact. This indicates that at a height of only $1 \mu \mathrm{m}$ above the interface the liquid velocity was approximately $3.5 \mathrm{~m} / \mathrm{s}$. To put this in context: imposing the same amount of shear stress on the wall of a tube of $1 \mathrm{~cm}$ radius would require a practically impossible (turbulent) flow rate of about 130 liters per second! This example illustrates an essential feature of cavitation; bubbles are able to induce much stronger localized liquid motion in close proximity of a solid-liquid interface than attainable by macroscopic flow.

\subsubsection{Energy concentration}

The second effect of cavitation results from the conversion of kinetic energy into compression of the bubble contents. For the violent spherical bubble collapse, this happens so fast that there is no time for heat exchange with the surrounding liquid hence the volume change practically occurs adiabatically. The extreme compression leads to gas pressures of several hundreds of megapascals and temperatures rising to thousands of Kelvin. A so called "hot spot" is created within the cold liquid. These extreme conditions are short lived, yet have strong implications for the bubble constituents. Molecular excitation and dissociation catalyzes chemical reactions and results in the emission of photons. These intriguing high energetic effects brought forth by an acoustic field are referred to as sonochemistry [26] and sonoluminescence [27].

\subsubsection{Acoustic emission}

Bubbles essentially are secondary sources of sound, meaning that due to the bubble wall oscillation acoustic energy is re-emitted into the liquid. The nature of the sound emission is understandably coupled to the bubble dynamics. 
Small, close to linear bubble wall oscillations lead to likewise sound emissions while larger nonlinear oscillation gives rise to lower and higher harmonics [1]. The acoustic signature of cavitation often contains a significant broadband contribution as well, mostly attributed to shock waves resulting from strong inertial bubble collapse. If this collapse occurs close enough to a nearby object, the impinging shock wave may cause a certain amount of stress. This effect is thought to play an important role in cavitation erosion.

So far only the volumetric response of a bubble to the driving pressure was discussed, however bubbles also experience a translational force due to pressure gradients. The force resulting from the driving acoustic field is called the primary Bjerknes force [28]. Because bubbles produce a secondary acoustic field an additional secondary Bjerknes force is exerted on any bubble within this field [29, 30]. In proximity of an acoustic reflector, notably a solid wall, a bubble will also experience an attractive force due to the reflection of its own emitted acoustic field. It is the complex interplay of Bjerknes forces which determines the migration and spatial distribution of bubbles in any acoustic cavitation system.

\subsection{Applied acoustic cavitation}

From the perspective of engineers dealing with rapid liquid flow in machinery, cavitation is considered highly undesirable due to its destructive nature and therefore great effort is taken to prevent its occurrence. On the other hand, the properties of the more controllable acoustic cavitation provide unique conditions which are beneficial in many processes, witness the numerous applications in science, industry and in medicine.

The majority of these processes are mechanical in nature, exploiting the generated strong and local stresses on material interfaces within the cavitating liquid. Some examples are: mixing, from large volumes of liquid [31] to microscopic lab-on-a-chip environments [32], emulsification [33], cell disruption [34] and particle dispersion [35]. Another interesting biomedical technique is sonoporation [36], where increased cell permeability and therefore gene or drug uptake is achieved due to the stresses exerted by acoustically driven bubbles.

Perhaps the most established example of applied acoustic cavitation is ultrasonic cleaning. Here, the vigorous liquid motion in close proximity with the interface provides the drag forces required to dislodge and remove many types of contaminants from all sorts of objects. While the first patent on ultrasonic cleaning was filed during the second world war, industrial scale implementation began in the 1950's mostly for the cleaning of machine parts [37]. In the following decades more and more applications were found, from the cleaning 
of jewelry to window blinds, surgical instruments, root canals [38], laboratory equipments, textile [39], oil pipes, filter membranes [40], semiconductor substrates (see next section) and so on. Even though the technology is not new, it is thorough, fast, customizable and does not require the use of hazardous chemicals nor specialized and expensive equipment. These features make ultrasonic cleaning fit well within the current age of efficiency increase, cost reductions, and green technology.

Also the chemical effects of acoustic cavitation continue to receive plenty of attention from many researchers worldwide. The results of these studies demonstrate a multitude of unique and promising applications, in particular for the synthesis of new materials such as nanostructured metals, oxides and polymers [41]. It is therefore even more surprising that sonochemistry remains a rather uncommon processing technique in industry. Likely reasons for this are difficulties in upscaling the processes and low chemical conversion yields. Significant production of chemicals by means of cavitation typically requires a much larger energy consumption than for ultrasonic cleaning processes.

\subsubsection{Megasonic Cleaning of semiconductor substrates}

In the multi-billion dollar microelectronics industry great effort and expenses are incurred to prevent and remove even the smallest contaminants from semiconductor substrates. Contaminant films and particles, mostly originating from various processing steps, equipment and liquid containers are detrimental to the production of integrated circuits. Contamination related defects are estimated to be responsible for over $50 \%$ of loss of product yield, making cleaning of paramount importance. Of the almost thousand processing steps required for the production of an integrated circuit, about $20 \%$ are cleaning procedures [42]. Cleaning is achieved by a combination of chemical and physical techniques. Special chemical recipes have been developed with the purpose of decreasing the adhesive forces between the impurity and the substrate or to remove contaminants by etching. In most cases, additional physical forces are required to completely overcome the adhesion forces of the contaminants, especially for insoluble particles. Nowadays, the three main physical cleaning methods used in cleanrooms worldwide are: brush cleaning [43], spray cleaning [44] and megasonic cleaning.

The term megasonic cleaning was introduced in 1979 to differentiate the higher frequency range ultrasonic cleaning $(>700 \mathrm{kHz})$ from its lower frequency counterpart. At that time the general belief was that at these higher frequencies cavitation is non-existent so the cleaning mechanisms must be different. Cleaning was attributed to less violent mechanisms such as forcing of the acoustic 


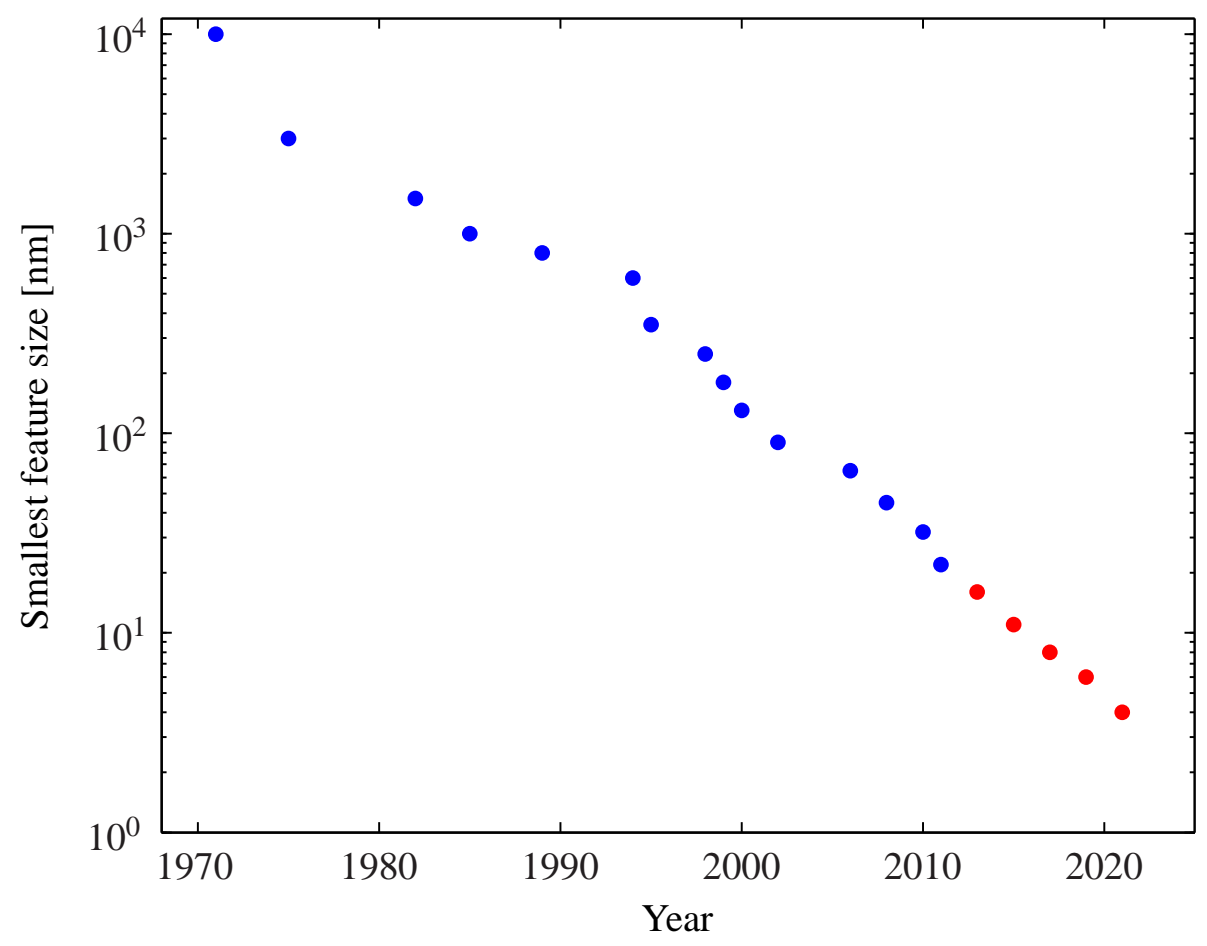

Figure 1.4: Historical (blue) and future (red) smallest feature size (technology node) in integrated electronic devices as stated in the international technology roadmap for semiconductors [42].

wave itself or secondary acoustic streaming effects and therefore highly suited for the cleaning of delicate semiconductor substrates [45, 46]. As megasonic cleaning emerged as a quick and effective cleaning method, there was no urgency in establishing the exact cleaning mechanism. A situation which lasted until the end of the twentieth century.

The semiconductor industry is one of the most innovative and fastest changing branches of technology. The main drive behind these innovations is the ever continuing downscaling of the integrated electronic structures (see Figure 1.4). This trend can only be sustained by continuous adaptations and redesigning of the processing methods, leading to new techniques such as immersion lithography. The drive to follow "Moore's Law" also had great implications for megasonic cleaning. After the introduction of microelectronic structures smaller than $130 \mathrm{~nm}$ (around the year 2000) it was discovered that even the seemingly gentle megasonic cleaning technique was causing feature damage [42]. Apparently the forces induced by traditional megasonic cleaning devices were sufficient to overcome both particle and structural adhesion forces. 
Particle removal of the substrates holding ever more fragile structures has become a serious challenge, not only for megasonic cleaning but for all available cleaning methods. This has two main reasons. First of all, if the feature dimensions decrease then also the size of the smallest particles which need to be removed decreases. For smaller particles, the ratio of the adhesion force versus the required hydrodynamic cleaning force increases thus making their removal more difficult. Secondly, due to changing environmental and health considerations as well as cost reductions and decreasing margins on the etching depth, the emphasis is shifting from chemical- to physical cleaning methods. As alternative cleaning methods are not readily available, it is clear that the existing techniques require substantial modifications in order to meet the increasing demands. Modifications which can only be made with sufficient knowledge of the cleaning mechanism.

At present, the exact mechanism of particle removal and damage by megasonic cleaning is still an open question. It is understood however that the direct forces of the acoustic field, either by drag forces or radiation pressure are insufficient to remove submicroscopic particles, let alone damage structures. Also acoustic streaming effects are unlikely to generate enough force to remove particles of $100 \mathrm{~nm}$ or less [47-50]. Only in the indirect acoustic effect of cavitation is the relatively low energy density of the acoustic field sufficiently focused.

Recently there have been several studies confirming the important role of cavitation bubbles in megasonic cleaning [47, 51]. Experiments also reveal that the effective cleaning range of single cavitation bubbles is typically of the same order as the bubble size [40, 47, 52, 53]. This suggests that effective cleaning requires many bubbles oscillating and moving in close contact with the substrate at acoustic intensities which result in removal of contaminants yet leave nanoscopic structures intact. The existence of such a process window has recently been demonstrated using lateral force measurements with an AFM device [54]. Therefore the question is: what are the physical forces exerted by acoustic cavitation bubbles and to what degree can these be controlled? It would make sense to simply reduce the acoustic pressure, however, this also diminishes nucleation and therefore the resulting bubble population. The same is true for increasing the frequency which decreases the resonant bubble size and would therefore decrease the spatial extent and forces exerted on the substrate. Clearly, the successful application of megasonic cleaning in the future not only depends on the ability to sufficiently control the location and the dynamics of acoustic cavitation bubbles but also their generation. 


\subsection{The origin of cavitation bubbles}

The preceding discussion raises a question on a fundamental issue: what is the origin of cavitation bubbles? In this context one usually refers to the pressure threshold for the inception, onset or nucleation of cavitation indicating the point from which on cavitation activity can be detected. There are basically two possibilities for this to occur. The first is called homogeneous nucleation where the liquid is ruptured when the tensile stress exceeds the intermolecular cohesion forces [55]. The tensile stresses associated with this type of nucleation are however much larger than found in most practical situations, and still, distributions of bubbles do appear, seemingly out of nowhere. The reason for this discrepancy is the presence of inhomogeneities in the liquid. Indeed, it is generally accepted that in any liquid not purified to an extreme measure, a second type of nucleation can occur which is classified as heterogeneous nucleation. In this case the pressure threshold is determined by the "weakest link" which is typically a pre-existing minute volume of gas [56]. Lack of mechanical and diffusive stability rules out that these gas volumes are free floating bubbles [57]. The most likely cavitation nucleus is therefore a bubble stabilized against dissolution inside a crevice in a solid surface as first proposed by Harvey et al. [58]. Microscopic and nanoscopic pits, crevices, indentations and such are believed to be present in virtually every solid object, notably the container wall but also the ubiquitous microparticles floating around in the liquid [59-61].

Similar to a free bubble, the response of a stabilized bubble will depend on its properties and on the frequency, amplitude and duration of the acoustic field to which it is exposed. Because the characteristics of these bubbles such as their geometries, sizes and locations are random and practically impossible to determine, very little is known about their acoustic response. Recently however, several studies were conducted on the dynamics of cavitation bubble nuclei which were artificially created using standard lithography techniques [56, 62, 63]. Pockets of air entrapped in cylindrical micropits varying from $50 \mathrm{~nm}$ to $20 \mu \mathrm{m}$ in radius were exposed to single pressure pulses with negative pressures down to $-3 \mathrm{MPa}$. The entrapped bubbles were observed to loose their mechanical stability and expand hemispherically outside the pit. The experiments by Borkent et al. [56] demonstrated that the threshold pressure for this to occur was in excellent agreement with the theoretically predicted quasistatic nucleation threshold by Atchley \& Prosperetti [64].

Since one typically finds distributions of many free bubbles in an acoustic cavitation field for a wide range of frequencies the following question arises: how does such a nucleated bubble end up in the bulk of the liquid? In literature the focus is mostly on nucleation thresholds and its detection. However, 
the mechanism leading to the development of bubble distributions seems to be overlooked or considered synonymous with nucleation. Clearly there must be some kind of bubble fragmentation or pinch-off mechanism for bubbles to populate the liquid volume. Also the role of the driving frequency in bubble nucleation is unclear as there are virtually no studies on the dynamic response of a crevice bubble to a continuous acoustic field.

\subsection{Guide through the chapters}

In the preceding sections several issues were raised concerning acoustic cavitation. As discussed, spherical bubble dynamics is a well developed field of research, yet practical applications based on acoustic cavitation rely mostly on bubbles oscillating in close proximity to solid surfaces where these bubbles typically deviate from sphericity. Also the initiation of cavitation activity is generally believed to be related to the response of microbubbles entrapped inside crevices in a solid surface. Little is known about the exact nature of this response however, neither theoretically nor experimentally. In this thesis the focus is therefore on the dynamics of bubbles either entrapped inside or moving near solid surfaces. In the next chapter, a model based on the unsteady Stokes equation for the linear response of a cylindrical micropit bubble subjected to a continuous acoustic field will be derived and investigated. Cavitation typically involves fast moving and microscopically small bubbles thus requiring highresolution imaging methods both in space and time. For this purpose a novel ultrashort illumination method is presented and tested in chapter 3 . In chapters 4 and 5 this flash-illumination method is applied, first for the investigation of the effect of cavitation induced shock waves on the measurement signal of a fiber optic hydrophone. Next, in chapter 5 the method is applied in the experimental investigation of the response of cylindrical micropit bubbles (artificial crevice bubbles) to a continuous ultrasound field. Here it is shown that the presence of (artificial) crevice bubbles greatly enhances cavitation activity. This property is exploited in the study of chapter 6 , where it is demonstrated that enhanced cavitation activity due to the presence of micropits significantly increases sonochemical efficiency. Finally, in chapter 7 , the dynamics of surface acoustic cavitation bubbles in relation to ultrasonic cleaning at $1 \mathrm{MHz}$ is investigated using high-speed imaging methods. Here the cleaning effect is directly linked to the dynamics of a single acoustic cavitation bubble. 


\section{Bibliography}

[1] T. G. Leighton. The Acoustic Bubble. Academic Press, London, 1994. ISBN 0124419208.

[2] C. E. Brennen. Cavitation and bubble dynamics. Oxford University Press, New York, 1995. ISBN 0195094093.

[3] R. E. Apfel. Sonic effervescence: A tutorial on acoustic cavitation. J. Acoust. Soc. Am., 101(3):1227, 1997.

[4] Lord Rayleigh. On the pressure developed in a liquid during the collapse of a spherical cavity. Philos. Mag., 34:9498, 1917.

[5] M. S. Plesset and A. Prosperetti. Cavitation and bubble dynamics. Ann. Rev. Fluid Mech., 9(1):145-185, 1977.

[6] J. P. Franc and J. M. Michel. Fundamentals of cavitation. Kluwer Academic Publishers, 2004.

[7] A. Lezzi and A. Prosperetti. Bubble dynamics in a compressible liquid. part 2. second-order theory. J. Fluid Mech., 185:289-321, 1987.

[8] A. Prosperetti. The thermal behavior of oscillating gas bubbles. J. Fluid Mech., 587(222):587-616, 1991.

[9] S. Hilgenfeldt, D. Lohse, and M. P. Brenner. Phase diagrams for sonoluminescing bubbles. Physics of Fluids, 8(11):2808, 1996.

[10] M. Versluis, D. E. Goertz, P. Palanchon, I. L. Heitman, S. M. van der Meer, B. Dollet, N. de Jong, and D. Lohse. Microbubble shape oscillations excited through ultrasonic parametric driving. Phys. Rev. E, 82(2):026321, 2010.

[11] L. A. Crum. Surface oscillations and jet development in pulsating bubbles. Le Journal de Physique Colloques, 40(C8):285-288, 1979.

[12] J. R. Blake and D. C. Gibson. Cavitation bubbles near boundaries. Ann. Rev. Fluid Mech., 19(1):99-123, 1987.

[13] E. A. Brujan. The role of cavitation microjets in the therapeutic applications of ultrasound. Ultrasound Med. Biol., 30(3):381 - 387, 2004. 
[14] S. Nakatani, K. Yoshida, and Y. Watanabe. Optical and acoustic observations of bubble adhered to piezoelectric transducer under ultrasound field: Pressure signal caused by bubble behavior. Japanese Journal of Applied Physics, 47(5):3904-3907, 2008.

[15] W. Kim, K. Park, J. Oh, J. Choi, and H. O. Kim. Visualization and minimization of disruptive bubble behavior in ultrasonic field. Ultrasonics, 50(8):798 - 802, 2010.

[16] F. Prabowo and C. D. Ohl. Surface oscillation and jetting from surface attached acoustic driven bubble. Ultrason. Sonochem., 18(1):431 - 435, 2011.

[17] C. Naude and A. Ellis. On the mechanism of cavitation damage by nonhemispherical cavities in contact with a solid boundary. Trans. ASME J. Basic Eng., 84(83):648656, 1961.

[18] W. Lauterborn and H. Bolle. Experimental investigations of cavitationbubble collapse in the neighbourhood of a solid boundary. J. Fluid Mech., 72(02):391-399, 1975.

[19] S. A. Elder. Cavitation Microstreaming. J. Acoust. Soc. Am., 31(1):54, 1959.

[20] B. Davidson and N. Riley. Cavitation microstreaming. J. Sound Vib., 15 (2):217-233, 1971.

[21] P. Marmottant and S. Hilgenfeldt. Controlled vesicle deformation and lysis by single oscillating bubbles. Nature, 423(6936):153-155, 2003.

[22] A. Philipp and W. Lauterborn. Cavitation erosion by single laser-produced bubbles. J. Fluid Mech., 361:75-116, 1998.

[23] C. A. Parsons and S. S. Cook. Investigations into the causes of corrosion or erosion of propellers. Journal of the American Society for Naval Engineers, 31(2):536-541, 1919.

[24] C. D. Ohl, M. Arora, R. J. Dijkink, V. Janve, and D. Lohse. Surface cleaning from laser-induced cavitation bubbles. Appl. Phys. Lett., 89(7): 074102, 2006.

[25] R. J. Dijkink and C. D. Ohl. Measurement of cavitation induced wall shear stress. Appl. Phys. Lett., 93(25):254107, 2008.

[26] K. S. Suslick. Sonochemistry. Science, 247(4949):pp. 1439-1445, 1990. 
[27] M. P. Brenner, S. Hilgenfeldt, and D. Lohse. Single-bubble sonoluminescence. Rev. Mod. Phys., 74(2):425-484, 2002.

[28] A. Eller. Force on a bubble in a standing acoustic wave. J. Acoust. Soc. Am., 43(1):170-171, 1968.

[29] L. A. Crum and A. I. Eller. Motion of bubbles in a stationary sound field. J. Acoust. Soc. Am., 48:181-189, 1970.

[30] L. A. Crum. Bjerknes forces on bubbles in a stationary sound field. J. Acoust. Soc. Am., 57(6):1363-1370, 1975.

[31] E. A. Vasil'tsov and A. Y. Isakov. Cavitation in mixing apparatus. Chemical and Petroleum Engineering, 11:1082-1084, 1975.

[32] R. H. Liu, J. Yang, M. Z. Pindera, M. Athavale, and P. Grodzinski. Bubbleinduced acoustic micromixing. Lab on a Chip, 2:151-157, 2002.

[33] C. Bondy and K. Sollner. On the mechanism of emulsification by ultrasonic waves. Trans. Faraday Soc., 31:835-843, 1935.

[34] J. E. Kennedy. High-intensity focused ultrasound in the treatment of solid tumours. Nature Reviews Cancer, 5(4):321-327, 2005.

[35] V. M. Fridman. The interaction mechanism between cavitation bubbles and particles of the solid and liquid phases. Ultrasonics, 10(4):162 - 165, 1972.

[36] A. van Wamel, K. Kooiman, M. Harteveld, M Emmer, ten F. J. Cate, M. Versluis, and N. de Jong. Vibrating microbubbles poking individual cells: Drug transfer into cells via sonoporation. Journal of Controlled Release, 112(2):149 - 155, 2006.

[37] T. J. Bulat. Macrosonics in industry: 3. ultrasonic cleaning. Ultrasonics, 12(2):59-68, 1974.

[38] M. Ahmad, T. R. Pitt Ford, and L. A. Crum. Ultrasonic debridement of root canals: Acoustic streaming and its possible role. J. Endod., 13(10): 490 - 499, 1987.

[39] V. S. Moholkar, M. C. G. Warmoeskerken, C. D. Ohl, and A. Prosperetti. Mechanism of mass-transfer enhancement in textiles by ultrasound. AIChE Journal, 50(1):58-64, 2004. 
[40] M. O. Lamminen, H. W. Walker, and L. K. Weavers. Mechanisms and factors influencing the ultrasonic cleaning of particle-fouled ceramic membranes. J. Membr. Sci., 237(1-2):213 - 223, 2004.

[41] J. H. Bang and K. S. Suslick. Applications of ultrasound to the synthesis of nanostructured materials. Advanced Materials, 22(10):1039-1059, 2010.

[42] K. A. Reinhardt and W. Kern. Handbook of silicon wafer cleaning technology. William Andrew Inc., 2008. ISBN 978-0-8155-1554-8.

[43] K. Xu, R. Vos, G. Vereecke, G. Doumen, W. Fyen, P. W. Mertens, M. M. Heyns, C. Vinckier, J. Fransaer, and F. Kovacs. Fundamental study of the removal mechanisms of nano-sized particles using brush scrubber cleaning. J. Vac. Sci \&Tech. B, 23(5):2160-2175, 2005.

[44] H. Hirano, K. Sato, T. Osaka, H. Kuniyasu, and T. Hattori. Damagefree ultradiluted hf/nitrogen jet spray cleaning for particle removal with minimal silicon and oxide loss. Electrochem. Solid-State Lett., 9(2):G62G65, 2006.

[45] A. Mayer and S. Shwartzman. Megasonic cleaning: A new cleaning and drying system for use in semiconductor processing. J. Electron. Mater., 8: 855-864, 1979.

[46] W. Kern. The evolution of silicon wafer cleaning technology. J. Electrochem. Soc., 137(6):1887-1892, 1990.

[47] W. Kim, T. H. Kim, J Choi, and H. Y. Kim. Mechanism of particle removal by megasonic waves. Appl. Phys. Lett., 94(8):081908-3, 2009.

[48] P. A. Deymier, A. Khelif, B. Djafari-Rouhani, J. O. Vasseur, and S. Raghavan. Theoretical calculation of the acoustic force on a patterned silicon wafer during megasonic cleaning. Appl. Phys. Lett., 88(5):2423-2429, 2000 .

[49] P. A. Deymier, J. O. Vasseur, A. Khelif, and S. Raghavan. Second-order sound field during megasonic cleaning of patterned silicon wafers: Application to ridges and trenches. Appl. Phys. Lett., 90(8):4211-4218, 2001.

[50] M. Olim. A theoretical evaluation of megasonic cleaning for submicron particles. J. Electrochem. Soc., 144(10):3657-3659, 1997.

[51] G. Vereecke, F. Holsteyns, S. Arnauts, S. Beckx, P. Jaenen, K. Kenis, M. Lismont, M. Lux, R. Vos, J. Snow, and P. W. Mertens. Evaluation of 
Megasonic Cleaning for Sub-90nm Technologies. Solid State Phenomena, 7(103-104):141-146, 2005.

[52] A. Thiemann, T. Nowak, R. Mettin, F. Holsteyns, and A. Lippert. Characterization of an acoustic cavitation bubble structure at $230 \mathrm{khz}$. Ultrason. Sonochem., 18(2):595 - 600, 2011.

[53] W. Kim, K. Park, J. Oh, J. Choi, and H. O. Kim. Visualization and minimization of disruptive bubble behavior in ultrasonic field. Ultrasonics, 50(8):798 - 802, 2010.

[54] T.-G. Kim, K. Wostyn, T. Beard, J.-G. Park, P. W. Mertens, and M. Heyns. Investigation of physical cleaning process window by atomic force microscope. ECS Transactions, 25(5):203-210, 2009.

[55] F. Caupin and E. Herbert. Cavitation in water: a review. Comptes Rendus Physique, 7(9-10):1000 - 1017, 2006.

[56] B. M. Borkent, S. Gekle, A. Prosperetti, and D. Lohse. Nucleation threshold and deactivation mechanisms of nanoscopic cavitation nuclei. Physics of Fluids, 21(10):102003, 2009.

[57] P. S. Epstein and M. S. Plesset. On the stability of gas bubbles in liquid-gas solutions. The Journal of Chemical Physics, 18(11):1505-1509, 1950.

[58] E. N. Harvey, D. K. Barnes, W. D. McElroy, A. H. Whiteley, D. C. Pease, and K. W. Cooper. Bubble formation in animals. i. physical factors. J. Cell. Comp. Physiol., 24(1):1-22, 1944.

[59] M. Strasberg. Onset of ultrasonic cavitation in tap water. J. Acoust. Soc. Am., 31(2):163-176, 1959.

[60] R. E. Apfel. The role of impurities in cavitation-threshold determination. J. Acoust. Soc. Am., 48(5B):1179-1186, 1970.

[61] R. E. Apfel. Acoustic cavitation inception. Ultrasonics, 22(4):167 - 173, 1984.

[62] N. Bremond, M. Arora, C. D. Ohl, and D. Lohse. Cavitation on surfaces. Journal of Physics: Condensed Matter, 17(45):S3603, 2005.

[63] N. Bremond, M. Arora, C. D. Ohl, and D. Lohse. Controlled multibubble surface cavitation. Phys. Rev. Lett., 96(22):224501, 2006.

[64] A. A. Atchley and A. Prosperetti. The crevice model of bubble nucleation. J. Acoust. Soc. Am., 86(3):1065-1084, 1989. 


\section{2}

\section{Stability and acoustic response of a micropit bubble}

In this chapter both the stability and the acoustic response of a model crevice bubble are considered. The bubble consists of an entrapped pocket of gas inside a cylindrical micropit immersed in an incompressible viscous liquid. A mechanical and diffusive balance suggests that micropit bubbles with radii smaller than a maximum radius, which depends on the dissolved gas concentration, can remain stable inside an isothermal stagnant liquid. The dynamic response of the bubble to a continuous small amplitude sinusoidal driving pressure is obtained from the solution for the unsteady Stokes equation combined with a Fourier-Bessel expansion for the axisymmetric liquid-gas interface. The model for the response of the circular liquid-gas interface is similar to a classic damped harmonic oscillator with the added feature of surface mode oscillations.

\subsection{Introduction}

A liquid insonified with ultrasound of sufficient intensity can develop a population of strongly interacting bubble of various sizes. As discussed in the previous chapter, the nature of these bubbles makes experimental investigation extremely difficult. Methods such as the use of very viscous liquids, optical tweezers [1] or carefully tuned standing-wave fields can be applied to overcome these difficulties. A prime example of the latter is the single bubble sonoluminescence setup [2, 3] allowing detailed investigation of a single bubble for a large parameter space. Another technique which has not received much attention until recently is to stabilize and immobilize one or more bubbles inside cavities micromachined into a solid object. When immersed in a liquid, air is entrapped within these cavities forming a stable liquid-gas micromeniscus. This system basically constitutes a model for the crevice bubble which is the centerpiece 
of heterogeneous nucleation theory [4, 5]. Indeed, recently Borkent et. al [6] studied the nucleation threshold of a crevice bubble for a single pressure pulse using nanoscopic micropits etched in silicon substrates.

The first to use artificial crevice bubbles were Hughes \& Nyborg [7] in 1962. They drilled $200 \mu \mathrm{m}$ diameter holes in a sonotrode tip in order to promote cavitation activity at acoustic amplitudes below the threshold of cavitation nucleation. In the experiments bubble growth, surface instabilities and bubbles leaving the holes were observed. Other small amplitude effects were demonstrated by Miller et. al [8] in 1979. In this and in following studies the bubble-mediated biological effects of ultrasound were investigated using thin membranes with micropores of about $4 \mu \mathrm{m}$ in diameter. Aggregation of blood platelets around the cylindrical bubbles was observed which they attributed to radiation pressure and microstreaming.

Clearly, the acoustic response of an entrapped surface bubble shows many similarities with its free floating counterpart. What distinguishes it from a spherical bubble however is not only its stability but also the controllability in size, shape and location, especially with the availability of modern micromachining techniques. Since a stabilized bubble does not need to be nucleated like a free cavitation bubble, its acoustically induced effects such as surface oscillations and microstreaming can be effectuated at much reduced acoustic pressure.

These practical advantages have only recently been translated into some interesting applications. For example, Liu et al. 9] demonstrated rapid mixing by microstreaming in a lab-on-a-chip device using an array of entrapped microbubbles. A stabilized bubble can also be used as a sensitive acoustic sensor as was shown by Rathgen [10]. In this study the acoustically induced displacement of a liquid-gas micromeniscus situated in the tip of an optical fiber was measured using fiber optical interferometry.

The unique and useful properties of stabilized bubbles warrants far more attention than received until now. Theoretical descriptions for the crevice bubble are available from the heterogeneous nucleation theory, however these are based on a static equilibrium of the liquid gas interface and therefore likely to approximate only the low frequency response [5, 11]. Approximate expressions for the resonance frequency and damping terms were derived by Miller \& Nyborg [12] using the reaction force on the liquid-gas interface. To date, the only other investigation was done by Rathgen \& Sugiyama [10]. In this study a solution was found for the small amplitude oscillations from the unsteady Stokes equation. Both models are based on imposing a parabolic liquid gas interface which restricts the response of the system to its fundamental oscillation mode. It is however expected that such a shape might not be representative at higher 
frequencies. For example, let's consider a cylindrical micropit with a circular liquid-gas interface spanning the edge of the pit. Based on this geometry it is reasonable to assume that surface modes may occur which are largely determined by the zeros of the zeroth-order Bessel function, that is $J_{0}(k a)=0$, with $a$ the radius of the cylindrical micropit and $k$ the wavenumber. With $k a=j_{n}$ where $j_{n}$ is the $\mathrm{n}$-th zero corresponding to the $\mathrm{n}$-th mode, the frequency of the capillary wave is estimated using the Kelvin equation [13],

$$
f_{n}=\frac{1}{2 \pi} \sqrt{\frac{\sigma}{\rho}\left(\frac{j_{n}}{a}\right)^{3}} .
$$

In particular, the pits with micrometer-order radii are therefore expected to display surface mode oscillations in the $\mathrm{kHz}$ range frequencies. For example, according to (2.1) a micropit with a radius of $20 \mu \mathrm{m}$ would have its first 3 eigenmodes at $160 \mathrm{kHz}, 560 \mathrm{kHz}$ and $1 \mathrm{MHz}$. This is well within the range of frequencies which are commonly used in many applications.

To investigate the interface and its dynamics in more detail we extend upon the previous work by determining the shape of the liquid gas interface from the boundary conditions, where the contact line remains pinned to the edge of the micropit. In the following we will first consider the mechanical and diffusive stability of an entrapped microbubble under static conditions, after which we will proceed to derive the linear surface oscillation of the system under continuous sinusoidal acoustic driving.

\subsection{A cylindrical micropit bubble}

Our aim is to describe and understand the stability and acoustic response of an entrapped bubble inside a solid object. Since the pits and crevices generally come in many shapes and sizes we restrict ourselves to a mathematically convenient axisymmetric cylindrical micropit with radius $a$ and depth $h$ in an infinite volume of liquid (see Figure 2.1). The liquid-gas interface is assumed to remain pinned at the circular edge of the micropit and its axisymmetric elevation is described with $\eta(r, t)$, or implicitly with

$$
S_{\eta}(r, z, t)=z-\eta(r, t)=0
$$

from which the surface normal can be found using $\mathbf{n}=\frac{\nabla S_{\eta}}{\left|\nabla S_{\eta}\right|}$.

The interface between two media is of crucial importance in any two phase model and its location as a function of time is often taken as a representative description for the system, e.g. $R(t)$ for a spherical bubble. Strictly speaking, 


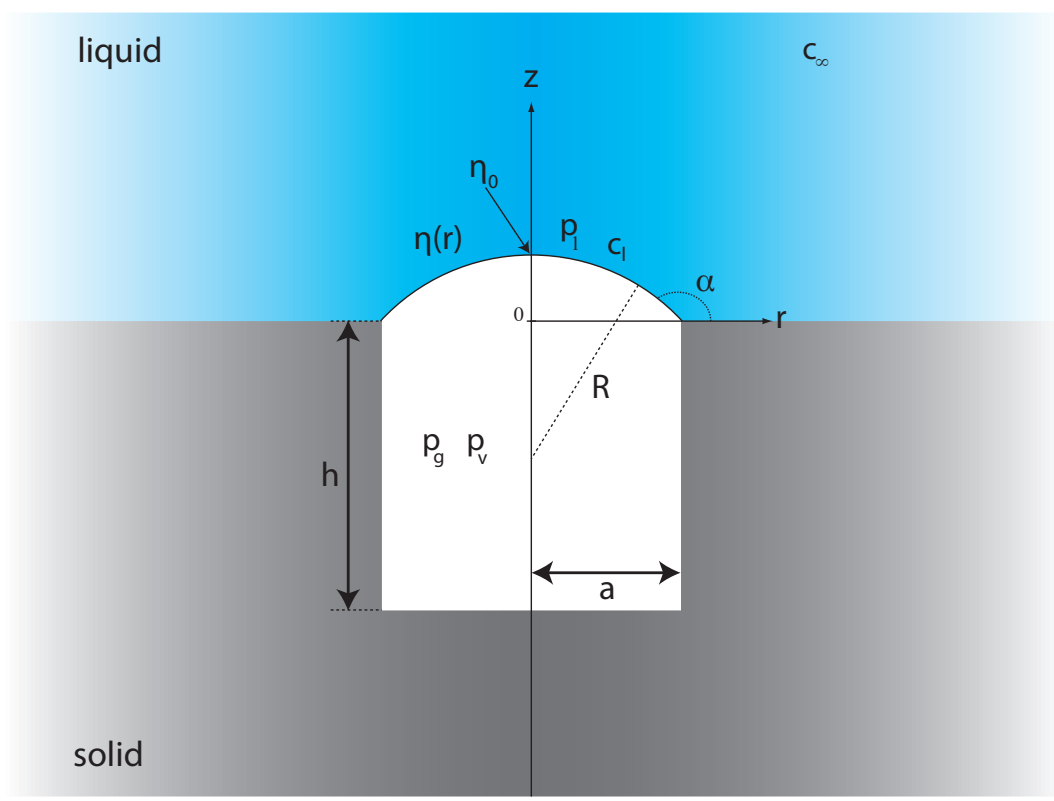

Figure 2.1: Side view of a cylindrical microcavity in which a pocket of gas is stabilized.

the coupling between the two domains is provided through continuity of mass, momentum and energy across the interface. However, since we are interested in the acoustic response, only the continuity of momentum between the two phases is required. When applied to an infinitesimal surface element $d A$ with perimeter $d l$ and surface normal $\mathbf{n}$ this condition reads

$$
\tau_{b} \cdot \mathbf{n} d A=\tau_{l} \cdot \mathbf{n} d A+\sigma \oint \mathbf{m} d l
$$

Because the relative momentum of the liquid and the gas with respect to the bubble wall velocity is assumed negligible, this equation is basically a stress balance between the bubble interior $\left(\tau_{b}\right)$ and the liquid $\left(\tau_{l}\right)$. Here surface tension $\sigma$ is included which is the force per unit length working perpendicular on the perimeter of the surface element but tangential to the interface (i.e. direction $\mathbf{m})$. The stress associated with surface tension can be rewritten as $\sigma \mathbf{m} d l=(\nabla \sigma-\sigma \mathbf{n}(\nabla \cdot \mathbf{n})) d A$ using the Stokes theorem. Assuming that surface tension is constant (hence no Marangoni effects since $\nabla \sigma=0$ ) the stress balance becomes

$$
\left(\tau_{l}-\tau_{b}\right) \cdot \mathbf{n}=\sigma(\nabla \cdot \mathbf{n}) \mathbf{n}
$$


The stress tensors in this equation need to be specified. For an incompressible Newtonian liquid this is given by

$$
\tau_{l}=-p_{l} \mathbf{I}+\mu\left[\nabla \mathbf{u}+(\nabla \mathbf{u})^{T}\right]
$$

where $\mathbf{I}$ is the identity tensor and $p_{l}$ and $\mathbf{u}$ are the liquid pressure and velocity field. Since the viscosity $\mu$ of the content of the bubble interior is much smaller than that of the liquid, the stress tensor inside the bubble is usually considered isotropic, hence

$$
\tau_{b}=-\left(p_{g}+p_{v}\right) \mathbf{I}
$$

where $p_{g}$ and $p_{v}$ are the spatially uniform gas pressure and vapor pressure.

\subsubsection{Stability of the micropit bubble}

It is of interest to investigate the conditions under which a cylindrical micropit bubble is stable in an isothermal stagnant liquid $(\mathbf{u}=0)$ with a dissolved gas concentration of $c_{\infty}$ (see Figure 2.1). Assuming that the contact line is fixed, the shape of the interface is determined by the stress balance as given by (2.4). Since there is no liquid flow, the stress component tangential to the interface is zero. From the normal component of the stress balance the well known Young-Laplace's equation [14] is retrieved:

$$
p_{g}+p_{v}=p_{l}+\sigma \nabla \cdot \mathbf{n}
$$

In general, the exact shape of the static interface can be found by (numerically) solving this equation imposing the contact angle as a boundary condition. However, we are dealing with microscopic length scales much smaller than the capillary length (about $1 \mathrm{~mm}$ in water), therefore the influence of gravity is negligible and the shape of the liquid-gas meniscus is closely approximated by a spherical cap with radius of curvature $R$. As a result, the divergence term in equation (2.7) can be written in its more familiar form $\nabla \cdot \mathbf{n}=2 / R$. Note that this approximation cannot be made a priori for an acoustically driven micromeniscus.

The stability of a liquid-gas interface not only requires a mechanical equilibrium (equation 2.7) but evidently also the net mass and heat flow across the bubble interface should be zero. If this is not the case the bubble either grows or shrinks in response to the imbalance. The gas concentration $c_{l}$ in the liquid immediately adjacent to the liquid-gas interface is given by Henry's Law

$$
c_{l}=K(T) p_{g}
$$


in which $K(T)$ is Henry's constant which depends on the species of gas and solvent and is a function of temperature. When $c_{l}$ is larger than the gas concentration in the bulk of the liquid $\left(c_{\infty}\right)$, diffusion will transport the gas molecules away from the interface causing the crevice bubble to shrink. Similarly, the bubble will grow when $c_{l}$ is smaller than $c_{\infty}$. Hence a diffusive equilibrium can only exist when $c_{l}=c_{\infty}$ and therefore the criterion for diffusive stability is:

$$
p_{g}=\frac{c_{\infty}}{K}
$$

In other words, the bubble is in equilibrium when both equation (2.7) and (2.9) are satisfied for a constant temperature. In order to examine the possible equilibrium states and their stability it is more intuitive to consider the location of the apex $\eta_{0}=\left.\eta\right|_{r=0}$ of the meniscus instead of its radius of curvature $R$. The geometrical relation between both parameters is:

$$
R=\frac{a^{2}+\eta_{0}^{2}}{2 \eta_{0}}
$$

We take $p_{l}$ equal to the ambient pressure $p_{0}$ and the gas concentration at saturation is $c_{s}=K p_{0}$. Using equations (2.7) and (2.10) $p_{g}$ can now be expressed as

$$
p_{g}=p_{0}-p_{v}+\frac{4 \sigma \eta_{0}}{a^{2}+\eta_{0}^{2}}
$$

It is convenient to non-dimensionalize this equation by dividing by $p_{0}$ and introduce the dimensionless surface tension $\beta$ defined as

$$
\beta \equiv \frac{2 \sigma}{a p_{0}}
$$

resulting in

$$
P \equiv \frac{p_{g}}{p_{0}}-1=-\frac{p_{v}}{p_{0}}+\frac{2 \beta\left(\frac{\eta_{0}}{a}\right)}{1+\left(\frac{\eta_{0}}{a}\right)^{2}} .
$$

The graph of $P$ as a function of $\eta_{0} / a$ is the solid blue line shown in Figure (2.2). It is illustrative to compare the micropit bubble with a free spherical bubble having an equilibrium radius $R_{b 0}=a$. From equation (2.7) the nondimensional gas pressure for a bubble of radius $R_{b}$ is found:

$$
P_{b}=-\frac{p_{v}}{p_{0}}+\frac{a \beta}{R_{b}}
$$

the graph of which is the gray line in Figure (2.2) plotted for the non-dimensional bubble radius $\frac{R_{b}}{a}$. Notice that unlike for a spherical bubble, the dimensionless 


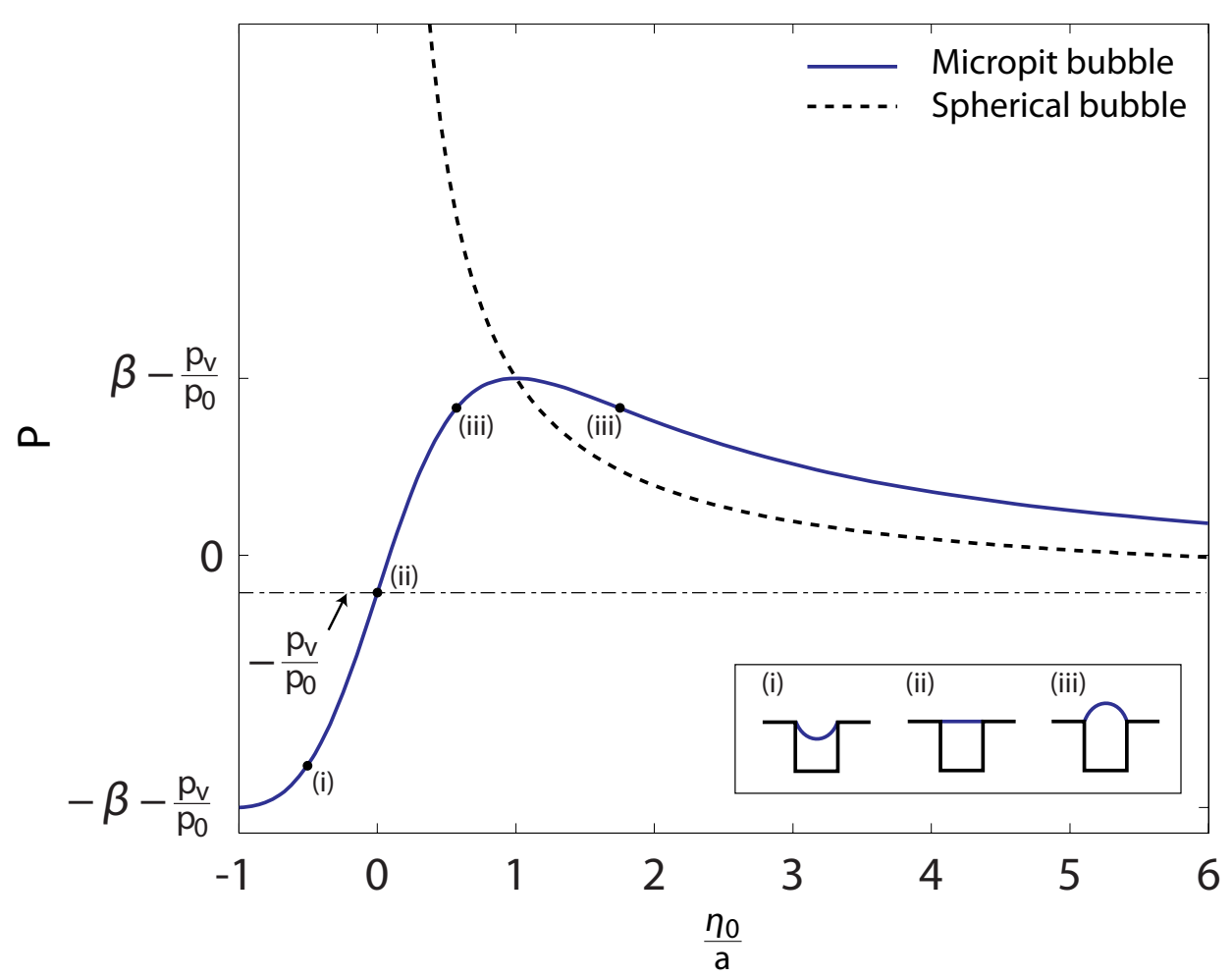

Figure 2.2: Graph of the dimensionless gas pressure in a micropit (equation 2.13) as a function of the position of the apex of the meniscus relative to the substrate surface level. The dashed line is the dimensionless gas pressure in a spherical bubble plotted for $\frac{R_{b}}{a}$, with equilibrium radius $R_{b 0}=a$. The inset schematically shows the interface bend inwards (i), flat (ii) and bend outwards (iii) corresponding to the points marked in the graph.

gas pressure $P$ can be negative for the micropit bubble corresponding to an interface which is bend inwards (case (i)).

The dimensionless form of the condition for diffusive equilibrium (equation 2.9) can now be written as

$$
P=\frac{c_{\infty}}{K p_{0}}-1=\frac{c_{\infty}}{c_{s}}-1 \equiv S
$$

where $S$ is the supersaturation of the gas in the liquid. If $S=-\frac{p_{v}}{p_{0}}$ the interface of the micropit bubble is flat (inset Figure 2.2 (ii)). For larger gas concentrations but with $S<\left(\beta-\frac{p_{v}}{p_{0}}\right)$, the bubble will bulge out of the opening of the crevice $\left(\frac{\eta_{0}}{a}>0\right.$, case (iii)). Here, two equilibrium states exist, either with $\eta_{0}<a$ or 
with $\eta_{0}>a$. However the latter state is unstable which can be understood from the "small-variation-argument" [5]. A slight increase in the bubble size is associated with a decrease in $p_{g}$. Hence $c_{l}=K p_{g}$ will be smaller than $c_{\infty}$ and gas molecules will flow into the bubble thereby increasing $\eta_{0}$ even more. The opposite is of course true for the states where $\eta_{0}<a$. Stated formally, a micropit bubble is in stable equilibrium when

$$
\left\{\begin{array}{l}
\text { (1) } \quad P=S \\
\text { (2) } \frac{d P}{d\left(\eta_{0} / a\right)}>0 .
\end{array}\right.
$$

corresponding to

$$
-\beta-\frac{p_{v}}{p_{0}}<S<\beta-\frac{p_{v}}{p_{0}}
$$

with

$$
-1<\frac{\eta_{0}}{a}<1 .
$$

Note that the actual limits for $\frac{\eta_{0}}{a}$ are determined by the receding contact angle inside the pit and the advancing contact angle on the solid substrate. Applying the criteria of $(2.16)$ to the spherical bubble reveals that although equilibrium states exist when $\frac{p_{v}}{p_{0}}<S<\infty$, these are always unstable because $\frac{d P_{b}}{d\left(R_{b} / a\right)}<0$.

The existence of a maximum value of $P$ as a function of $\frac{\eta_{0}}{a}$ indicates that if the gas saturation $S>\left(\beta-\frac{p_{v}}{p_{0}}\right)$ no equilibrium can exist and the bubble will grow without bounds. This is a condition occurring in most carbonated beverages giving rise to effervescence [15, 16]. The maximum gas concentration for which the micropit bubble can remain stable follows from $(2.13)$ and $(2.15)$ and is

$$
c_{\infty}^{\max }=c_{s}\left(1+\frac{2 \sigma}{a p_{0}}\right) .
$$

The maximum radius $a_{\max }$ of the micropit for which the bubble can remain stable for a certain value of the supersaturation $S$ follows from (2.17):

$$
a \leq a_{\max }=\frac{2 \sigma}{\left|p_{0} S+p_{v}\right|} .
$$

The maximum radius $a_{\max }$ of a micropit in water as a function of the supersaturation $S$ is shown in Figure (2.3). The existence of the singularity at $S=-\frac{p_{v}}{p_{0}}$ indicates that an entrapped cylindrical bubble of any radius with a flat interface is in stable equilibrium. In this state the curvature is infinite and therefore independent of the radius of the micropit. Any deviation from this saturation value considerably limits the radii of the pits which can be stable to microscopic sizes or less. Hence an interesting consequence of equation (2.20) is that it gives an 


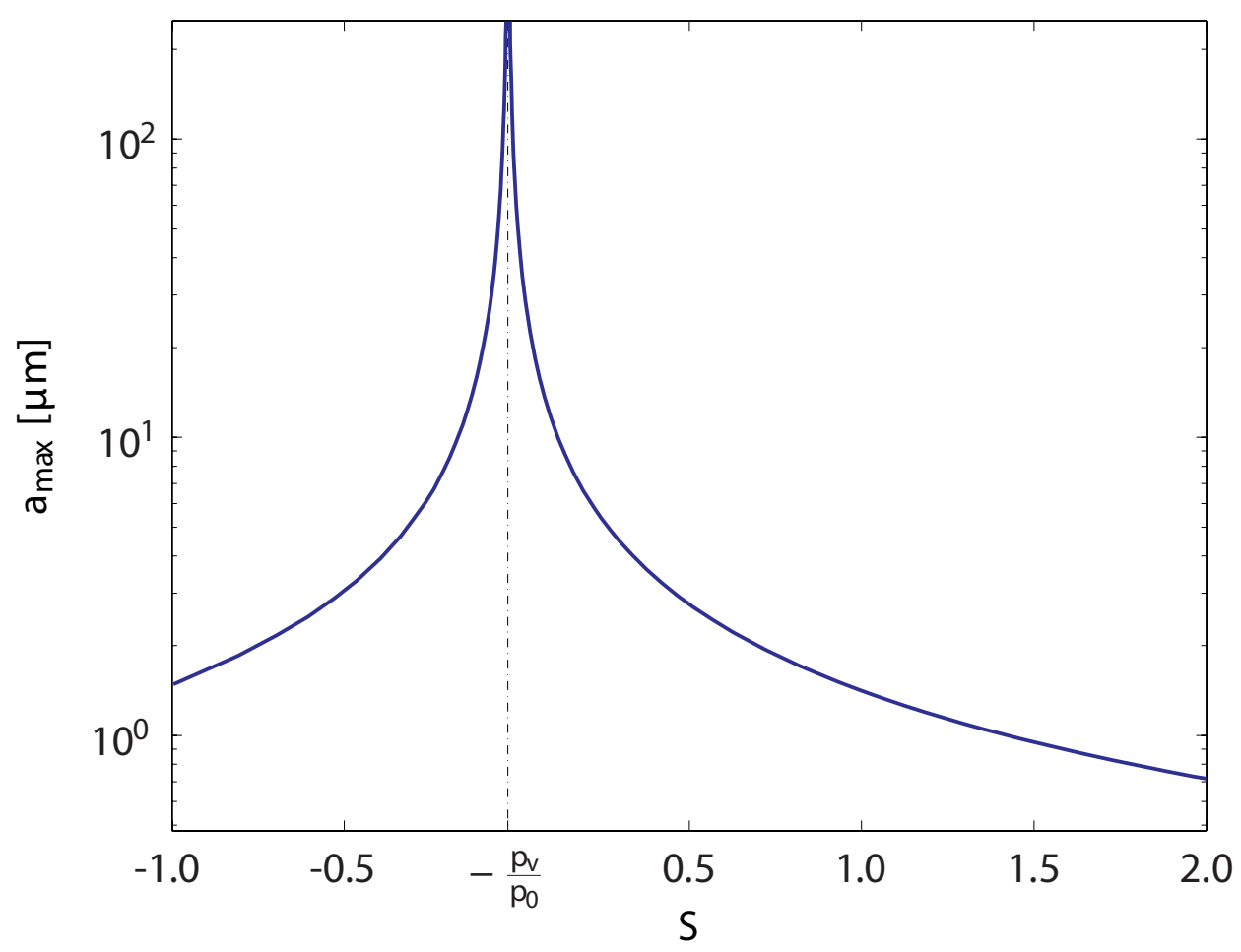

Figure 2.3: Maximum radius of the micropit as a function of supersaturation $\mathrm{S}$ (equation 2.20 for which the micropit bubble can remain stable. Here it is assumed that $h>a$.

upper limit of the size distribution of stable micropit bubbles in a system. Remarkably, it also shows that even completely degassing of a liquid $(S=-1)$ will not necessarily deactivate all the micropits and crevices in the system. It should be noted however that at low gas concentrations the liquid gas interface can bend inwards thereby exceeding the receding contact angle thus causing it to move further down into the pit. Figure 2.3 is an illustration of the exceptional stability of micropit bubbles which supports the likelihood of their existence. 


\subsection{Acoustic response of a micropit bubble}

Similar to a spherical bubble, an entrapped (cylindrical) micropit bubble will respond to an applied acoustic field by expansion and contraction. The system is now 3-dimensional and includes a contactline which makes derivation of a "Rayleigh-Plesset-like" equation for a micropit rather complicated. In addition, the interface cannot be assumed to take a certain shape hence it must be treated as a yet unknown function. As a first approximation, the small amplitude response of the system is investigated. If the velocity scale is estimated to be $\eta_{0} \omega$ with $\eta_{0}$ the amplitude of oscillation and $\omega$ the radial driving frequency of the acoustic field, a comparison between the convective and the local derivative terms in the Navier-Stokes equations results in

$$
\left|\frac{\mathbf{u} \cdot \nabla \mathbf{u}}{\frac{\partial \mathbf{u}}{\partial t}}\right| \approx \frac{\frac{\eta_{0}^{2} \omega^{2}}{a}}{\eta_{0} \omega^{2}}=\frac{\eta_{0}}{a} .
$$

Hence it follows that the convective derivative can be neglected if $\eta_{0} \ll a$. The Navier-Stokes equation then reduces to the incompressible unsteady Stokes equation

$$
\frac{\partial \mathbf{u}}{\partial t}=-\frac{1}{\rho} \nabla p+v \nabla^{2} \mathbf{u} .
$$

In the remainder of this chapter, all equations are non-dimensionalized using

$$
\begin{gathered}
\hat{\mathbf{r}}=a \mathbf{r}, \quad \hat{\mathbf{u}}=\frac{v}{a} \mathbf{u}, \quad \hat{t}=\frac{a^{2}}{v} t, \\
\hat{\omega}=\frac{v}{a^{2}} \omega, \quad \hat{p}=\frac{\rho v^{2}}{a^{2}} p
\end{gathered}
$$

where the hat symbol ${ }^{\wedge}$ indicates dimensional quantities. Assuming a linear time dependence with $e^{i \omega t}$ and applying the axial symmetry, the elevation of the interface and the liquid velocity and pressure are written as

$$
\begin{aligned}
& \eta(r, t)=\tilde{\eta}(r) e^{i \omega t} \\
& \mathbf{u}(r, z, t)=\tilde{\mathbf{u}}(r, z) e^{i \omega t} \\
& p(r, z, t)=p_{0}+\tilde{p}(r, z) e^{i \omega t}+p_{a c} e^{i \omega t}
\end{aligned}
$$

where it is understood that the real part of the right hand side is taken. Also, $p_{a c}$ is the acoustic amplitude of the applied pressure field which is assumed spatially uniform, corresponding to wavelengths much longer than the dimensions of the micropit. The continuity equation and the unsteady Stokes equation now take the form

$$
\begin{aligned}
& \nabla \cdot \tilde{\mathbf{u}}=0 \\
& i \omega \tilde{\mathbf{u}}=-\nabla \tilde{p}+\nabla^{2} \tilde{\mathbf{u}}
\end{aligned}
$$


where $\nabla^{2}$ is the vector Laplacian. These equations need to be solved for $\tilde{\mathbf{u}}$ and $\tilde{p}$ for the appropriate boundary conditions on the gas-liquid and the solid-liquid interface. The general solutions can be found by exploiting the axial symmetry of the system. Because of this symmetry a stream function $\psi(r, z)$ can be defined such that (2.27) is identically satisfied with

$$
\tilde{\mathbf{u}}=\nabla \times\left(\frac{\psi}{r} \mathbf{e}_{\theta}\right) .
$$

where $\mathbf{e}_{\theta}$ is the azimuthal unit vector. The vorticity is then

$$
\boldsymbol{\Omega}=\nabla \times \tilde{\mathbf{u}}=-\nabla^{2}\left(\frac{\psi}{r} \mathbf{e}_{\theta}\right)
$$

with which the unsteady Stokes equation (2.28) becomes

$$
i \omega \Omega=\nabla^{2} \boldsymbol{\Omega}
$$

with $\boldsymbol{\Omega}=\Omega(r, z) \mathbf{e}_{\theta}$. The general solution for $\Omega(r, z)$ is [17]

$$
\Omega(r, z)=\int_{0}^{\infty} G(\lambda) J_{1}(r \lambda) e^{-z \sqrt{\lambda^{2}+i \omega}} d \lambda .
$$

where $G(\lambda)$ is a yet unknown function and it is assumed that $\lim _{z \rightarrow \infty} \tilde{\mathbf{u}}(r, z)=0$ and therefore $\lim _{z \rightarrow \infty} \Omega(r, z)=0$. With this solution, an expression for the stream function can be obtained from (2.30). We write $\psi$ as the sum of the homogeneous solution $\psi_{0}$ and a particular solution $\psi_{p}$. For the first of these $\left(-\nabla^{2}\left(\frac{\psi_{0}}{r} \mathbf{e}_{\theta}\right)=0\right)$ the solution is [17]

$$
\psi_{0}(r, z)=r \int_{0}^{\infty} F(\lambda) J_{1}(r \lambda) e^{-\lambda z} d \lambda
$$

with $F(\lambda)$ a second unknown function, while the particular solution is easily found by inspection from equation (2.30) and (2.31) hence

$$
\frac{\psi_{p}}{r} \mathbf{e}_{\theta}=\frac{i}{\omega} \mathbf{\Omega}
$$

The stream function is therefore

$$
\psi(r, z)=r \int_{0}^{\infty} F(\lambda) J_{1}(r \lambda) e^{-\lambda z} d \lambda+\frac{i r}{\omega} \int_{0}^{\infty} G(\lambda) J_{1}(r \lambda) e^{-z \sqrt{\lambda^{2}+i \omega}} d \lambda .
$$

The velocity components are obtained by substitution in (2.29):

$$
\begin{aligned}
& \tilde{u}_{r}(r, z)=\int_{0}^{\infty} \lambda F(\lambda) J_{1}(r \lambda) e^{-\lambda z} d \lambda+\frac{i}{\omega} \int_{0}^{\infty} \sqrt{\lambda^{2}+i \omega} G(\lambda) J_{1}(r \lambda) e^{-z \sqrt{\lambda^{2}+i \omega}} d \lambda \\
& \tilde{u}_{z}(r, z)=\int_{0}^{\infty} \lambda F(\lambda) J_{0}(r \lambda) e^{-\lambda z} d \lambda+\frac{i}{\omega} \int_{0}^{\infty} \lambda G(\lambda) J_{0}(r \lambda) e^{-z \sqrt{\lambda^{2}+i \omega}} d \lambda
\end{aligned}
$$


Now an expression for the pressure remains to be found. For this purpose, expanding (2.29) and comparison with (2.28) leads to

$$
\nabla \times\left(\frac{\psi_{0}}{r} \mathbf{e}_{\theta}\right)=\frac{i}{\omega} \nabla \tilde{p}
$$

and therefore

$$
\tilde{p}(r, z)=i \omega \int_{0}^{\infty} F(\lambda) J_{0}(r \lambda) e^{-\lambda z} d \lambda
$$

The functions $F(\lambda)$ and $G(\lambda)$ in the expressions for $\tilde{\mathbf{u}}$ and $\tilde{p}$ are determined by the kinematic and dynamic boundary conditions. These will be derived in the following sections.

\subsubsection{The kinematic boundary conditions}

The kinematic boundary condition on $0 \leq r<1$ follows from the invariance of a fluid particle on the interface which is expressed as

$$
\frac{D S_{\eta}}{D t}=\frac{\partial S_{\eta}}{\partial t}+\mathbf{u} \cdot \nabla S_{\eta}=0
$$

where substitution of $(2.2)$ results in

$$
-i \omega \tilde{\eta}-\left.\frac{\partial \tilde{\eta}}{\partial r} \tilde{u}_{r}\right|_{z=\tilde{\eta}}+\left.\tilde{u}_{z}\right|_{z=\tilde{\eta}}=0 .
$$

The velocity components at the interface can be approximated at $z=0$ using a Taylor expansion with

$$
\left.\tilde{u}_{z}\right|_{z=\eta}=\left.\tilde{u}_{z}\right|_{z=0}+\left.\tilde{\eta} \frac{\partial \tilde{u}_{z}}{\partial z}\right|_{z=0}+\ldots
$$

In first approximation all terms of second order and higher are neglected, writing

$$
\left.\tilde{u}_{z}\right|_{z=0}=i \omega \tilde{\eta} \quad \text { on } \quad 0 \leq r<1 .
$$

The kinematic boundary condition on the solid-liquid interface outside the perimeter of the micropit results from its non-permeability stated as

$$
\tilde{u}_{z}=0 \quad \text { on } \quad 1<r<\infty .
$$

In addition, it is assumed that the velocity far from the interface is zero therefore

$$
\tilde{\mathbf{u}}=0 \quad \text { for } z \rightarrow \infty,
$$

a condition which was already fulfilled in the derivation of (2.32) and (2.33). 


\subsubsection{The dynamic boundary conditions}

The dynamic boundary conditions result from the balance of stress on the liquid gas interface given by (2.4). First the stress in the normal direction is considered. Non-dimensionalizing and substitution of (2.5) and (2.6) into (2.4) results in

$$
p_{b}-p_{l}+\mathbf{n} \cdot\left(\nabla \mathbf{u}+\nabla \mathbf{u}^{T}\right) \cdot \mathbf{n}=\mathrm{Ca}^{-1} \nabla \cdot \mathbf{n} \quad \text { on } \quad 0 \leq r<1
$$

where the $\mathrm{Ca}$ is the Capillary number given by

$$
\mathrm{Ca}=\frac{\mu U}{\sigma}=\frac{\rho v^{2}}{\sigma a}
$$

and where $U=\frac{v}{a}$. The separate terms in (2.46) need further specification. First of all the surface normal is

$$
\mathbf{n}=\frac{\nabla S_{\eta}}{\left|\nabla S_{\eta}\right|}=\frac{-\tilde{\eta}^{\prime} \mathbf{e}_{r}+\mathbf{e}_{z}}{\sqrt{\left(\tilde{\eta}^{\prime}\right)^{2}+1}} \approx-\tilde{\eta}^{\prime} \mathbf{e}_{r}+\mathbf{e}_{z}
$$

where $\mathbf{e}_{r}$ and $\mathbf{e}_{z}$ are the unit vectors. The prime denotes the derivative over $r$. So we find for the divergence term

$$
\nabla \cdot \mathbf{n}=-\frac{\left(\tilde{\eta}^{\prime}\right)^{3}+\tilde{\eta}^{\prime}+r \tilde{\eta}^{\prime \prime}}{r\left(\left(\tilde{\eta}^{\prime}\right)^{2}+1\right)^{\frac{3}{2}}} \approx-\left(\tilde{\eta}^{\prime \prime}+\frac{1}{r} \tilde{\eta}^{\prime}\right) .
$$

Similarly, the viscous stress normal to the interface evaluates to

$$
\left.\mathbf{n} \cdot\left(\nabla \tilde{\mathbf{u}}+\nabla \tilde{\mathbf{u}}^{T}\right) \cdot \mathbf{n} \approx 2 \frac{\partial \tilde{u}_{z}}{\partial z}\right|_{z=0} .
$$

The gas inside the pit is assumed to be homogeneous and to follow the perfect gas law, hence

$$
\hat{p}_{g}=\hat{p}_{0}\left(\frac{\hat{V}_{0}}{\hat{V}}\right)^{\kappa}
$$

with $\kappa$ the polytropic exponent. The bubble volume is given by the volume of the cylindrical micropit plus the part of the volume of the bubble protruding above (or below) the surface level:

$$
\hat{V}=\pi a^{2} h+\pi a^{3} \int_{0}^{\tilde{\eta}(0)} r^{2} d z=\hat{V}_{0}+\pi a^{3} \int_{1}^{0} r^{2} \frac{\partial \tilde{\eta}}{\partial r} d r
$$

Defining

$$
H(\tilde{\eta}) \equiv \int_{1}^{0} r^{2} \frac{\partial \tilde{\eta}}{\partial r} d r=2 \int_{0}^{1} r \tilde{\eta} d r
$$


results in

$$
\hat{V}=\hat{V}_{0}(1+\gamma H(\tilde{\eta}))
$$

with $\gamma=\frac{a}{h}$ the aspect ratio of the micropit. Substitution of this equation into (2.51) and linearization near $\gamma H=0$ (hence the interface is assumed flat when $\left.p_{g}=p_{0}\right)$ gives

$$
p_{g}=p_{0}(1-\kappa \gamma H)
$$

And finally with $p_{l}=\left.p(r, z, t)\right|_{z=0}$ equation (2.46) is written as

$$
-\alpha H-\left.\tilde{p}\right|_{z=0}-p_{a c}+\left.2 \frac{\partial \tilde{u}_{z}}{\partial z}\right|_{z=0}=-\mathrm{Ca}^{-1}\left(\tilde{\eta}^{\prime \prime}+\frac{1}{r} \tilde{\eta}^{\prime}\right)
$$

where $\alpha$ is a non-dimensional quantity defined as

$$
\alpha=\frac{\hat{p}_{0} \kappa \gamma a^{2}}{\rho v^{2}} .
$$

Note here that $\hat{p}_{0}$ is dimensional. After the velocity and pressure field have been derived, equation (2.56) will be used to find an expression for the interface $\tilde{\eta}(r)$. However, also the stress tangential to the interface needs to be taken into account. From (2.4) it follows that

$$
\mathbf{n} \cdot\left(\nabla \mathbf{u}+\nabla \mathbf{u}^{T}\right) \cdot \mathbf{m}=\mathbf{n} \cdot \tau_{b} \cdot \mathbf{m} \quad 0<r<1
$$

while a stress balance on the solid-liquid interface $\left(\tau_{w}\right)$ results in

$$
\mathbf{n} \cdot\left(\nabla \mathbf{u}+\nabla \mathbf{u}^{T}\right) \cdot \mathbf{m}=\mathbf{n} \cdot \tau_{w} \cdot \mathbf{m} \quad r>1
$$

where $\mathbf{m}$ is again the unit vector tangential to the interface. Since the viscosity of the bubble content is much smaller than that of the liquid, it follows that $\mathbf{n} \cdot \tau_{b} \cdot \mathbf{m} \approx 0$ and the boundary condition is basically the "free-slip condition". However equations (2.58) and (2.59) then constitute a mixed boundary condition which due to its complexity is not pursued here. Instead, it is assumed that the interior can support stress which can only be realized if the tangential velocity is zero everywhere on $z=0$ hence,

$$
\left.\tilde{u}_{r}\right|_{z=0}=0 \quad \text { on } \quad 0<r<\infty
$$

which is the well known "no-slip boundary condition". This assumption may be justified in the presence of contaminants such as surfactants which result in an effective shear stress [18]. 
To summarize, the boundary conditions on the liquid-gas interface $(0 \leq r<1)$ are:

$$
\begin{aligned}
& \left.\tilde{u}_{z}\right|_{z=0}=i \omega \tilde{\eta} \\
& \left.\tilde{u}_{r}\right|_{z=0}=0 \\
& -\alpha H-\left.\tilde{p}\right|_{z=0}-p_{a c}+\left.2 \frac{\partial \tilde{u}_{z}}{\partial z}\right|_{z=0}=-\mathrm{Ca}^{-1}\left(\tilde{\eta}^{\prime \prime}+\frac{1}{r} \tilde{\eta}^{\prime}\right)
\end{aligned}
$$

and for the solid-liquid interface $(1<r<\infty)$

$$
\begin{aligned}
& \left.\tilde{u}_{z}\right|_{z=0}=0 \\
& \left.\tilde{u}_{r}\right|_{z=0}=0 .
\end{aligned}
$$

\subsection{Solution}

An interesting side-effect of the system of equations as presented until now is that the potential flow solution can be obtained quite easily. If the flow is irrotational then the vorticity is zero and therefore from (2.32) it follows that $G(\lambda)=0$. This solution will not be further investigated however since for our problem viscous contributions are of interest.

First we consider the vertical kinematic boundary condition on the liquid-gas interface. From (2.37) and (2.61) it follows that

$$
\left.\tilde{u}_{z}\right|_{z=0}=\int_{0}^{\infty} \lambda\left(F(\lambda)+\frac{i}{\omega} G(\lambda)\right) J_{0}(r \lambda) d \lambda=i \omega \tilde{\eta}(r) .
$$

Applying the Hankel transform [19] and invoking (2.64) on the liquid-solid boundary results in

$$
F(\lambda)+\frac{i}{\omega} G(\lambda)=i \omega V(\lambda)
$$

with

$$
V(\lambda)=\int_{0}^{1} \tilde{\eta}(r) r J_{0}(r \lambda) d r
$$

From conditions (2.62) and (2.65) and substitution of (2.36) it follows that

$$
\left.\tilde{u}_{r}\right|_{z=0}=\int_{0}^{\infty}\left(\lambda F(\lambda)+\frac{i}{\omega} \sqrt{\lambda^{2}+i \omega} G(\lambda)\right) J_{1}(\lambda r) d \lambda=0
$$


therefore with equation (2.67) the following expressions for $F(\lambda)$ and $G(\lambda)$ are found:

$$
\begin{aligned}
& F(\lambda)=V(\lambda)\left(\lambda^{2}+i \omega+\lambda \sqrt{\lambda^{2}+i \omega}\right) \\
& G(\lambda)=i \omega V(\lambda)\left(\lambda^{2}+\lambda \sqrt{\lambda^{2}+i \omega}\right)
\end{aligned}
$$

Notice that $F(\lambda)=i \omega V(\lambda)$ relates to the irrotational part of the flow field. With these equations the velocity and pressure field in the liquid are defined, depending on $\tilde{\eta}$ through $V(\lambda)$. In order to determine $\tilde{\eta}(r)$ equations (2.70) and $(2.71)$ are substituted in the dynamic boundary equation $(2.56)$. For $\left.\tilde{p}\right|_{z=0}$ using (2.39) it follows that

$$
\left.\tilde{p}\right|_{z=0}=i \omega \int_{0}^{\infty} V(\lambda)\left(i \omega+\lambda^{2}+\lambda \sqrt{\lambda^{2}+i \omega}\right) J_{0}(r \lambda) d \lambda .
$$

The normal viscous stress is canceled since

$$
\left.\frac{\partial \tilde{u}_{z}}{\partial z}\right|_{z=0}=-\int_{0}^{\infty}\left(F(\lambda) \lambda^{2}+\frac{i}{\omega} G(\lambda) \lambda \sqrt{\lambda^{2}+i \omega}\right) J_{0}(r \lambda) d \lambda=0 .
$$

Hence the dynamic boundary equation (2.56) becomes

$$
\begin{gathered}
-\alpha H-p_{a c}-i \omega \int_{0}^{\infty} V(\lambda)\left(\lambda^{2}+i \omega+\lambda \sqrt{\lambda^{2}+i \omega}\right) J_{0}(r \lambda) d \lambda \\
=-\mathrm{Ca}^{-1}\left(\tilde{\eta}^{\prime \prime}+\frac{1}{r} \tilde{\eta}^{\prime}\right) .
\end{gathered}
$$

A solution for this integro-differential equation for $\tilde{\eta}$ is not readily available. However, considering the axial symmetry it is convenient to expand $\tilde{\eta}(r)$ into a Fourier-Bessel series.

\subsubsection{Solution with Fourier-Bessel series}

The expansion reads

$$
\tilde{\eta}(r)=\sum_{k=1}^{\infty} c_{k} J_{0}\left(j_{k} r\right)
$$

where the $j_{k}$ 's are the zeros of $J_{0}$ and the $c_{k}$ coefficients are to be determined. The expansion of $V(\lambda)$ is given by

$$
V(\lambda)=\sum_{k=1}^{\infty} c_{k} \frac{j_{k} J_{1}\left(j_{k}\right) J_{0}(\lambda)}{j_{k}^{2}-\lambda^{2}}
$$

and with $H=2 V(0)$

$$
H=2 \sum_{k=1}^{\infty} c_{k} \frac{J_{1}\left(j_{k}\right)}{j_{k}}
$$


Also

$$
-\left(\tilde{\eta}^{\prime \prime}+\frac{1}{r} \tilde{\eta}^{\prime}\right)=\sum_{k=1}^{\infty} c_{k} j_{k}^{2} J_{0}\left(j_{k} r\right) .
$$

Now equation 2.74 is written as

$$
\begin{gathered}
-i \omega \sum_{k=1}^{\infty} c_{k} \int_{0}^{\infty} \frac{j_{k} J_{1}\left(j_{k}\right) J_{0}(\lambda)}{j_{k}^{2}-\lambda^{2}}\left(\lambda^{2}+i \omega+\lambda \sqrt{\lambda^{2}+i \omega}\right) J_{0}(r \lambda) d \lambda \\
=p_{a c}+2 \alpha \sum_{k=1}^{\infty} c_{k} \frac{J_{1}\left(j_{k}\right)}{j_{k}}+\mathrm{Ca}^{-1} \sum_{k=1}^{\infty} c_{k} j_{k}^{2} J_{0}\left(j_{k} r\right) .
\end{gathered}
$$

from which the coefficients $c_{k}$ need to be found. Here we make use of the orthogonality of the Bessel functions. Hence multiplying by $r J_{0}\left(j_{n} r\right)$ and integrating over $r$ from 0 to 1 results in

$$
\begin{array}{r}
-i \omega \sum_{k=1}^{\infty} c_{k} j_{k} j_{n} J_{1}\left(j_{k}\right) J_{1}\left(j_{n}\right) \int_{0}^{\infty} \frac{J_{0}^{2}(\lambda)}{\left(j_{k}^{2}-\lambda^{2}\right)\left(j_{n}^{2}-\lambda^{2}\right)}\left(\lambda^{2}+i \omega+\lambda \sqrt{\lambda^{2}+i \omega}\right) d \lambda \\
=p_{a c} \frac{J_{1}\left(j_{n}\right)}{j_{n}}+2 \alpha \sum_{k=1}^{\infty} \frac{c_{k}}{j_{k} j_{n}} J_{1}\left(j_{k}\right) J_{1}\left(j_{n}\right)+\frac{1}{2} \mathrm{Ca}^{-1} c_{n} j_{n}^{2} J_{1}^{2}\left(j_{n}\right) .
\end{array}
$$

This equation is a matrix equation for $c_{k}(\omega)$. The equation is not trivial to solve however in particular due to the integral for which there is no explicit solution. Rewriting the equation in matrix form results in

$$
\mathbf{A}(\omega) \cdot \mathbf{c}=p_{a c} \mathbf{a}+\left(\alpha \mathbf{B}+\mathrm{Ca}^{-1} \mathbf{C}\right) \cdot \mathbf{c} .
$$

where $\mathbf{c}=c_{k} \mathbf{e}_{k}$ and the vector $\mathbf{a}$ and the matrices $\mathbf{A}, \mathbf{B}$ and $\mathbf{C}$ correspond to their respective appearances in equation (2.80).

From (2.81) the relation between the complex amplitude $\mathbf{c}$ and the driving amplitude is

$$
\mathbf{c}=p_{a c}\left(\mathbf{A}(\omega)-\alpha \mathbf{B}-\mathrm{Ca}^{-1} \mathbf{C}\right)^{-1} \cdot \mathbf{a}
$$

with which the coefficients $c_{k}$ 's can be found as a function of $\omega$. The integral in $\mathbf{A}$ is evaluated numerically using the Gauss-Kronod quadrature method as implemented in Matlab R2009b\& [20]. This method accurately calculates the integral solution with oscillatory integrands and infinite intervals to any required precision (here set to 6 digits). The matrices in (2.82) are of infinite dimension which renders them unmanageable. The summations are therefore truncated resulting in $N \times N$ matrices. It was found that $\left|c_{k}\right|$ converges rapidly in all cases and taking $N=30$ is sufficiently accurate. 


\subsection{Results}

\subsubsection{Effective mass and damping}

Similar to a spherical bubble, the entrapped cylindrical bubble can be considered a harmonic oscillator with an effective mass, stiffness and damping, all of which depend on frequency. The effective mass $m$ and the damping $\beta$ are contained within the pressure exerted by the liquid on the interface. Hence if we consider the radiation impedance [12, 21]

$$
Z=\frac{\left\langle\tilde{p}_{z=0}\right\rangle}{i \omega\langle\tilde{\eta}\rangle}
$$

with $\langle\ldots\rangle$ the surface average, we find for $m$ and $\beta$

$$
\begin{aligned}
m & =\frac{1}{\omega} \mathfrak{R}\{Z\} \\
\beta & =\frac{1}{2 m} \mathfrak{I}\{Z\} .
\end{aligned}
$$

The effective stiffness $s$ results from the combination of the compressibility of the bubble interior and the surface tension. From (2.74) this is

$$
s=\alpha+\frac{\left\langle\tilde{\eta}^{\prime \prime}+\frac{1}{r} \tilde{\eta}^{\prime}\right\rangle}{\operatorname{Ca}\langle\tilde{\eta}\rangle} .
$$

Hence the stiffness is increasing with radius through $\alpha(a) \propto a^{2}$ and $\mathrm{Ca}^{-1} \propto a$. In Figure 2.4 the effective mass $\mathrm{m}_{k}(\omega)$ and damping $\beta_{k}(\omega)$ for the first 4 summation terms of the expansion (2.75) are shown as a function of the dimensionless frequency $\omega$. For $\omega>1$ the effective mass decreases from its low frequency asymptote to a constant value at higher frequencies and is smaller for the higher order components. This can be understood from the mass contained in the displaced volume by the different surface shapes which scales with $J_{1}\left(j_{k}\right) / j_{k}$. The effective damping on the other hand increases for $\omega>1$ according to $\beta_{k} \propto \omega^{\frac{1}{2}}$, corresponding to increased energy dissipation in the oscillatory boundary layer with increasing frequency. Here the shape of the interface is of little importance.

\subsubsection{Response of the micromeniscus}

Since all parameters of the harmonic oscillator depend on frequency and surface shape, defining a single resonance frequency is not trivial. We therefore consider the response of the micromeniscus for different frequencies, radii and depth at constant driving amplitude. The physical parameters were set to those of 
(a)

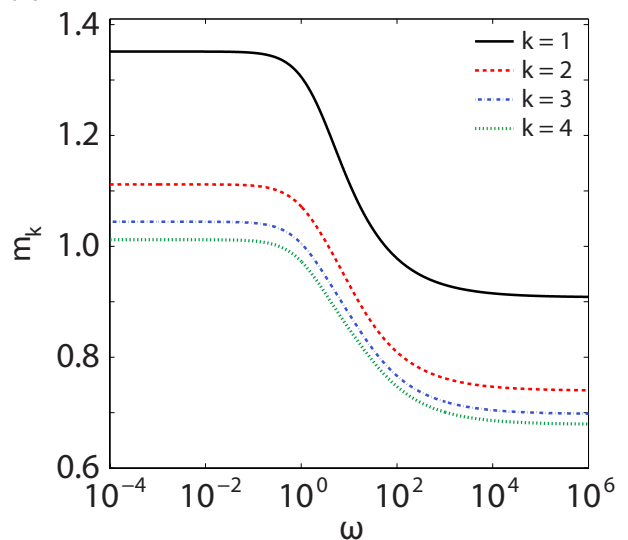

(b)

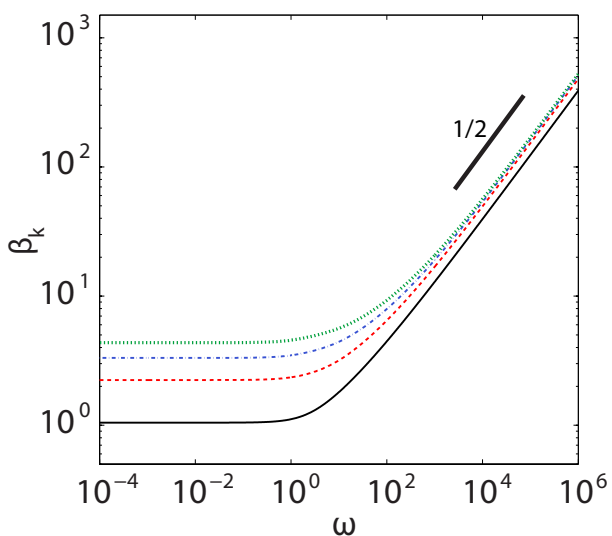

Figure 2.4: The (dimensionless) effective mass $m_{k}(\omega)$ (a) and damping $\beta_{k}(\omega)$ (b) for the first 4 Bessel function components of the interface $(k=1,2,3$ and 4$)$.

water in ambient conditions $\left(\rho=1.0 \cdot 10^{3} \mathrm{~kg} / \mathrm{m}^{3}, \sigma=7.3 \cdot 10^{-2} \mathrm{~N} / \mathrm{m}, v=\right.$ $\left.1.0 \cdot 10^{-6} \mathrm{~m}^{2} / \mathrm{s}, p_{0}=1.0 \cdot 10^{5} \mathrm{~Pa}\right)$. The isotropic coefficient is set at $\kappa=1.2$ in between isothermal and adiabatic conditions. The result for a micropit with a radius of $15 \mu \mathrm{m}$ and equal depth is shown in Figure 2.5. Here the absolute value of the amplitude $\tilde{\eta}$ at $r=0$ is taken as a measure for the amplitude of oscillation. In this case the largest response is found at a frequency of $121 \mathrm{kHz}$, while a second and third maximum arise for $241 \mathrm{kHz}$ and $491 \mathrm{kHz}$. Higher order maxima are also present however these are exceedingly small due to viscous damping. As illustrated in Figure 2.5(b) and (c) the corresponding surface shapes at these maxima have the largest contribution of the first, second and third Bessel component respectively. The neighboring components become increasingly important for higher frequencies.

To explore the effect of the radius $a$ on the oscillation of the meniscus, its response was calculated for values between $1 \mu \mathrm{m}$ and $400 \mu \mathrm{m}$ with $\gamma=\frac{a}{h}=1$. This range corresponds to the radii for which a micropit bubble can be in stable equilibrium in the absence of ultrasound (see section 2.2). The result is presented in Figure 2.6(a) in the radius-frequency domain. In this figure the coloring is in logarithmic scale where bright coincides with maximum response and darker regions indicate small response. The line structure corresponds to the peaks of maximum response which are relatively narrow and well separated for the smaller radii. For larger radii and frequencies increased mass and damping result in much reduced response of the meniscus.

The damped resonance peaks scale with the radius as $a^{-\frac{3}{2}}$ similar to the 

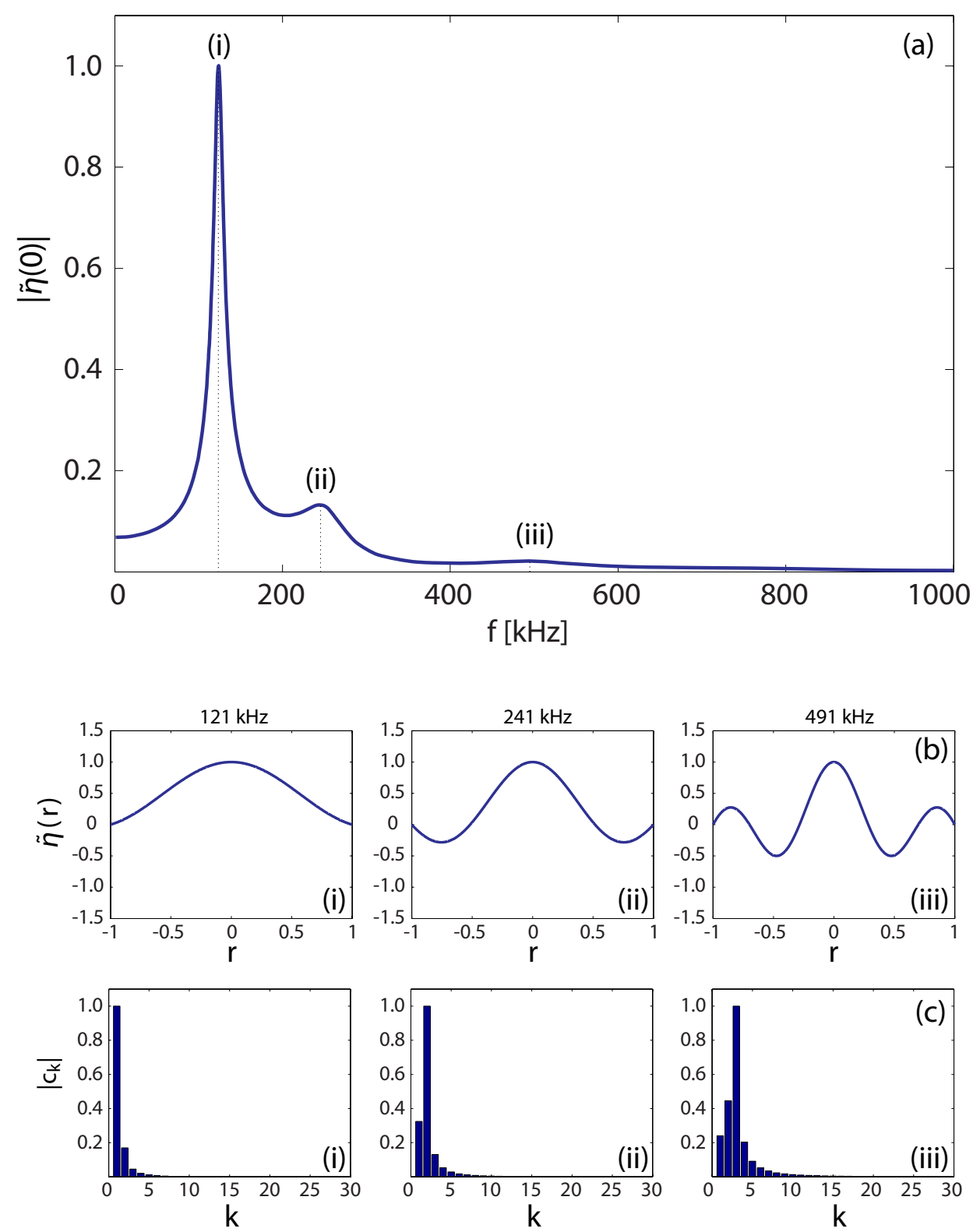

Figure 2.5: (a) Normalized frequency response of a micropit bubble with $a=15 \mu \mathrm{m}$ and $h=15 \mu \mathrm{m}$. For the first 3 maxima (i), (ii) and (iii) the reconstructed interface shapes (b) and the corresponding coefficients $\left|c_{k}\right|$ (c) are shown. 

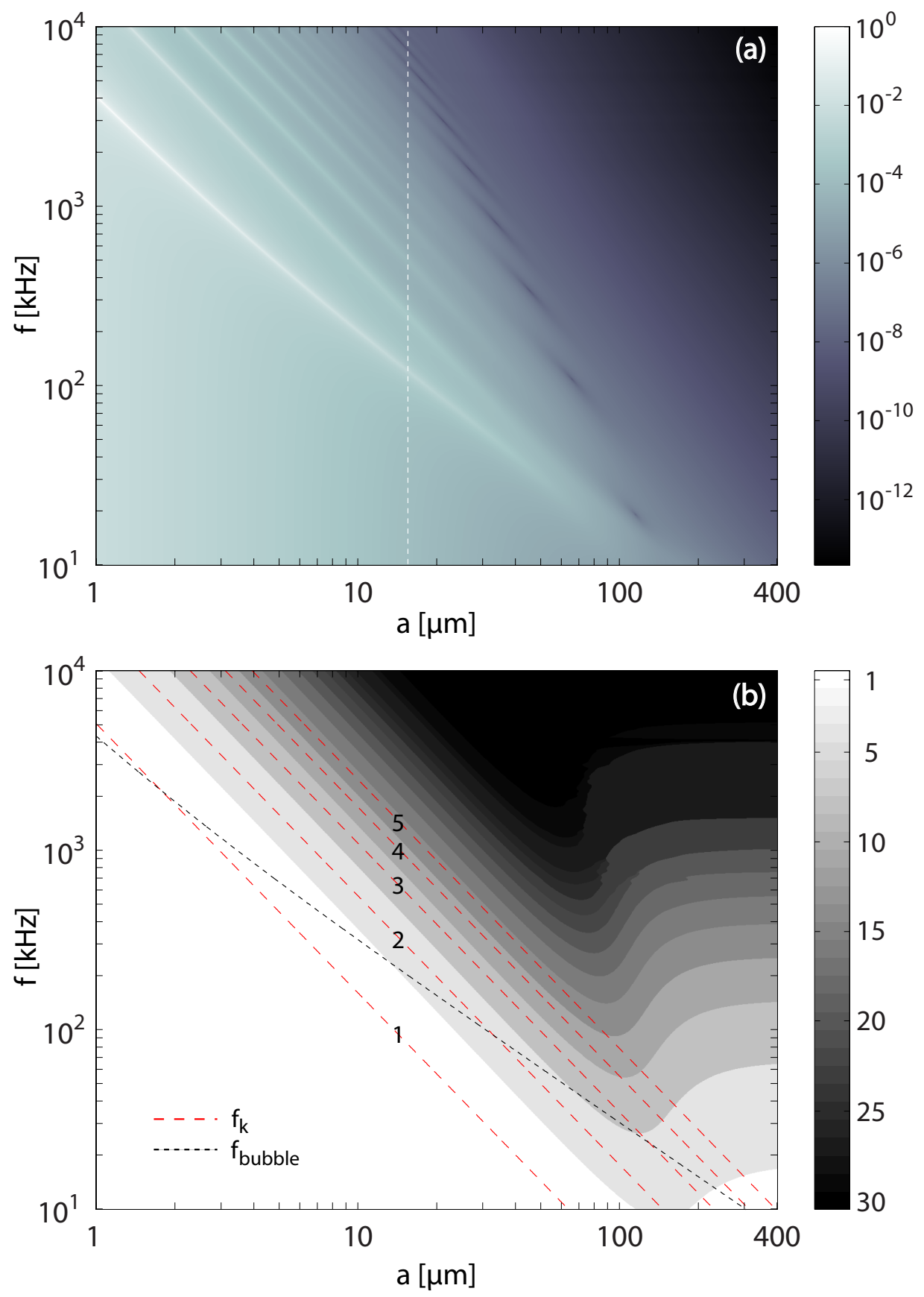

Figure 2.6: (a) The response (logarithmic color scheme) of a cylindrical micropit bubble given by $\left|\hat{\eta}_{0}\right|$ with $a=h$ to sinusoidal driving. The white dashed line at $a=15 \mu \mathrm{m}$ corresponds to the graph shown in Figure 2.5. (b) The regions for the dominant surface components $J_{0}\left(j_{k} r\right)$, together with approximate oscillation frequencies $f_{k}$ of the first 5 surface modes as given by (2.1). Here white is for $k=1$, the neighboring gray band for $k=2$ and so on. 


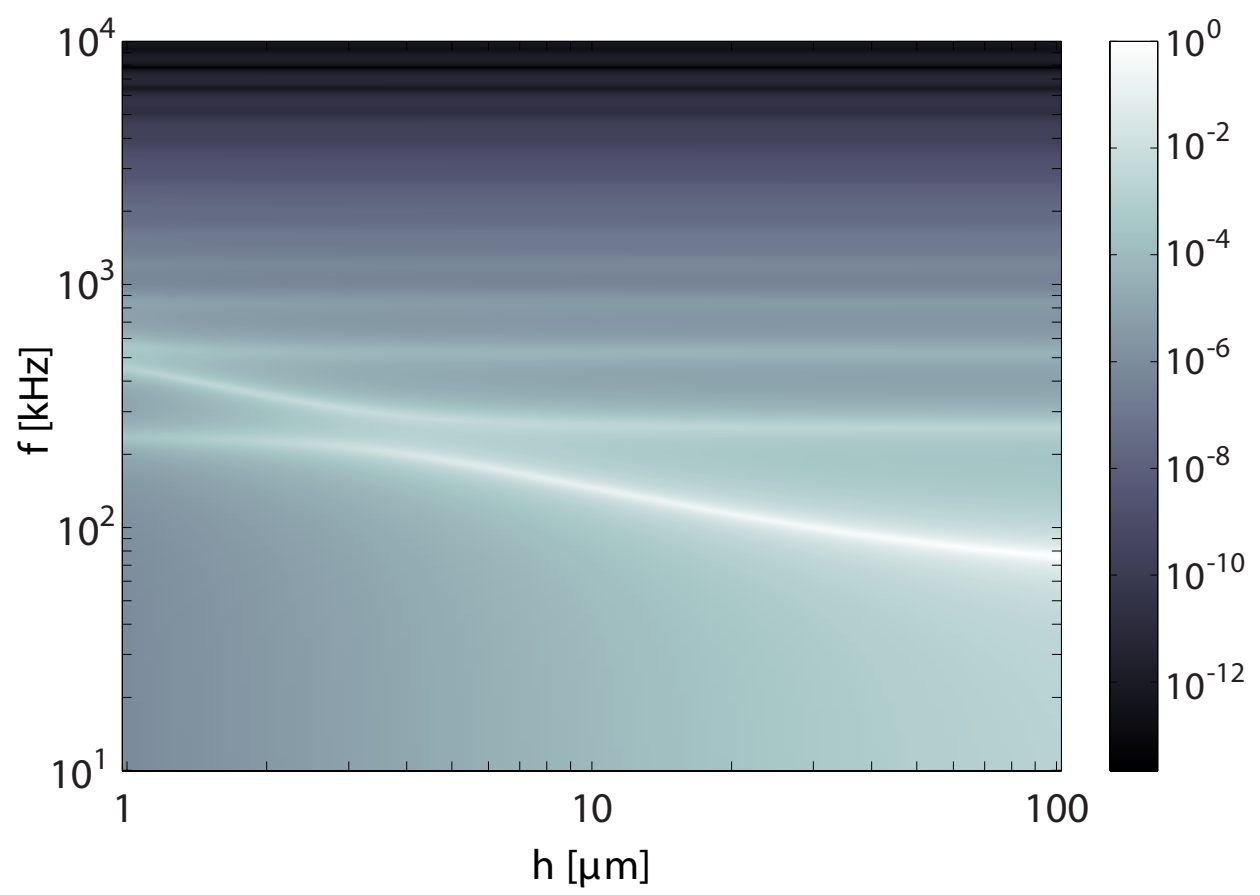

Figure 2.7: Dependence on depth of the response $\left|\hat{\eta}_{0}\right|$ of a $15 \mu \mathrm{m}$ radius micropit.

estimate of equation (2.1). The first resonance peak however deviates from this trend for larger radii. This can be understood from the stiffness of the system (2.86). The contribution by surface tension has a linear dependence on the radius (through $\mathrm{Ca}$ ) while the part due to compressibility of the gas inside the micropit scales with $a^{2}$ (through $\alpha$ ). For small radii the oscillation is dominated by surface tension and the depth of the micropit is of little importance. However from approximately $a=10 \mu \mathrm{m}$ on, the stiffness contributed by the gas prevails over the part contributed by surface tension and $f \propto a^{-1}$ but only for the first resonance peak. The higher resonances contain contributions by higher order surface modes (see Figure 2.6(b)) thereby leading to increased stiffness. Such a dependence on the depth $h$ is illustrated in Figure 2.7.

In Figure 2.6(b) the phase diagram for the dominant surface modes of $\tilde{\eta}$ is shown. The white area indicates the region where the first mode is largest $(k=1)$, while the neighboring gray areas indicate the consecutive higher order modes ( $k=2, k=3$ and so on). For comparison also frequency dependence of the first 5 surface modes as given by (2.1) are shown, together with the linear resonance of a spherical bubble (equation (1.2)) where the micropit radius 
is taken as equal to the bubble radius. In particular in the surface tension dominated region (smaller radii) the micropit bubble and the spherical bubble show similar behavior.

Interestingly the estimate of the modal frequencies lay centered in the regions of the corresponding dominant surface modes. For radii larger than approximately $100 \mu \mathrm{m}$ however the modal frequencies first increase and then flatten out. In this region the oscillation is controlled by damping and mass while the stiffness becomes negligible.

\subsection{Discussion and Conclusion}

In this chapter the static equilibrium and dynamic response of a bubble entrapped in a cylindrical cavity were discussed. This system may be considered a model for the crevice bubble from which cavitation activity can be initiated. Such a bubble is stable against dissolution, in particular for cavities with radii measured in micrometers or less (see Figure 2.3). This range coincides with the micropit bubbles which are likely to display surface mode oscillations as suggested by the linear oscillation model presented here. The presence of these modes were mentioned by Rathgen \& Sugiyama [10] who were "beginning to see the modes of a micrometer sized liquid drum" in their experiments. Also in the experiments presented in chapter 5, ring structures are observed which might be related to these modes. Substantial experimental evidence is however difficult to obtain since the geometry and low reflectivity of the liquid gas interface hampers detailed investigation. Advanced imaging techniques or the use of a vibrometer may provide a solution for this challenge.

The linear model presented in this chapter corresponds to low acoustic amplitude driving. The surface modes however may become unstable for increased amplitudes due to parametric instabilities, similar to behavior known from spherical bubbles [22]. Extension of the current model to include the nonlinear temporal dependence of the mode amplitudes $c_{k}$ is therefore desirable. As will be shown in chapter 5 , destabilization of the micromeniscus results in bubble pinch-off. Further study is required to investigate its relation to the surface ripples. Also the effect of the free-slip boundary condition on the liquid gas interface should be addressed as well as possible non-axisymmetrical vibration modes. Due to the assumption of a no-slip boundary condition the damping is likely to be slightly overestimated. Further refinement of the model can be achieved by including thermal and acoustic dissipation effects. 


\section{Bibliography}

[1] J. Y. Ye, G. Chang, T. B. Norris, C. Tse, M. J. Zohdy, K. W. Hollman, M. O'Donnell, and Jr. J. R. Baker. Trapping cavitation bubbles with a self-focused laser beam. Opt. Lett., 29(18):2136-2138, 2004.

[2] D. F. Gaitan, L. A. Crum, C. C. Church, and R. A. Roy. Sonoluminescence and bubble dynamics for a single, stable, cavitation bubble. J. Acoust. Soc. Am., 91(6):3166-3183, 1992.

[3] M. P. Brenner, S. Hilgenfeldt, and D. Lohse. Single-bubble sonoluminescence. Rev. Mod. Phys., 74(2):425-484, 2002.

[4] E. N. Harvey, D. K. Barnes, W. D. McElroy, A. H. Whiteley, D. C. Pease, and K. W. Cooper. Bubble formation in animals. i. physical factors. J. Cell. Comp. Physiol., 24(1):1-22, 1944.

[5] A. A. Atchley and A. Prosperetti. The crevice model of bubble nucleation. J. Acoust. Soc. Am., 86(3):1065-1084, 1989.

[6] B. M. Borkent, S. Gekle, A. Prosperetti, and D. Lohse. Nucleation threshold and deactivation mechanisms of nanoscopic cavitation nuclei. Physics of Fluids, 21(10):102003, 2009.

[7] D. E. Hughes and W. L. Nyborg. Cell disruption by ultrasound. Science, 138(3537):pp. 108-114, 1962.

[8] D. L. Miller, W. L. Nyborg, and C. C. Whitcomb. Platelet aggregation induced by ultrasound under specialized conditions in vitro. Science, 205 (4405):pp. 505-507, 1979.

[9] R. H. Liu, J. Yang, M. Z. Pindera, M. Athavale, and P. Grodzinski. Bubbleinduced acoustic micromixing. Lab on a Chip, 2:151-157, 2002.

[10] H. Rathgen. Superhydrophobic Surfaces: from Fluid mechanics to Optics. PhD thesis, University of Twente, The Netherlands, 2008.

[11] M. A. Chappell and S. J. Payne. The effect of cavity geometry on the nucleation of bubbles from cavities. J. Acoust. Soc. Am., 121(2):853-862, 2007.

[12] D. L. Miller and W. L. Nyborg. Theoretical investigation of the response of gas-filled micropores and cavitation nuclei to ultrasound. J. Acoust. Soc. Am., 73(5):1537-1544, 1983. 
[13] Sir H. Lamb. Hydrodynamics. University Press, Cambridge, 4th edition, page 450, 1916.

[14] P. G. de Gennes, F. Brochard-Wyart, D. Quéré, and B. Widom. Capillarity and Wetting Phenomena: Drops, Bubbles, Pearls, Waves. Springer-Verlag, 2004. ISBN 9780387005928.

[15] S. F. Jones, G. M. Evans, and K. P. Galvin. Bubble nucleation from gas cavities - a review. J. Coll. Interf. Sci., 80(1):27 - 50, 1999.

[16] G. Liger-Belair, Mi. Vignes-Adler, C. Voisin, B. Robillard, and P. Jeandet. Kinetics of gas discharging in a glass of champagne: The role of nucleation sites. Langmuir, 18(4):1294-1301, 2002.

[17] P. M. Morse and H. Feshbach. Methods of Theoretical Physics. McGrawHill Book Company Inc., 1953.

[18] B. Cuenot, J. Magnaudet, and B. Spennato. The effects of slightly soluble surfactants on the flow around a spherical bubble. J. Fluid Mech., 339: 25-53, 1997.

[19] G. B. Arfken, H. J. Weber, and L. Ruby. Mathematical methods for physicists, 4th ed. American Journal of Physics, 64(7):959, 1996.

[20] L.F. Shampine. Vectorized adaptive quadrature in matlab. Journal of Computational and Applied Mathematics, 211(2):131 - 140, 2008.

[21] L .E. Kinsler, A. R. Frey, A. B. Coppens, and J. .V. Sanders. Fundamentals of acoustics. John Wiley \& Sons. Inc, 2000. ISBN 0-471-84789-5.

[22] M. Versluis, D. E. Goertz, P. Palanchon, I. L. Heitman, S. M. van der Meer, B. Dollet, N. de Jong, and D. Lohse. Microbubble shape oscillations excited through ultrasonic parametric driving. Phys. Rev. E, 82(2):026321, 2010. 


\section{3}

\section{Illumination by Laser-Induced Fluorescence for single flash imaging on a nanoseconds timescale ${ }^{1}$}

The challenge in visualizing fast microscale fluid motion phenomena is to record high quality images free of motion blur. Here we present an illumination technique based on laser-induced fluorescence which delivers high intensity light pulses of $7 \mathrm{~ns}$. The light source consists of a Q-switched Nd:YAG laser and a laser-dye solution incorporated into a total internal reflection lens, resulting in a uni-directional light beam with a millimeter-sized circular aperture and $3^{\circ}$ divergence. The laser coherence, considered undesirable for imaging purposes, is reduced whilst maintaining a nanoseconds pulse duration. The properties of the illumination by laser-induced fluorescence (iLIF) are quantified and a comparison is made with other high-intensity pulsed and continuous light sources.

\subsection{Introduction}

It has been a challenge throughout the last century to freeze fast fluid motion phenomena onto a single image and, in such a way, distinguish the finest details at the smallest length scales. One of the first to address this challenge was A.M. Worthington [1] in 1895 when he used a spark to visualize a splash of milk. Worthington already predicted that flash recording would become of great importance in scientific research, but it wasn't until the work of Edgerton [2] that the scientific utilization of flash photography became widely accepted. In the last decade digital camera technology has evolved rapidly, and high-speed visualization has become a valuable experimental method, see e.g. Thoroddsen

\footnotetext{
${ }^{1}$ Published as: Arjan van der Bos, Aaldert G. Zijlstra, Erik Gelderblom, Michel Versluis iLIF: illumination by Laser-Induced Fluorescence for single flash imaging on a nanoseconds timescale, Experiments in Fluids, 51 (1), 1-7, 2011
} 
et al. 3] At the same time, illumination techniques continued to develop, from high-intensity Xenon light sources to low cost high intensity LEDs, both of which can be used for pulsed as well as continuous illumination.

In microfluidics a wide range of phenomena are studied, including the dynamics of droplets, bubbles and particles. Despite the small sizes of these objects the associated velocities are usually in the order of meters per second. Hence, to achieve accurate visualization not only a high spatial resolution but also a high temporal resolution is required. For this purpose one can either employ a camera with a short exposure time, or a light source capable of emitting flashes of short duration. The first option generally requires a fast (and expensive) high-speed camera, which is often limited in its pixel resolution [3]. Therefore flash photography is the preferred method for experiments where high-resolution single images are required, since the method does not require a fast shutter and can in principle be performed with any type of camera. Furthermore the pulse duration of flash illumination sources can easily be shorter than the shortest exposure time of a high-speed camera. On the other hand, the repetition rate of high-intensity flash light sources is often limited to several kilohertz, making these sources unsuitable for recording multiple consecutive frames at microseconds or shorter timescales.

Nowadays, several types of nanosecond light sources are available that can deliver a considerable amount of light within a few nanoseconds. There are, for example, affordable solutions with high intensity LEDs [4-6], which can deliver up to $10^{12}$ photons per flash of $2 \mathrm{~ns}$, thus close to $1 \mu \mathrm{J}$ per flash. A comparable, but more expensive solution is using high-intensity spark plugs [7, 8]. However, when it comes to emitting ultrashort high-intensity light flashes, a pulsed laser is clearly the most effective. The duration of laser pulses are typically in the order of nanoseconds, but can be as short as attoseconds [9]. Lasers can be used directly for flash illumination, as was done for example by Dong et al. [10] and Juhasz et al. [11], but there are some major drawbacks associated with a fully coherent light source. The light emitted by a laser has a very narrow bandwidth and has a long coherence length, resulting in diffraction fringes and laser speckle patterns in the recorded images [11]. Additionally, spurious reflection of the laser beam has the potential risk of causing permanent damage to the camera sensor. Methods to suppress these effects are predominantly based on reducing the spatial coherence the light, for example by applying rotating or vibrating optical elements like fibers, wobblers and diffusers [12]. A good example to obtain this was proposed by Dingel and Kawata [13], where the laser light is coupled into multiple fibers with different lengths, after which the light from the separate fibers is recombined and passes through a spatial diffuser (brand: Nanophoton). For a laser pulse however, a more effective method 


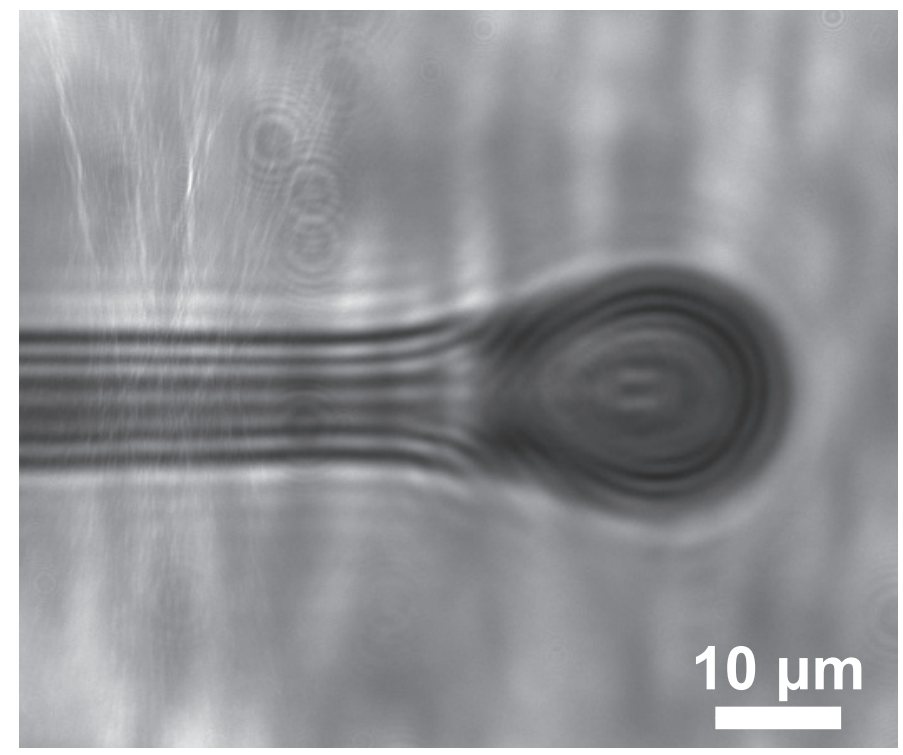

Figure 3.1: The head of an inkjet droplet moving from left to right with a velocity close to $6 \mathrm{~m} / \mathrm{s}$. This image was made using the illumination where the laser dye is contained in a cuvette as described in chapters 4 and 5 of this thesis. The image shows a speckle pattern in the background and interference patterns of the light passing through and around the droplet.

is to decrease its temporal coherence. One interesting approach to achieve this is through excitation of a fluorescent laser dye. Here the pulse duration is practically unaltered, but the energy of the pulse is redistributed into a broader optical bandwidth at higher wavelengths. This technique has proven to be very effective and was already applied in shadowgraph experiments (see next chapter of this thesis), and is closely related to fluorescent flow visualization described in [14, 15]. A disadvantage is that the cuvette containing the dye can act as a secondary laser cavity [16], thereby partially restoring the coherent nature of the pulse (see Fig. 3.1). Additionally, the intensity profile of the emitted light is asymmetric which complicates obtaining homogeneous illumination.

Here we address these issues using a modified single flash illumination technique based on laser-induced fluorescence. The obtained illumination is ideally suited for flash-photography in experiments involving timescales of order microseconds in conjunction with length scales of order micrometer. To validate the technique its performance is compared in a standard imaging setup with a range of high-end commercially available light-sources, both pulsed and continuous. 


\subsection{Criteria for high-speed flash-photography}

The application of flash-photography in experiments is primarily aimed at obtaining precise information about the position and dimensions of the studied object at a certain instant in time. It is therefore of prime importance to capture the smallest details in both temporal- and spatial resolution at high contrast. To define this more quantitatively three criteria are stated. These are the spatial Nyquist criterion, maximizing the signal-to-noise ratio of the CCD and minimizing motion blur.

First, assuming that a microscope objective with the highest possible numerical aperture is chosen for the experiment, the effective optical magnification $M_{\text {eff }}$ must be sufficient to avoid undersampling of the image with respect to the spatial resolution $r$ as defined by the Rayleigh-criterion [17]. The spatial Nyquist criterion requires that at least 2 pixels lay within $r M_{\text {eff }}$ hence it follows that the magnification must be such that

$$
M_{\text {eff }}>\frac{2 d}{r}
$$

where $d$ is the pixel size. Larger magnifications allow denser spatial sampling (known as oversampling) but also result in a smaller field-of-view and lower image brightness. The magnification is therefore limited by the optimum size of the object-image and the available illumination intensity.

Secondly, to ensure maximum image contrast the intensity of the illumination should be adjusted to cover the full dynamic range of the camera sensor. The extent to which the intensity can be varied is, however, limited due to the reciprocal relationship between illumination intensity and exposure time. To clarify, an image with a certain fixed brightness can be obtained with, either low light conditions and longer exposure times, or vice versa. In the situation where an object is motionless both settings result in identical images. However if the object of interest is moving, the image becomes susceptible to motionblur. This undesired effect causes a smeared appearance of the image of the object due to its displacement during the time the image is recorded.

Minimizing motion-blur constitutes the third criterion which is required to accurately capture a single high-resolution image of a moving object and this can be achieved by adjusting the temporal resolution of the imaging system. As discussed, this temporal resolution is determined either by the duration of the illumination pulse (indicated by $\tau_{p}$ ) or by the camera exposure time $\tau_{c}$, which is the time duration during which the mechanical- or electronic shutter of the camera is opened to expose the sensor to an illuminated object. Evidently, the actual temporal resolution $\tau$ will be the shortest of both durations, hence

$$
\tau=\min \left(\tau_{p}, \tau_{c}\right) .
$$




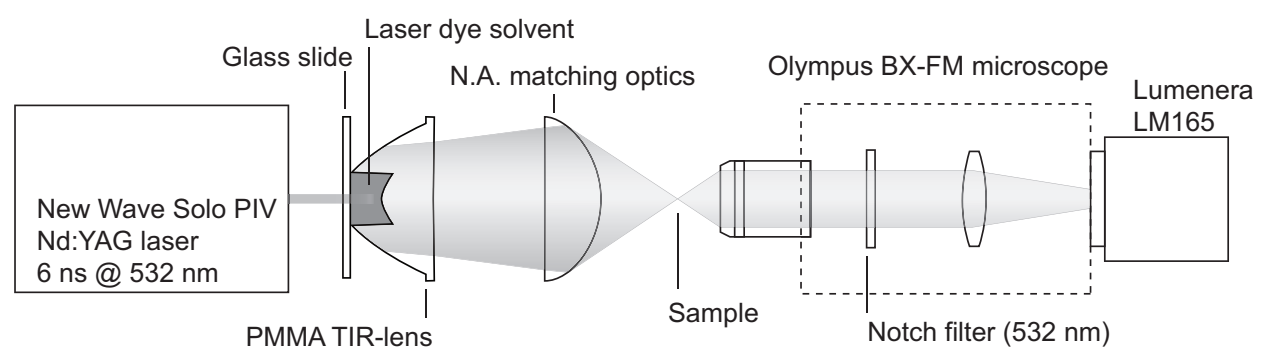

Figure 3.2: Typical application of illumination with laser dye fluorescence. In the illumination setup a laser excites the dye after which the fluorescent light from the cavity is collected and focused through a collimation lens onto a microscope. To filter out any residual laser light a notch filter (Semrock NF01-532U-25) is used.

If we now define $\varepsilon$ as being the displacement in pixels of the object moving with velocity $U$ during time $\tau$ :

$$
\varepsilon=\tau \frac{M_{\mathrm{eff}} U}{d}
$$

motion-blur will be minimal if $\varepsilon \leq 1$. In most experiments the object velocity, magnification and pixel-size are pre-determined and motion-blur is minimized by choosing an illumination or exposure time such that

$$
\tau \leq \frac{d}{M_{\mathrm{eff}} U}
$$

\subsection{Illumination by laser induced fluorescence}

To obtain maximum fluorescence efficiency while minimizing coherence we constructed a laser-dye lens. The core of a total internal reflection lens (TIR-lens) where normally a high-power LED can be placed was filled with a laser-dye (Fig. 3.2). This dye consisted of a fully saturated solution of LDS 698 (Exciton Inc., Dayton, U.S.) in ethanol. The TIR-lens (L2-Optics) is an acrylic collimator with a total beam divergence of $3^{\circ}$. The dye was excited by a Nd:YAG laser (Solo PIV, New Wave) emitting a 6.5 ns pulse (full width at half maximum, see Figure 3.3) with a maximum energy of $100 \mathrm{~mJ}$ at $532 \mathrm{~nm}$. This configuration allows the greater part of the laser pulse to be absorbed, re-emitted and subsequently reflected in the forward direction thus promoting the reduction of the temporal coherence of the pulse.

To confirm the applicability of this setup for illumination by laser induced fluorescence (iLIF), the pulse length and spectrum were measured. To measure the pulse length a photodiode (Thorlabs-DET 210) was mounted directly 


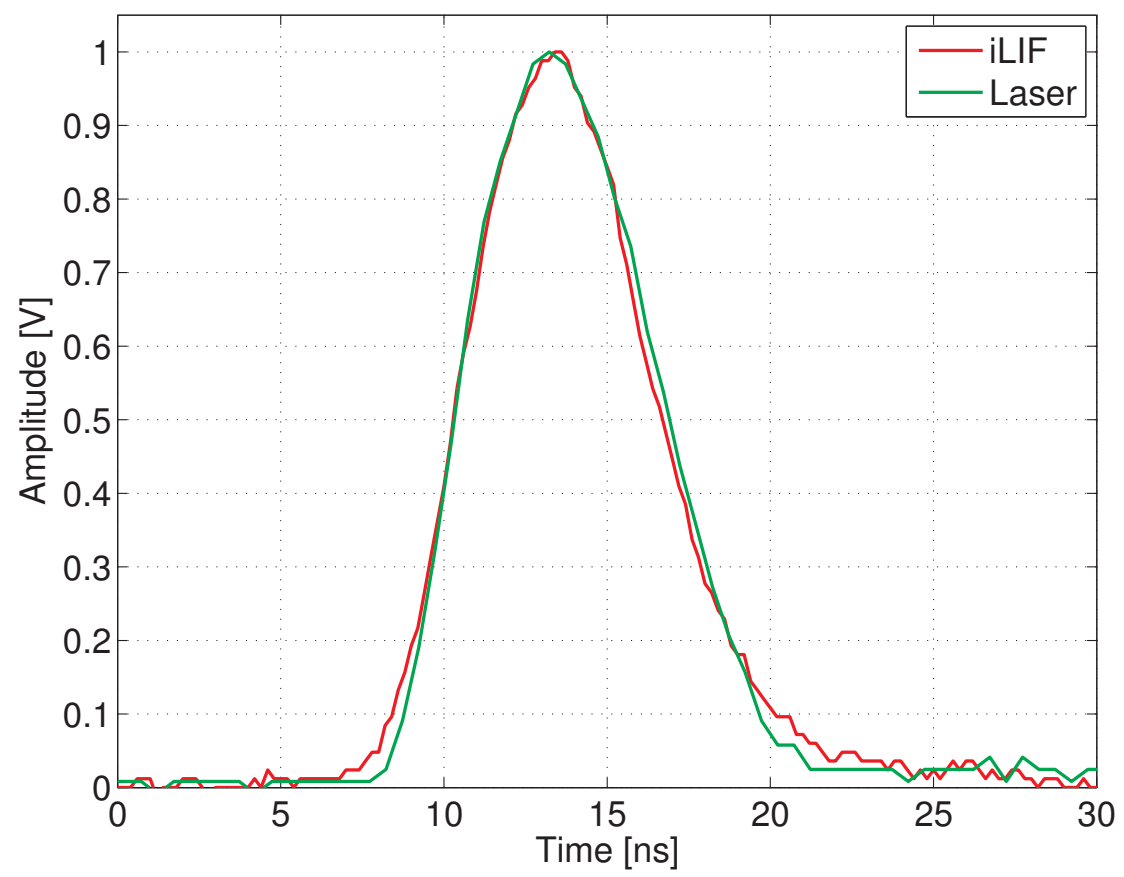

Figure 3.3: The normalized average of 16 measurements of the pulse shape of both the laser- and the iLIF pulse.

after the TIR-lens and connected to an oscilloscope (Tektronix TDS5034B), resulting in a typical rise time of the measuring system of $1.2 \mathrm{~ns}$. The laser and oscilloscope were triggered with a pulse/delay generator (Berkely Nucleonics model 565) with an accuracy of 250 ps. The measurement was carried out with- and without a notch filter in between the lens and photodiode. As shown in Figure 3.3 the iLIF does not result in a noticeable difference in pulse length with respect to the laser. The influence of the notch filter on the iLIF-pulse shape was also found to be negligible.

The spectral characteristics of the iLIF pulse were determined using a highresolution fiber optic spectrometer (AVASPEC 3648, FWHM resolution: 0.32 $\mathrm{nm}$ ). Figure 3.4 shows the broad spectrum of the fluorescence light. The spectrum was recorded with the notch filter in front of the spectrometer. However, measurements without the notch filter also did not show a peak at $532 \mathrm{~nm}$ indicating that most of the laser energy is absorbed by the laser dye. 


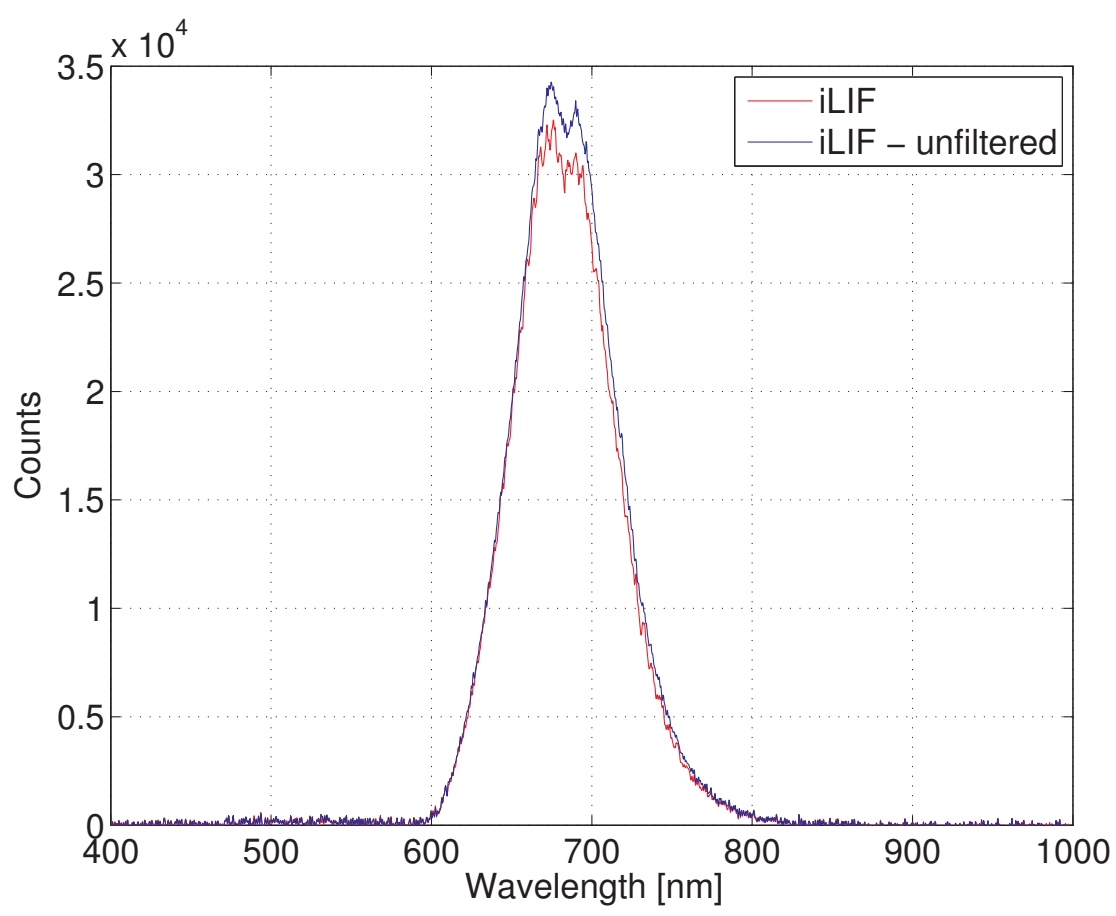

Figure 3.4: Spectra of the iLIF pulse with- and without the notch filter.

\subsection{Comparison with other light sources}

To quantify the performance of the laser-induced fluorescence illumination a comparison was made with several high-intensity light sources, typically used for high-speed imaging of microscopic events. An overview of the light sources, both pulsed and continuous, is given in Table 3.1. All continuous light sources have an optical fiber, glass or liquid core with a diameter of 4 to $8 \mathrm{~mm}$, to deliver the light to the sample, except for the LED's and the continuous xenon light source. The LED's are mounted in a TIR-lens with a divergence of $3^{\circ}$, comparable to the TIR-lens used for iLIF. The continuous xenon light source (Hella B.V.) has an aperture with a radius of approximately $10 \mathrm{~cm}$.

The test setup is schematically depicted in Figure 3.5 and was designed to measure the relative intensity of all the light sources. Since the light source properties such as the beam profile, beam divergence and spectral intensity profile vary from one light source to the other, the setup was chosen to resemble a typical but simplified experimental setup for high-speed flow visualization. An aspheric lens pair collimated the light from the source onto a USAF 1951 


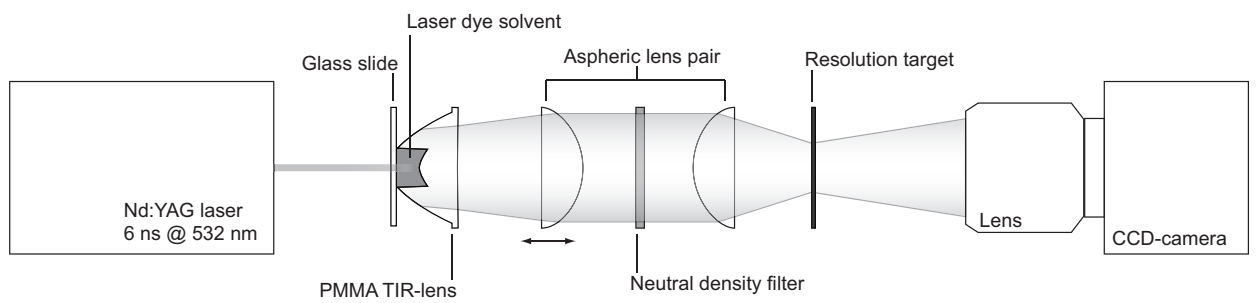

Figure 3.5: Setup used for the comparison of the various light sources. The location of the first aspheric lens is adjusted to collect and collimate the light from the lightsource.

resolution test chart (Edmund Optics). A telecentric lens (Jenoptik) was used to form a 1:1 image of a $500 \times 500 \mu \mathrm{m}^{2}$ square from the target onto a CCDcamera (Lumenera, LM165M, 8 bit monochrome). Neutral density filters with optical densities ranging from 2.5 to 5.0 were used to avoid overexposure of the CCD. Except for the first aspheric lens, all elements of the setup and the position of the light source were fixed in place. To optimize the light intensity and to ensure even illumination of the target, the position of the movable lens was adjusted for each light source.

For the continuous light sources the exposure time of the camera was adjusted to get an average pixel value of the image of the square of approximately 200

Table 3.1: Characteristics of the light sources

\begin{tabular}{llll} 
Light source & Type & $\begin{array}{l}\text { Pulse } \\
\text { length } \\
\text { (fwhm) }\end{array}$ & $\begin{array}{l}\text { Pulse } \\
\text { rate } \\
\text { (max) }\end{array}$ \\
\hline \hline Laser Dye TIR lens & pulsed & $7 \mathrm{~ns}$ & $20 \mathrm{~Hz}$ \\
Laser Dye cuvette & pulsed & $7 \mathrm{~ns}$ & $20 \mathrm{~Hz}$ \\
HSPS Nanolite KL-L & pulsed & $16 \mathrm{~ns}$ & $20 \mathrm{kHz}$ \\
Seoul Semicon P7 & pulsed/CW $1 \mu \mathrm{s}$ & $1 \mathrm{MHz}$ \\
Lumiled Luxeon Star & pulsed/CW $400 \mathrm{~ns}$ & $1 \mathrm{MHz}$ \\
Perkin Elmer MVS-7010 & pulsed/CW & $200 \mu \mathrm{s}$ & $10 \mathrm{~Hz}$ \\
Olympus ILP-1 & $\mathrm{CW}$ & - & - \\
Sumita LS-M352 & $\mathrm{CW}$ & - & - \\
Schott KL1500LCD & $\mathrm{CW}$ & - & - \\
Schott Ace & $\mathrm{CW}$ & - & - \\
Hella Mega Beam Xenon & $\mathrm{CW}$ & - & - \\
\hline
\end{tabular}




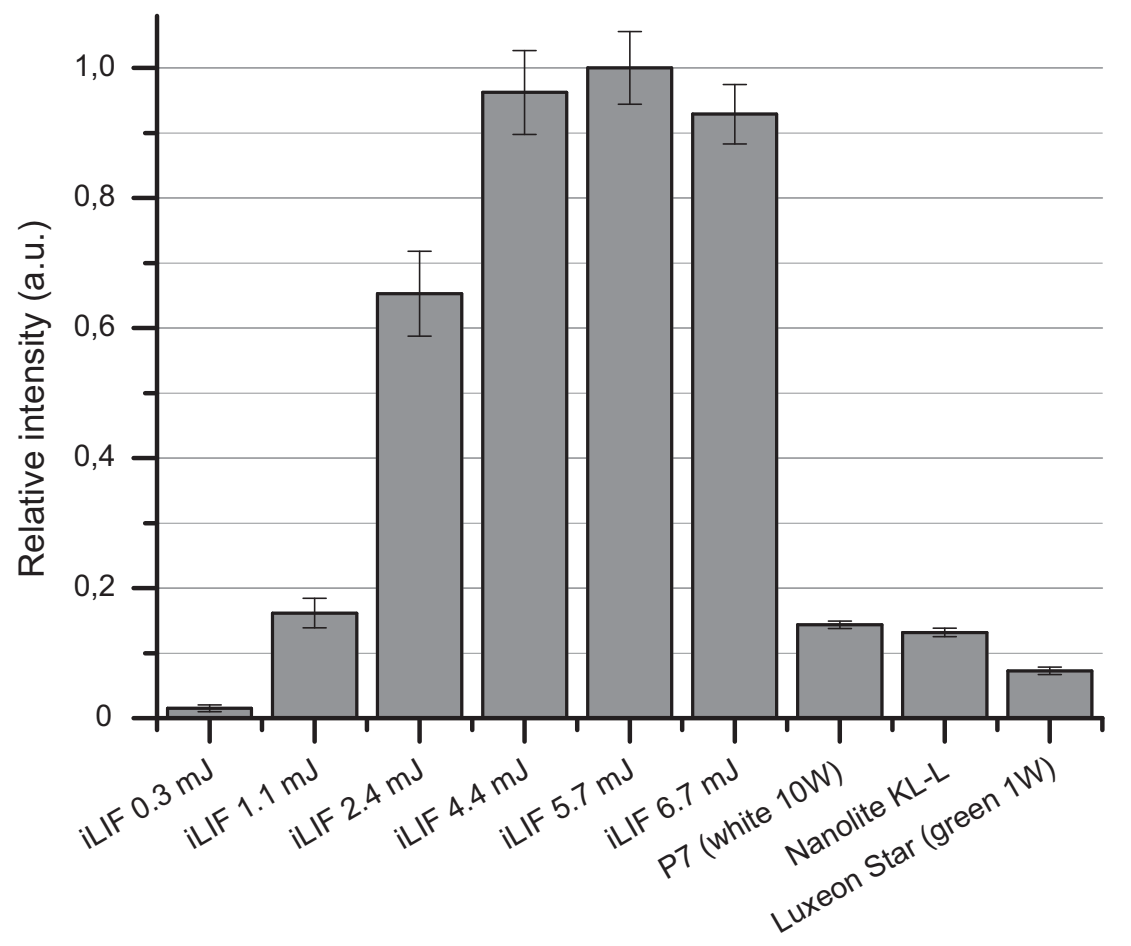

Figure 3.6: Relative intensity of the pulsed lightsources. The energy values indicate the laser pulse energy used to excite the laser-dye.

out of 255 counts. This procedure was repeated for several ND-filters per light source. The recorded images were analyzed and the results were scaled with the exposure times, the number of pulses and the optical density of the ND-filters used.

Figure 3.6 shows the comparison of the single pulse intensities of the pulsed light sources. Saturation behavior of the fluorescent laser-dye is visible in the graph as the maximum light intensity peaks at a laser energy of $5.7 \mathrm{~mJ}$. Compared to the LEDs the iLIF shows a sixfold higher light intensity at a pulse length which is two orders of magnitude shorter. The Nanolite has a light intensity comparable to the LEDs but with a pulse length similar to the iLIF. However, the location of the spark from the Nanolite varies per shot, causing large variations in illumination intensity and inhomogeneities between images.

The results of the continuous light sources were scaled to an illumination time of 7 ns to match the pulse length of the iLIF and compared in Figure 3.7. From the graph the potential of the iLIF becomes clear; the semi-continuous 


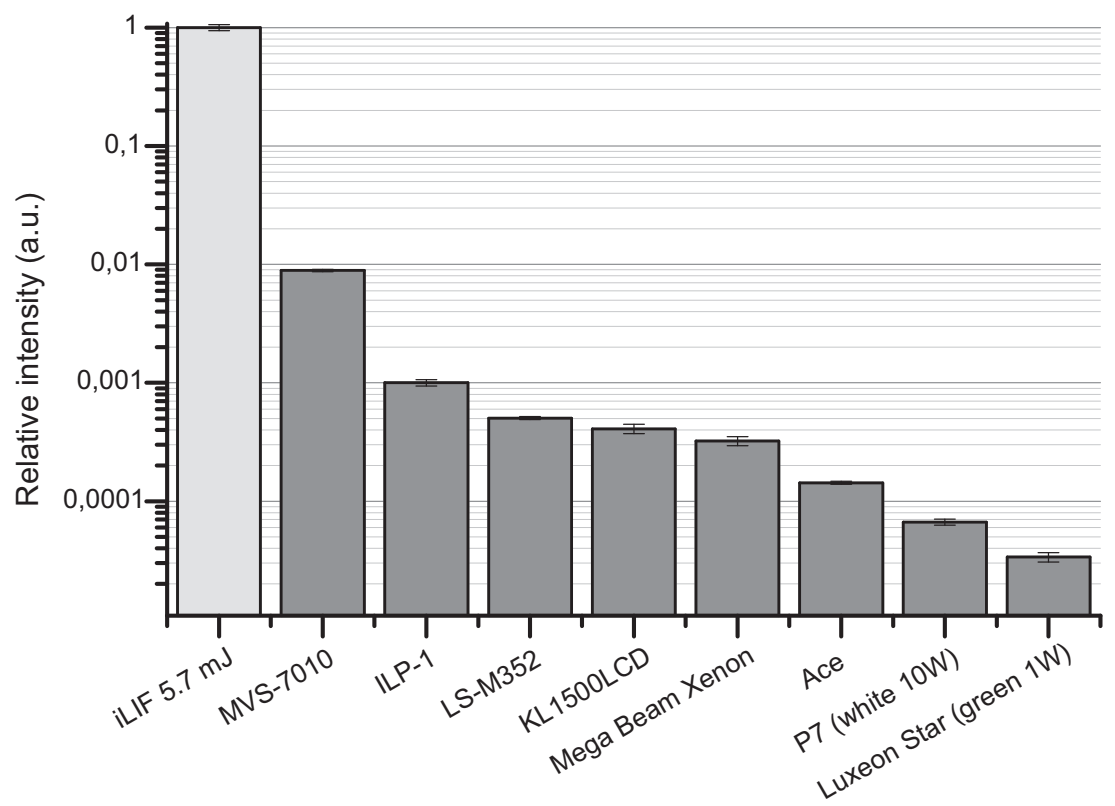

Figure 3.7: Relative illumination intensities for the continuous lightsources normalized to the maximum obtainable intensity of the iLIF and scaled to 7 ns illumination time. Note the logarithmic scale.

xenon flash is outperformed by two orders of magnitude and the continuous light sources are even a factor of one thousand less intense than the iLIF. In future research it would be interesting to compare the iLIF technique with illumination by a copper vapor laser (Oxford Lasers). Such lasers generate pulses with a relative low coherence at a very high pulse repetition frequency. However, drawbacks of these lasers are increased pulse length, lower pulse energy, and higher costs.

\subsection{Examples}

As drop formation in inkjet printing is an almost perfectly reproducible process, single flash photography is frequently applied for its visualization [10, 18]. Here we use this reproducible quality to demonstrate the effectiveness of the iLIF compared to two other flash illumination sources. These are the highintensity Luxeon $L E D$ and the HSPS Nanolite $K L-L$ with pulse durations of $\tau_{p}=400 \mathrm{~ns}$ and $\tau_{p}=16 \mathrm{~ns}$ respectively. The experimental setup used was 
equivalent to the setup shown in Figure 3.2, where the inkjet print head is placed at the sample location. The incident light is focused through a collimation lens onto the region of interest. The collimation lens was chosen such that the numerical aperture (NA) matches with that of the microscope objective ensuring maximal optical resolution $(r \approx 2 \mu \mathrm{m})$ and intensity [17]. The microscope (Olympus BX-30MF) is equipped with three long working distance (WD) objectives, the LMPLFLN 10×, (Olympus, WD $21 \mathrm{~mm} / \mathrm{NA}=0.25)$, the SMPLFLN 20× (Olympus, WD $25 \mathrm{~mm} / \mathrm{NA}=0.25$ ), and the SMPLFLN 50×, (Olympus, WD $18 \mathrm{~mm} / \mathrm{NA}=0.35$ ). A notch filter (Semrock, NF01-532U-25) is placed before the camera to filter out any residual laser light. The camera is a Lumenera LM165 with a sensitive Sony EXview HAD CCD sensor with pixel size of $6.45 \times 6.45 \mu \mathrm{m}^{2}$, and a resolution of $1392 \times 1040$. The scale factors for the camera and the $10 \times, 20 \times$ or the $50 \times$ microscope objective combination are $640 \mathrm{~nm} /$ pixel, $320 \mathrm{~nm} /$ pixel, or $129 \mathrm{~nm} /$ pixel respectively.

The printhead is an experimental prototype developed by Océ Technologies B.V., similar to the printheads used in references [19, 20]. The printhead ejects droplets with a diameter of $30 \mu \mathrm{m}$ at a typical velocity of $20 \mathrm{~m} / \mathrm{s}$ during drop formation. Some $35 \mu$ s later the tip of the droplet reaches a terminal velocity close to $6 \mathrm{~m} / \mathrm{s}$. Both stages were imaged with each of the three illumination sources (see Fig. 3.8). The left column shows the first stage of drop formation imaged with the $10 \times$ objective. The second stage where the droplet has just exited the nozzle and has slowed down to its terminal velocity is shown in the middle column, imaged with the $20 \times$ objective. The column on the right shows an enlarged view of the region where the droplet pinches off from the meniscus, imaged with the $50 \times$ objective.

The practical advantages of the combination of high-intensity and short pulses of light for imaging of fast phenomena become apparent from a detailed comparison of the images in Fig. 3.8. First, unlike the images taken with the iLIF pulse, the images in Fig. $3.8 \mathrm{~b}$ and Fig. 3.8k required a maximal CCD gain factor of 24 for the $50 \times$ objective. As a consequence these images have much lower contrast and a grainy appearance due to the higher noise level. This is further illustrated in the graphs of Fig. 3.9. Even though the images of the droplet made with the Nanolite and the iLIF appear of similar quality, Fig. 3.9 clearly shows that the signal-to-noise level is higher for the iLIF method.

Secondly, using equation (3.4) for the indicated magnifications we find that the droplet can only be imaged without motion-blur if the illumination times are smaller than $\tau_{10}=32 \mathrm{~ns}, \tau_{20}=16 \mathrm{~ns}$ and $\tau_{50}=6 \mathrm{~ns}$. The droplet displacement during exposure $\varepsilon$ is acceptable for both the iLIF and the Nanolite (see table 3.2), however the pulse length of the LED is too long, resulting in motion-blur as can readily be seen in Fig. 3.8. 


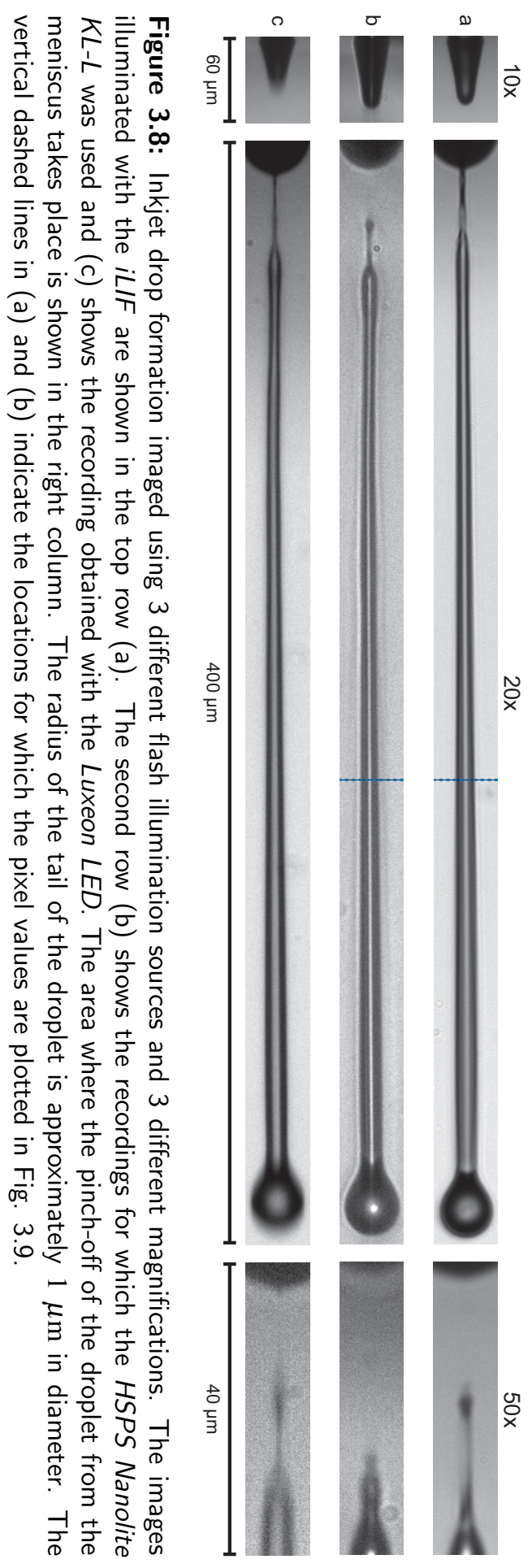


(a) iLIF

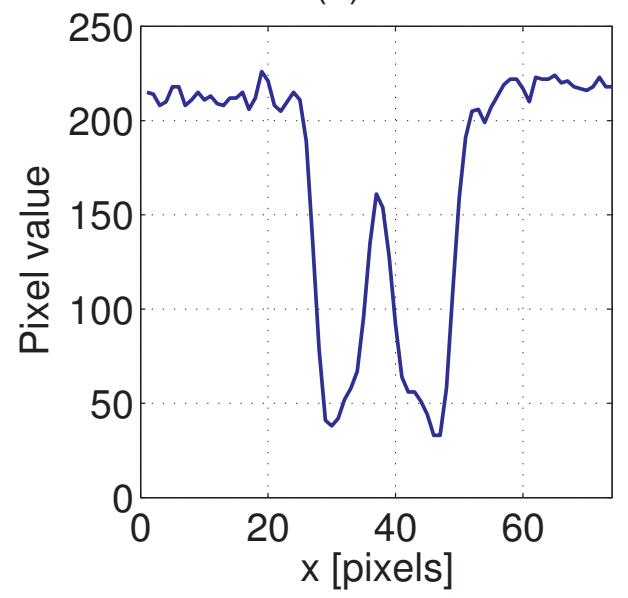

(b) Nanolite

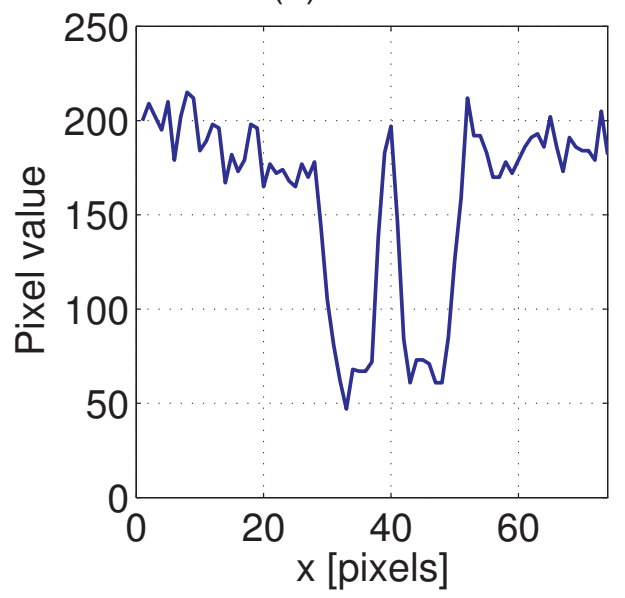

Figure 3.9: Pixel values along the dashed lines in the images in Fig. 3.8 and 3.8p. The higher signal-to-noise ratio in the image made with iLIF compared to that of the HSPS Nanolite $K L-L$ enables a more precise determination of the droplet dimensions.

Table 3.2: Comparison of the amount of motion-blur indicated by $\varepsilon$ and the relative light intensity for the imaging of a $20 \mathrm{~m} / \mathrm{s}$ inkjet droplet using three pulsed light sources.

\begin{tabular}{l|l|l|l} 
Light source & $M_{\text {eff }}$ & $\varepsilon$ & $\begin{array}{l}\text { Rel. } \\
\text { Inten- } \\
\text { sity }\end{array}$ \\
\hline \hline Laser Dye TIR lens $\left(\tau_{p}=7 \mathrm{~ns}\right)$ & 10 & 0.22 & 1.0 \\
& 20 & 0.43 & 1.0 \\
HSPS Nanolite KL-L $\left(\tau_{p}=16 \mathrm{~ns}\right)$ & 50 & 1.1 & 1.0 \\
\hline \multirow{2}{*}{ Lumiled Luxeon Star $\left(\tau_{p}=400 \mathrm{~ns}\right)$} & 10 & 0.47 & 0.16 \\
& 50 & 0.93 & 0.03 \\
& 10 & 12.3 & 0.02 \\
\hline
\end{tabular}




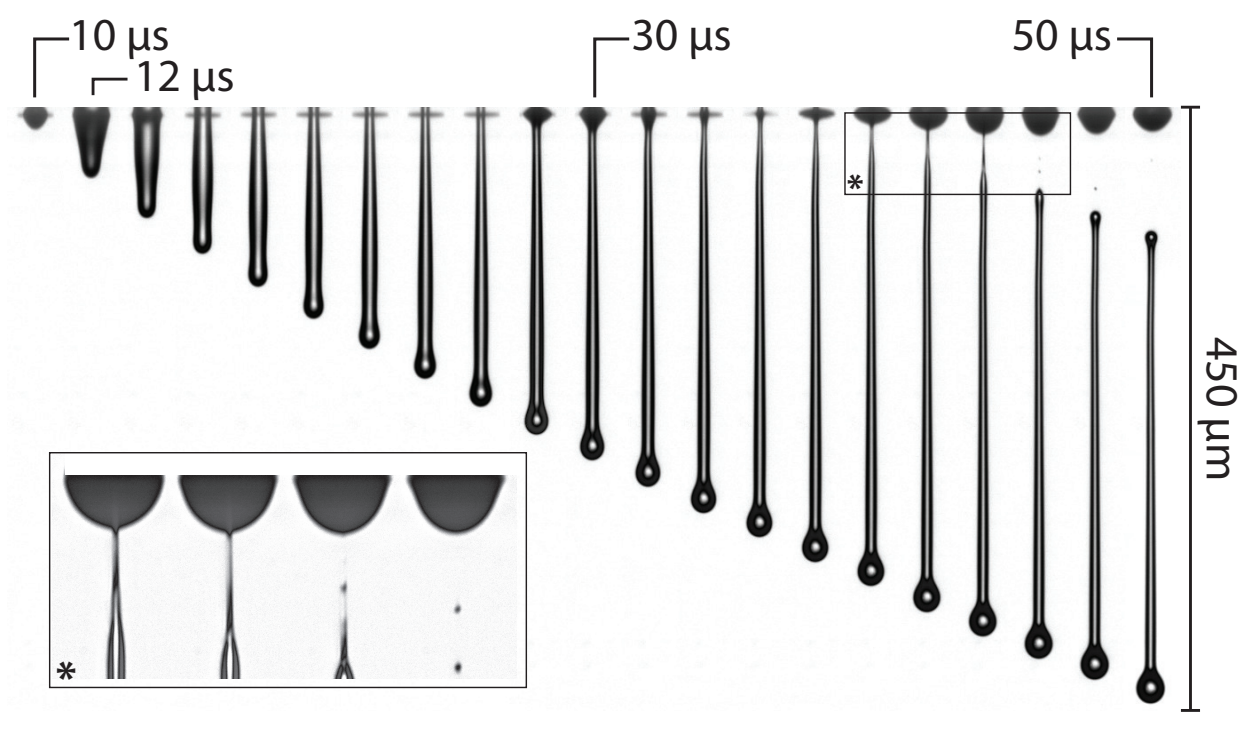

Figure 3.10: Inkjet drop formation as a function of time. Here 21 individual droplets are shown which illustrate the reproducibility of the experiment and the quality of the iLIF light source. The inset shows an enlarged view of the area where the droplet pinches off from the meniscus. The background has been subtracted to create a higher contrast.

Evidently, the characteristics of the iLIF allow both high contrast and small time resolution imaging. This is also demonstrated in Figure 3.10 showing the evolution of the inkjet droplet recorded with the iLIF. The images reveal the size of a thin secondary tail between the drop and meniscus (also seen in Figure 3.8F). The improved quality of the recordings, both in spatial and temporal resolution, give way to novel analysis of the drop formation, as is elaborately discussed in [21].

\subsection{Discussion and conclusion}

The iLIF fluorescence illumination method presented here proves to be a very promising technique for flash photography of fast and small scale phenomena. The illumination technique was evolved from an earlier applied technique were a fluorescent dye in a cuvette was used to reduce the coherence of the laser illumination. Unlike the cuvette, the geometry of the TIR-lens avoids secondary laser emission and it directs the light uniformly in the forward direction. In this way the iLIF system emits 7 ns light pulses with low temporal coherence, 
thereby avoiding unfavorable effects such as speckle and diffraction. Compared to other high-intensity pulsed and continuous light sources it shows significantly higher intensity levels with a short pulse duration, uniform illumination and low intensity jitter.

The characteristics of this method of illumination are determined by the properties of the laser and dye combination and the TIR-lens, which were not optimized until now. Hence it is clear that there is plenty of space for improvements or adjustments to obtain shorter pulse lengths, higher efficiency, different wavelengths or larger apertures. The latter could be very useful for the visualization of macro scale experiments like impact phenomena and shock waves in fluids. 


\section{Bibliography}

[1] A. M. Worthington. The splash of a drop. New York: E. \& J. B. Young \& CO., 1895.

[2] H. E. Edgerton and J. R. Killian. Moments of vision : the stroboscopic revolution in photography. MIT Press, Cambridge, Mass., 1979.

[3] S. T. Thoroddsen, T. G. Etoh, and K. Takehara. High-Speed Imaging of Drops and Bubbles. Ann. Rev. Fluid Mech., 40(1):257-285, 2008.

[4] R. V. Vasiliev, B. K. Lubsandorzhiev, and P. G. Pokhil. A nanosecond light source for scintillation-and Cerenkov-detector calibration. Instrum. Exp. Tech. (USSR), 43(4):570-572, 2000.

[5] C. N. Scholfield and M. Murdock. Pulse-modulated light source for psychometric and vision experiments. J. Neurosci. Methods, 19(3):203-207, 1987.

[6] B.K. Lubsandorzhiev, R.V. Poleshuk, B.A.M. Shaibonov, and Y.E. Vyatchin. LED based powerful nanosecond light sources for calibration systems of deep underwater neutrino telescopes. Nucl. Instrum. Methods. Phys. Res. A, 602(1):220-223, 2009.

[7] S. Miyashiro and H. Grnig. Low jitter reliable nanosecond spark source for optical short-duration measurements. Exp. Fluids, 3:71-75, 1985.

[8] High Speed Photo Systeme - Nanolite. Von-Linne-Str. 12, D-22880 Wedel, Germany. http://www.hsps.com/

[9] E. J. Takahashi, P. Lan, O. D. Mücke, N. Yasuo, and M. Katsumi. Infrared Two-Color Multicycle Laser Field Synthesis for Generating an Intense Attosecond Pulse. Phys. Rev. Lett., 104(23):233901, 2010.

[10] H. Dong, W. W. Carr, and J. F. Morris. Visualization of drop-on-demand inkjet: Drop formation and deposition. Rev. Sci. Instr., 77(8):085101, 2006.

[11] T. Juhasz, G. A. Kastis, C. Suárez, Z. Bor, and Walter E. Bron. Timeresolved observations of shock waves and cavitation bubbles generated by femtosecond laser pulses in corneal tissue and water. Lasers Surg. Med., 19(1):23-31, 1996.

[12] T. Iwai and T. Asakura. Speckle reduction in coherent information processing. Proc. IEEE, 84(5):765-781, 1996. 
[13] B. Dingel and S. Kawata. Speckle-free image in a laser-diode microscope by using the optical feedback effect. Opt. Lett., 18(7):549-551, 1993.

[14] K. R. Rau, P. A. Quinto-Su, A. N. Hellman, and V. Venugopalan. Pulsed Laser Microbeam-Induced Cell Lysis: Time-Resolved Imaging and Analysis of Hydrodynamic Effects. Biophys. J., 91(1):317 - 329, 2006.

[15] J. W. Shan, D. B. Lang, and P. E. Dimotakis. Scalar concentration measurements in liquid-phase flows with pulsed lasers. Exp. Fluids, 36:268-273, 2004.

[16] F. P. Schäfer, W. Schmidt, and J. Volze. Organic dye solution laser. Applied Physics Letters, 9(8):306-309, 1966.

[17] M. W. Davidson and M. Abramowitz. Optical microscopy. Encyclopedia of Imaging Science and Technology, 2:1106-1141, 2002.

[18] I. M. Hutchings, G. D. Martin, and S. D. Hoath. High speed imaging and analysis of jet and drop formation. J. Imaging Sci. Technology, 51(5):438, 2007.

[19] J. de Jong, G. de Bruin, H. Reinten, M. van den Berg, H. Wijshoff, M. Versluis, and D. Lohse. Air entrapment in piezo-driven inkjet printheads. J. Acoust. Soc. Am., 120(3):1257, 2006.

[20] H. Wijshoff. The dynamics of the piezo inkjet printhead operation. Phys. Rep., 491(4-5):77-177, 2010.

[21] J. A. van der Bos. Air entrapment and drop formation in piezo inkjet printing. PhD thesis, University of Twente, The Netherlands, 2011. 


\section{4}

\section{Fiber optic probe hydrophone measurements in a cavitating liquid ${ }^{1}$}

The measurement of high-pressure signals is often hampered by cavitation activity. The usage of a fiber optic probe hydrophone is becoming the gold standard to measure in this harsh environment. Yet, when measuring in a cavitating liquid large variations in the signal amplitude are found, in particular when the pressure signal recovers back to positive values. Here, we photograph with ultrashort flash photography the wave propagation and bubble dynamics and reveal the importance of secondary shock waves emitted from collapsing cavitation bubbles. Interestingly, just adding a small amount of acidic acid reduces the cavitation activity to large extend. With this treatment we find an altered primary pressure profile which does not force the cavitation bubbles close to fiber tip into collapse. Thereby, the shot-to-shot variations are greatly reduced.

\subsection{Introduction}

Accurately registering the wave forms in medical applications such as in shock wave lithotripsy (SWL) [1, 2], shock wave therapy (SWT) [3], histotripsy [4, or high intensity focused ultrasound (HIFU) [5] is of prime importance for quality assurance of therapeutical devices. Pressure measurements of high-pressure finite amplitude and shock waves are not only demanding because of the high frequencies involved. The recording of negative pressures is also challenging because the sensor might have to withstand cavitation [6]. In general cavitation can occur when the pressure drops below the vapor pressure while nuclei [7] are present which explode into vaporous cavities. When the pressure recovers

\footnotetext{
${ }^{1}$ Published as: Aaldert G. Zijlstra \& Claus-Dieter Ohl, On fiber optic probe hydrophone measurements in a cavitating liquid, Journal of the Acoustical Society of America 123, pp. 29-32 (2008).
} 
again the cavities implode thereby focusing "destructive" energy from the liquid onto very small scales. When the sensor is too close to the violent collapse of a cavity it can easily be damaged. Yet, even if the sensor withstands the cavity collapse, measurements of the pressure are often hampered because of the need to distinguish whether the sensor is entrained within a cavity or accurately registering the liquid pressure.

A device which operates in this demanding environment is the fiber optic probe hydrophone developed by Staudenrauss and Eisenmenger (1992) [8]. In this device laser light is coupled into a glass fiber. At the fiber tip the light is reflected; the intensity of the reflected light is a function of the jump in the index of refraction from glass to water. The index of refraction is related through the well known Gladstone-Dale relationship to the pressure. Thus, the pressure can be determined from the intensity reflected back into the fiber [9] and registered with a sensitive photodetector. This type of hydrophone is mentioned in the IEC guidelines for quality assurance of lithotripters to measure at the focus [10] because it has several unique advantages compared to the other types of hydrophones: (i) The strong adhesion of water on the glass reduces nucleation of cavities the sensor, (ii) when the fiber tip is broken the fiber can easily be re-cleaved and cut, (iii) the entrainment of the fiber into a cavity leads to an instantaneous jump in the signal thus entrainment of the sensor is easily detectable, (iv) and, due to the well documented Gladstone-Dale relationship, calibration is a simple task.

Yet, there exist some difficulties with interpreting the signals. When studying waves with a steep pressure rise which are trailed by a negative pressure cycle very strong shot-to-shot variations are observed close to the moment when the negative pressure recovers. Averaging the signal over multiple events removes these oscillations but leaves the experimenter with some discomfort on how to interpret the data. An example of the signal which is achieved by this averaging procedure is depicted in Fig. 4.1.(thick line). The measurements were done in partially degassed Millipore (Milli-Q synthesis A10) water.

The contribution of cavitation on the waveform has been reported by Pishchalnikov et al. [11] They find a shortening of the tensile wave and explain it with the loss of energy from the tensile wave due to the growth of cavitation bubbles in the liquid. In recent simulations by Liebler et al. [12] the non-linear wave equation model was coupled with an effective medium which described the gas phase. They revealed that the waveform can be greatly altered: not only the tensile phase is shortened but also a second pressure increase following the shortened tensile phase is found in the simulations and backed convincingly with experiments. The altered waveform, which has also been reported by Arora et al. [13], is explained by Liebler et al. [12] by the presence of cavitation bubbles 


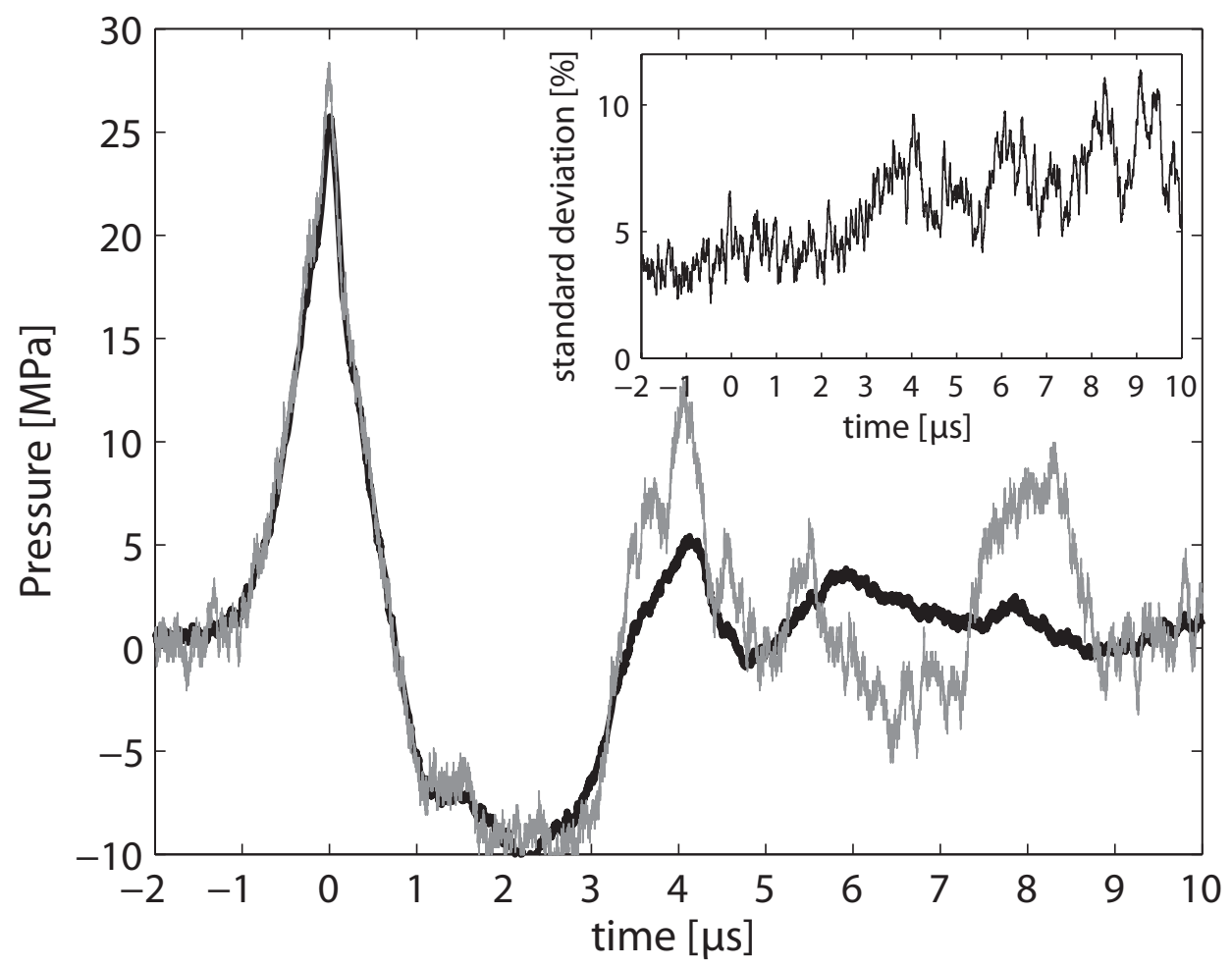

Figure 4.1: Pressure signals recorded with a FOPH device in degassed Millipore water. An averaged signal (black line) and a randomly selected single recording (gray line) are compared. The inset depicts the standard deviation (SD) of the signal as a function of time. The scale is given in percentage of the average peak pressure. Interestingly the standard deviation increases at time $t=3.2 \mu$ s that is when the pressure recovers.

disturbing the focusing of the diffracted waves from the transducer edge.

The averaged waveform over 18 pressure signals presented in Fig. 4.1 is compared with a randomly chosen recording from this set (thin line in Fig. 4.1). The variations between the single and the averaged signal remain small during the initial pressure rise to $26 \mathrm{MPa}$ and drop to $-10 \mathrm{MPa}$. This initial variability can be explained with the noise of the laser source and measurement noise of the photodetector. However, at time $\mathrm{t} \approx 3 \mu \mathrm{s}-$ that is, when the pressure recovers - clear differences appear. These are detailed in the inset of Fig. 4.1 by plotting the standard deviation of the signal.

What causes the loss of reproducibility in the data? Candidates are cavitation bubbles emitting pressure waves, yet we cannot exclude cavitation occurring on the glass fiber which might affect the light transmission in the glass fiber as was suggested by Pishchalnikov and colleagues [11]. We are now looking for 


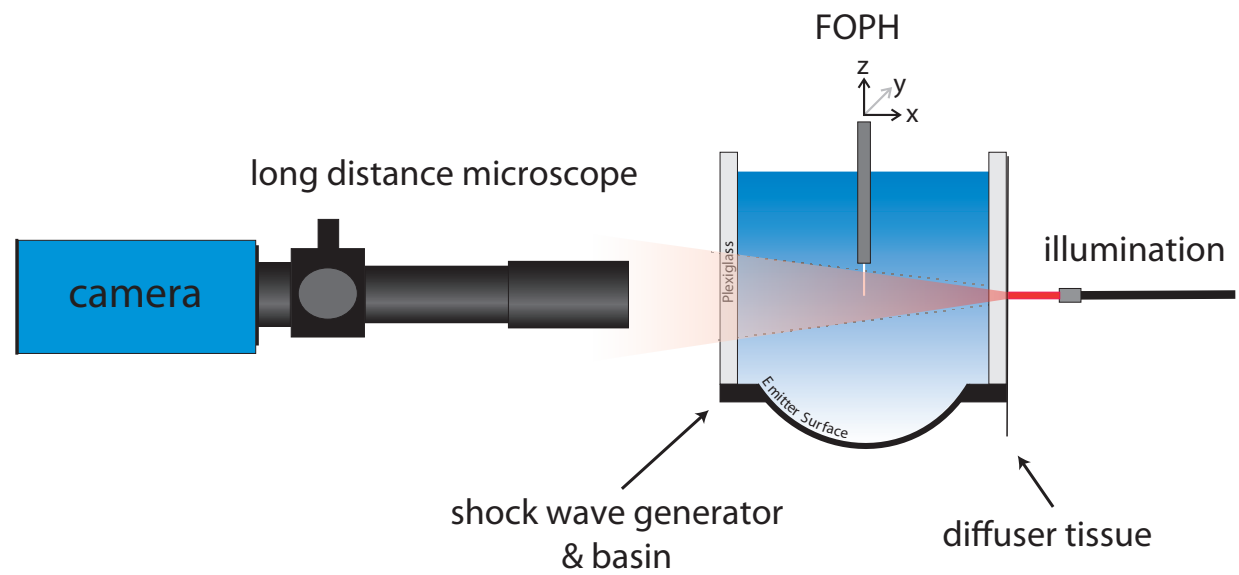

Figure 4.2: Experimental setup used both for pressure measurements and for shadowgraphy imaging.

a way to decrease cavitation activity only: In the work of Liebler et al. [12], it was reported that the pressure signal is affected by adding small amounts of a mild acid (acetic acid). Their explanation was: cavitation is reduced by chemically dissolving calcite particles in the water, a hypotheses put forward by Eisenmenger and Pecha [14].

Here, single flash imaging of the interaction of the wave with a fiber optic sensor is used to document the effect of cavitation on the glass fiber sensor. First we describe the experimental set-up which allows to conduct this task and visualize the interaction of the pressure-wave with the glass fiber tip.

\subsection{Experimental setup}

The pressure waves are generated with a piezoelectric device used for shock wave therapy (Piezolith 100, Richard Wolf GmbH, Knittlingen, Germany) [15]. The pressure measurements are done with the fiber optic hydrophone (FOPH 500, RP Acoustics, Stuttgart, Germany) which is positioned in the focus with a motorized three axis translation stage. The shock wave and the cavitation dynamics are visualized with a shadowgraphy technique using stroboscopic illumination (Fig. 4.2). The image of the shock wave and the cavitation bubbles are illuminated with a frequency doubled Nd:YAG laser pulse of 7 ns duration (Solo PIV, New Wave Research, wavelength $532 \mathrm{~nm}$ ) fed into a fluorescent cell filled with an ethanol-dye mixture $(0.417 \mathrm{mg} / \mathrm{ml}, L D S$ 698, Exciton Inc., Dayton, U.S.) and then coupled into a glass fiber to the shock wave setup located 
on a second table. The fluorescent light greatly reduces the coherent nature of the laser making it suitable as a light source. The light escapes the other fiber end and then becomes slightly diffused with a tissue paper.

The images are taken with a charge coupled device camera (Imager 3S, LaVision, Goettingen, Germany) which is equipped with a long distance microscope (K2, Infinity, U.S.) and a CF3 objective. The optical resolution is $1.52 \mu \mathrm{m} / \mathrm{pixel}$. The timing unit (BNC 555, Berkeley Nucleonics, CA, U.S.) which is triggered through an induction coil connected to the shock wave generator fires the laser and the camera at variable delays with respect to the shock wave. In contrast to prior high-speed framing studies on shock wave inception of cavitation, for example by Ohl [7], we emphasized on the quality of the pictures to resolve the shock waves and freeze the bubbles in stop motion. This was achieved with a single frame technique, i.e., the images are from different experimental runs with the time of camera shifted. The travel time of the wave from the transducer to the acoustic focus where the fiber optic probe hydrophone is located is approximately $50 \mu \mathrm{s}$.

\subsection{Results}

A stroboscopic picture sequence from the plain water experiment is shown in Fig. 4.3 and they are conducted in the same setup as the recording of the pressure signals shown in Fig. 4.1. The first frame displays the wave shortly after it passed over the glass fiber tip. The time $t=0$ is defined when it just meets with the glass fiber tip. Shadowgraphic imaging technique is sensible to the second derivative of the index of refraction [16]. Thus, the structure depicted in stop-motion in Figure 4.1 is the image of the pressure maximum of the wave, see $t=0$ in Figure 4.1. At $t=1.4 \mu$ s cavitation bubbles nucleate in the liquid. Spherical pressure waves appear shortly after, and reach about the position of the fiber tip in the fourth frame, $t=3.0 \mu \mathrm{s}$. This correlates with the start of the signal deviations in the pressure recordings. The spherical waves are created from collapsing cavitation bubbles. Presumably, the second pressure increase following the tensile phase causes the shrinkage of the bubbles. The origin of the pressure increase is likely to be a diffraction effect as is reported in Liebler et al. [12].

Let's now have a look on the cavity dynamics after the acetic acid (4 vol \%) has been added to the water. This concentration was chosen because Liebler et al. [12] observed excellent agreement of a bubble-free simulation and FOPH measurements. It was suggested, that cavitation activity is suppressed and the wave is not altered by the interaction with bubbles. Figure 4.4 depicts the shadowgraphy sequence in the solution with acetic acid. In the first two 


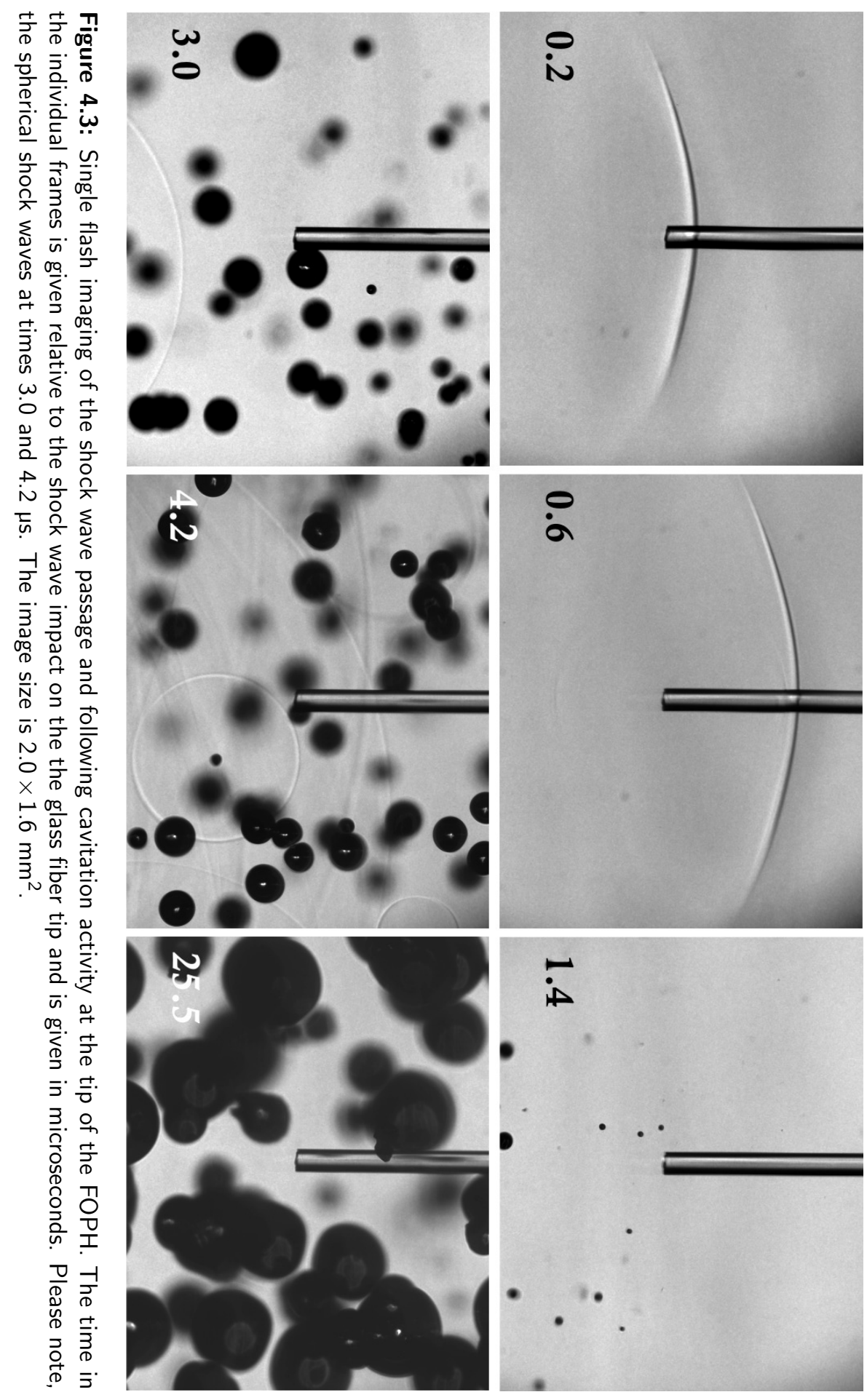



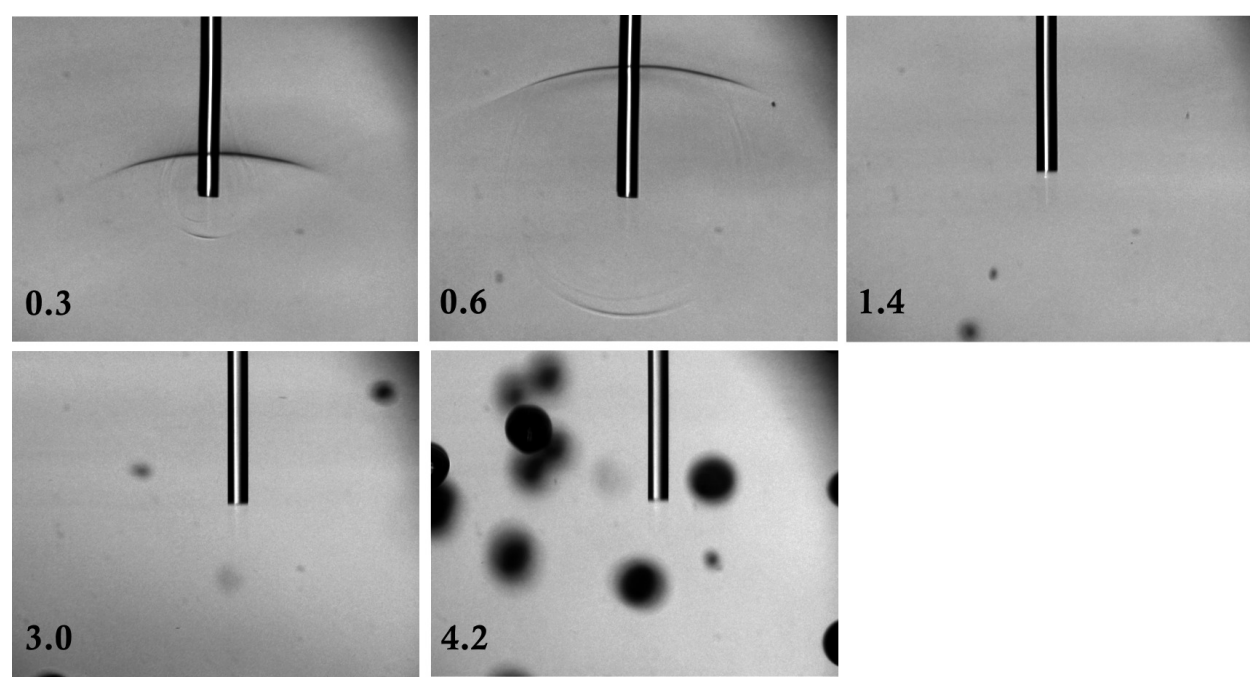

Figure 4.4: Flash photography of the shock wave passage and following cavitation activity at the tip of the FOPH in a water-acid solution ( $4 \%$ vol acetic acid). The individual times stated in the are with respect to figures are $0.2,0.6,1.4,3.0,4.2$, and $25.5 \mu$ s after the shock wave impact on the fiber tip.

frames the primary wave and also the reflected wave from the tip of the fiber is visualized. The initial pressure wave form is hardly altered as compared to the water case. The first cavities appear at $t=1.4 \mu$ s but only two, a tenth of the number of bubbles from the plain water case. A second difference is that at later times we don't find spherical waves emitted from collapsing cavitation bubbles. This is interesting, because a few bubbles are nucleated in the field of view and thus might qualify for a forced collapse. The addition of acid to the liquid causes a strong reduction on the number of bubbles. But it also causes a second effect, it changes the time averaged pressure recordings. We find in the plain water case a second overpressure peak after the pressure recovered from the negative pressure, $\mathrm{t}=4.1 \mu \mathrm{s}$ in Figure 4.1 which is absent here, very similar to the findings of Liebler et al. [12].

\subsection{Discussion \& conclusion}

Presumably, the second overpressure is a nonlinear effect of the diffraction wave propagation in the bubbly/compressible liquid. By adding acid much less bubbles are created and the diffraction wave becomes unaffected. Therefore, the remaining cavities are not compressed and do not cause secondary shock waves, which then in return leads to a more reproducible signal. This finding is 


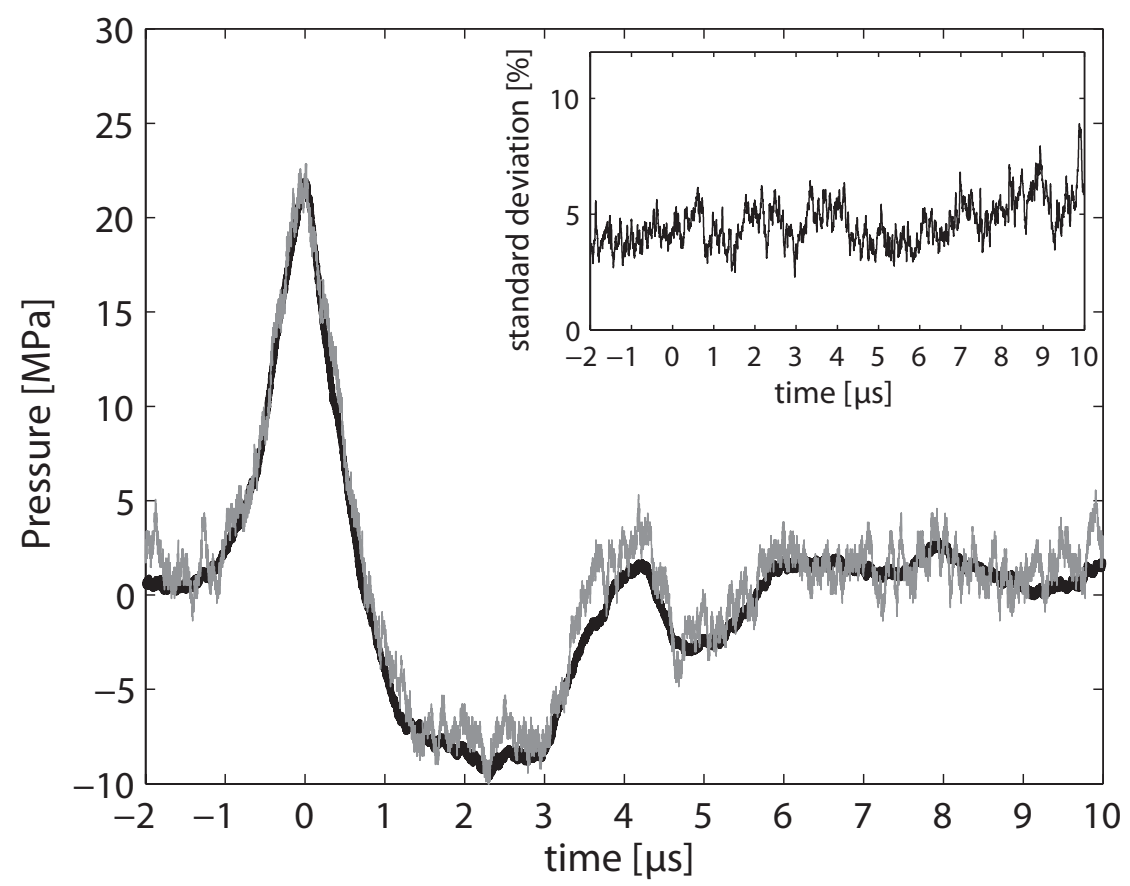

Figure 4.5: Pressure signals recorded with a FOPH device in degassed Millipore water with $4 \%$ vol. acetic acid. An averaged signal (black line) and a randomly selected single recording (gray line) are compared. The inset depicts the standard deviation (SD) of the signal as a function of time. Here, the SD stays constant for the measured time. The positive peak pressure recorded with the FOPH is slightly less than shown in Fig 4.1 which we explain with some spatial shift of the fiber tip of less than $100 \mu \mathrm{m}$ during the addition and stirring of the liquid.

strengthened by the plot of the standard deviation in Figure 4.5. It stays constant over the measured time. Our finding is that the pressure signal obtained in a cavitation liquid consists of a superposition of the pressure induced by the direct wave and from secondary waves emitted by collapsing bubbles. We extend the results from Liebler et al.[12] that not only the waveform is modified by its passage through the bubbly liquid. Additionally, the forced collapse and the thereby emitted secondary pressure and shock waves are picked up by a fiber optic probe hydrophone. The spatial randomness of the cavitation events leads to the noise-like signal registered when the pressure recovers from the tensile phase. 


\section{Bibliography}

[1] M. Bailey, V. Khokhlova, O. Sapozhnikov, S. Kargl, and L. Crum. Physical mechanisms of the therapeutic effect of ultrasound (a review). Acoustical Physics, 49:369-388, 2003.

[2] O. A. Sapozhnikov, A. D. Maxwell, B. MacConaghy, and M. R. Bailey. A mechanistic analysis of stone fracture in lithotripsy. J. Acoust. Soc. Am., 121(2):1190-1202, 2007.

[3] Warden S.J. A new direction for ultrasound therapy in sports medicine. Sports Medicine, 33:95-107(13), 2003.

[4] Zhen X., A. Ludomirsky, L.Y. Eun, T. L. Hall, B. C. Tran, J. B. Fowlkes, and C. A. Cain. Controlled ultrasound tissue erosion. Ultrasonics, Ferroelectrics and Frequency Control, IEEE Transactions on, 51(6):726 -736, 2004.

[5] J. E. Kennedy, G. R. ter Haar, and D Cranston. High intensity focused ultrasound: surgery of the future? Br. J. Radiol, 76(909):590-599, 2003. doi: $10.1259 / \mathrm{bjr} / 17150274$.

[6] C. E. Brennen. Cavitation and bubble dynamics. Oxford University Press, New York, 1995. ISBN 0195094093.

[7] C. D. Ohl. Cavitation inception following shock wave passage. Physics of Fluids, 14(10):3512-3521, 2002.

[8] J. Staudenraus and W. Eisenmenger. Fibre-optic probe hydrophone for ultrasonic and shock-wave measurements in water. Ultrasonics, 31(4):267 - 273, 1993.

[9] J. F. Krücker, A. Eisenberg, M. Krix, R. Lötsch, M. Pessel, and H. G. Trier. Rigid piston approximation for computing the transfer function and angular response of a fiber-optic hydrophone. J. Acoust. Soc. Am., 107 (4):1994-2003, 2000.

[10] International Electrotechnical Committee. Ultrasonics - pressure pulse lithotripters - characteristics of fields, IEC Standard, 1998.

[11] Y. A. Pishchalnikov, O. A. Sapozhnikov, M. R. Bailey, I. V. Pishchalnikova, Jr. J. C. Williams, and J. A. McAteer. Cavitation selectively reduces the negative-pressure phase of lithotripter shock pulses. Acoustics Research Letters Online, 6(4):280-286, 2005. 
[12] M. Liebler, T. Dreyer, and R. E. Riedlinger. Nonlinear modeling of interactions between ultrasound propagation and cavitation bubbles. Acta Acustica united with Acustica, 92:165-167(3), 2006.

[13] M. Arora, C. D. Ohl, and D. Lohse. Effect of nuclei concentration on cavitation cluster dynamics. J. Acoust. Soc. Am., 121(6):3432-3436, 2007.

[14] W. Eisenmenger and R. Pecha. New Species of Cavitation Nuclei. Fortschritte der Akustik, DAGA03, page 842843, 2003.

[15] N. Bremond, M. Arora, C. D. Ohl, and D. Lohse. Controlled multibubble surface cavitation. Phys. Rev. Lett., 96(22):224501, 2006.

[16] G. S. Settles. Schlieren and shadowgraph techniques: Visualizing phenomena in transparent media. Experimental Fluid Mechanics. Springer-Verlag, 2001. 


\section{5}

\section{Acoustically induced bubble pinch-off}

The acoustic nucleation of pockets of gas entrapped in cavitation nuclei has been well studied and described for a single pressure pulse. Here we explore the rich behavior displayed when pre-defined cavitation nuclei are driven continuously in the $\mathrm{kHz}$ regime ( 80 and $200 \mathrm{kHz}$ ). The nuclei consist of stabilized gaspockets in cylindrical micropits etched in silicon substrates. It is found that above an acoustic pressure threshold the behavior of the liquid-gas meniscus switches from the well known stable drum-like vibration to bubble expansion and deformation, frequently resulting in microbubble pinch-off. Just above this threshold small bubbles are continuously and intermittently ejected. At elevated acoustic pressures the pinch-off mechanism becomes more pronounced and cavitation bubble clouds are formed which remain in the vicinity of the micropit bubble. Surprisingly, the resulting loss of gas typically does not lead to the deactivation of the pit which can be explained by gas diffusion processes.

\subsection{Introduction}

Most applications based on acoustic cavitation, such as for example ultrasonic cleaners, emulsifiers or sonochemical reactors rely on the spontaneous and seemingly random appearance of cavitation bubbles. Also the subsequently generated cavitation state, meaning the bubble sizes, locations, dynamics and so on is extremely difficult to describe and even more difficult to prescribe. This is mainly due to the inherent chaotic nature of cavitation and its complex dependence on the liquid and acoustic parameters. Manufacturers and researchers therefore have to resort to empirical methods to optimize the desired mechanical or chemical effects induced by acoustic cavitation bubbles [1-3]. The resultant of these effects is not only determined by the magnitude of the response of each individual bubble, but also by the total number of bubbles and their locations. The increase of cavitation activity would seem just a matter of increasing 
the acoustic amplitude, however, due to effects such as acoustic shielding and bubble-bubble interactions, this does not necessarily lead to an increase in efficiency. On the other hand, there is also a frequency dependent lower threshold for cavitation activity, usually referred to as the nucleation threshold [4]. Hence in the situations where lower acoustic amplitudes are required, e.g. to prevent heating or surface damage [5], it might not even be possible to generate or sustain a sufficient bubble population.

Evidently, enhancing cavitation bubble mediated effects may be achieved by actively regulating not only the acoustic amplitude but also the bubble population. Therefore, an ideal solution is to introduce microbubbles of the required size into the liquid at the required locations. For example, various studies have shown that the presence of ultrasound contrast agents - microbubbles stabilized by an external shell - significantly enhances cavitation related effects [6, 7]. Other methods such as bubble injection using gas flow through membranes [8] or flow focusing devices [9] might also be beneficial for increasing bubble populations in acoustic cavitation.

Here we present results of a different method to locally enhance acoustic cavitation activity in a continuous ultrasound pressure field. Our method consists of introducing microbubbles into the liquid which are entrapped and stabilized in micromachined, well defined cylindrical cavities, an example of which is shown in Figure 5.1. These bubbles are essentially artificial versions of naturally occurring cavitation nuclei better known as "crevice bubbles" [10, 11]. The use of artificial crevice bubbles was motivated by earlier studies of Bremond et al. [12, 13] and Borkent et al. [14] where the response of the entrapped bubble to a single shock wave was investigated. In this chapter we investigate the dynamics of stabilized cylindrical micropit bubbles under continuous ultrasound irradiation. It will be shown that the presence of stabilized microbubbles significantly enhances local cavitation activity by bubble pinch-off processes.

Unlike a spherical bubble which will quickly dissolve under the excess pressure due to surface tension, an entrapped bubble can be both in stable mechanicaland diffusive equilibrium for an extended period of time. The presence of a crevice bubble nucleus basically constitutes a "weak-spot" in the liquid. Any change in the macroscopic conditions will cause a volumetric response of the bubble as it seeks to reestablish its equilibrium state. Nucleation is the event where the equilibrium cannot be established and the bubble expands out of the micropit [11, 14]. There are three different but interrelated ways for this to occur. Nucleation can be realized by pressure reduction, but also by an increase in gas saturation or by a rise in temperature. Bubble formation from crevice bubbles is essential, not only in cavitation inception but also in supersaturated liquids such as carbonated beverages [15, 16] and in liquid pool boiling [17]. 


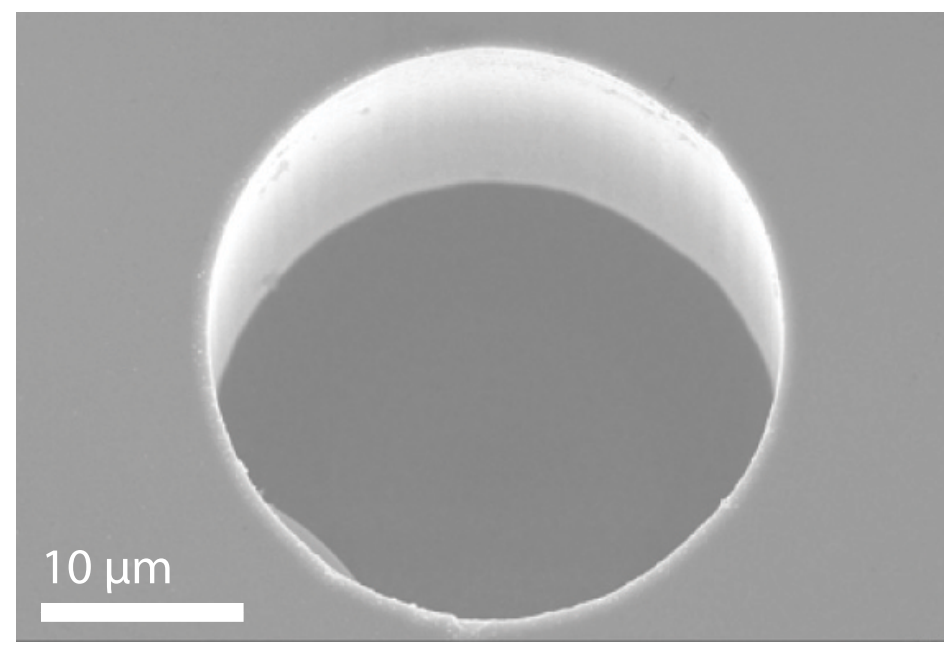

Figure 5.1: Micromachined cylindrical cavity of $30 \mu \mathrm{m}$ diameter and $10 \mu \mathrm{m}$ depth in a silicon substrate.

It is important to make a distinction between nucleation, i.e. the unstable growth of the bubble outside the pit, and a subsequent possible bubble pinchoff from the nucleus. Many authors use the term nucleation for the combined process, however the underlying mechanisms are different. Once nucleated, a bubble can detach from the micropit due to effects such as buoyancy, liquid drag or acoustic pressure gradients. Bubble detachment, or pinch-off is a well studied effect in nucleate boiling [18] and particularly in supersaturated liquids [16], but surprisingly not in acoustic cavitation. Even though bubble detachment from entrapped surface bubbles is normally assumed to occur in acoustic cavitation, to the authors knowledge, there exists only one study in which this is actually reported. In 1962 Hughes \& Nyborg [19] drilled holes of $200 \mu \mathrm{m}$ in a sonotrode tip in order to enhance cavitation effects at reduced acoustic amplitudes. They observed bubble growth, surface instabilities and bubbles leaving the holes but remained rather speculative on the details of the process. Their method however did result in increased cavitation activity due to the presence of artificial crevice bubbles.

Here we are interested in the response of artificial micropit bubbles to a continuous acoustic field and how this response can contribute to enhancement of the local cavitation activity. In the following, the process of bubble nucleation and pinch-off in acoustic cavitation is investigated in more detail using highspeed imaging methods. 


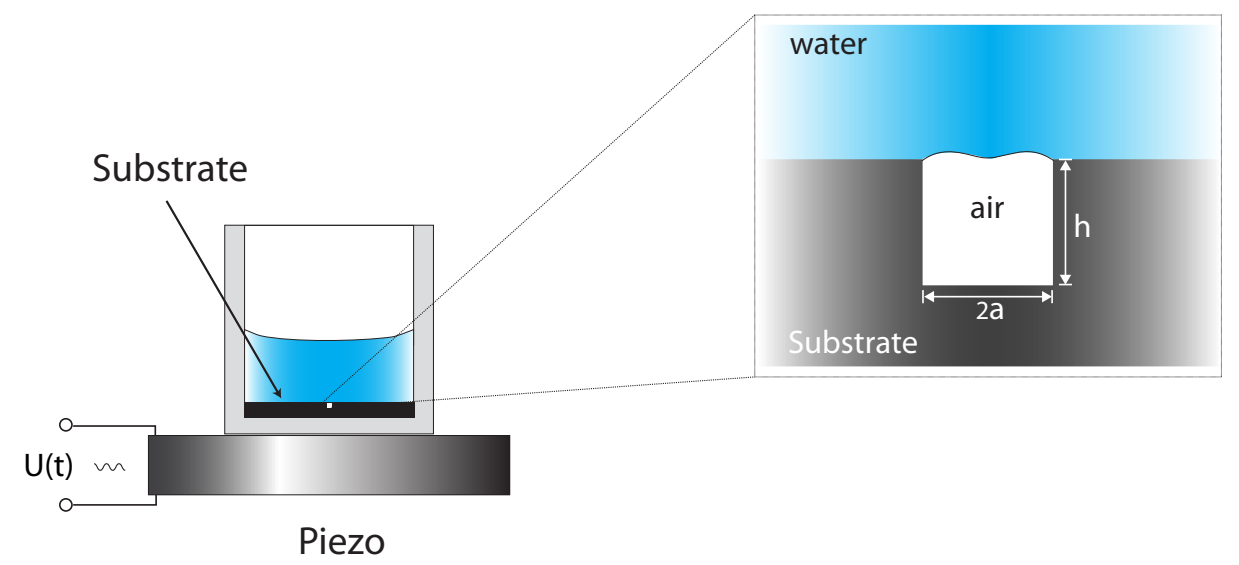

Figure 5.2: Schematic of the piezo-cuvette combination in side view.

\subsection{Materials \& methods}

\subsubsection{Silicon micromachining}

In order to obtain micropit bubbles which are comparable in size with naturally occurring crevice bubbles, but large enough to allow visual observations we chose to create cylindrical pits of $30 \mu \mathrm{m}$ in diameter and similar depth. The pits were micromachined under clean room conditions in double-side polished silicon wafers. The wafer was spin coated with the photosensitive substance (Olin 12), on which the designed pattern was transferred with a mask aligner EV620 (photolithography). After development the holes were etched into the silicon substrate at the desired depth using a plasma dry-etching machine (Adixen AMS 100 SE Alcatel process BHARS). The wafer was diced into square pieces of $1 \times 1 \mathrm{~cm}^{2}$. We found that a stable liquid-gas micromeniscus was always present after immersion of the substrate even though no extra measures were taken to hydrophobize the micropit as was done in the previous studies by e.g. Bremond et al [12, 13].

\subsubsection{The acoustic field}

For stability and convenient optical access, the substrate was placed on the bottom of a small container (optical quality glass, inner dimensions $1 \times 1 \times$ $1 \mathrm{~cm}^{3}$, or a second type, inner diameter $1.5 \mathrm{~cm}$, depth $3 \mathrm{~mm}$ ) to which a piezoelectric element (Ferroperm PZ27 $6 \mathrm{~mm}$ thickness and diameter of 2.5 $\mathrm{cm}$ ) was glued (see Figure 5.2). The maximum response of the piezo and 


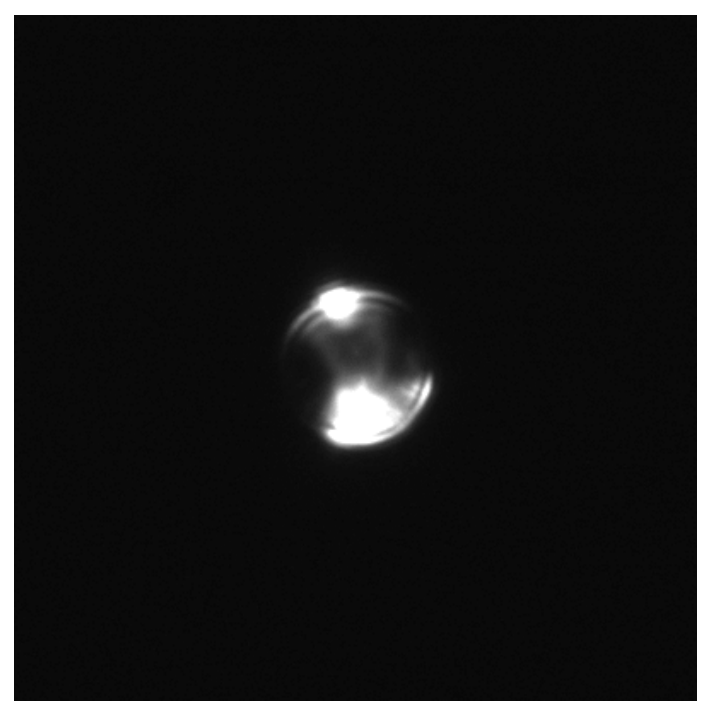

Figure 5.3: Top view image of a $30 \mu \mathrm{m}$ micropit obtained with dark-field illumination. The scattered light is an indication of the presence of the liquid-gas micromeniscus.

cuvette combination was found to occur at frequencies close to $80 \mathrm{kHz}$ and $200 \mathrm{kHz}$ corresponding to radial and thickness vibration modes. Upon adding demineralized water (left standing for one day to ensure full saturation) a pocket of air was entrapped within the cylindrical micropit. The presence of the liquidgas interface could easily be verified using dark-field illumination microscopy (e.g. see Figure 5.3).

Since the applied acoustic field is continuous, a standing wave is formed depending on the height of the liquid. It was found that the largest response of the micropit bubble occurred for liquid heights close to one-quarter or threequarters of the acoustic wavelength. This situation corresponds to a pressure anti node situated on the substrate and a pressure node at the free water-air interface. Water heights close to $\frac{\lambda}{4}=4.7 \mathrm{~mm}$ for $80 \mathrm{kHz}$ and $\frac{3 \lambda}{4}=5.6 \mathrm{~mm}$ for $200 \mathrm{kHz}$ were used,

The piezoelectric element was actuated using a sinusoidal voltage generated by a function generator (Tabor Electronics Ltd., model WW1072) which was amplified using a wide band amplifier (Krohn-Hite, model 7602). The input voltage and current signal were measured using an oscilloscope (Tektronix, DPO 4043) and a current probe (Tektronix, CTA-2). The small liquid volume and the close proximity to the piezo element hampered reliable pressure measurements therefore the input power was taken as a relative measure. 


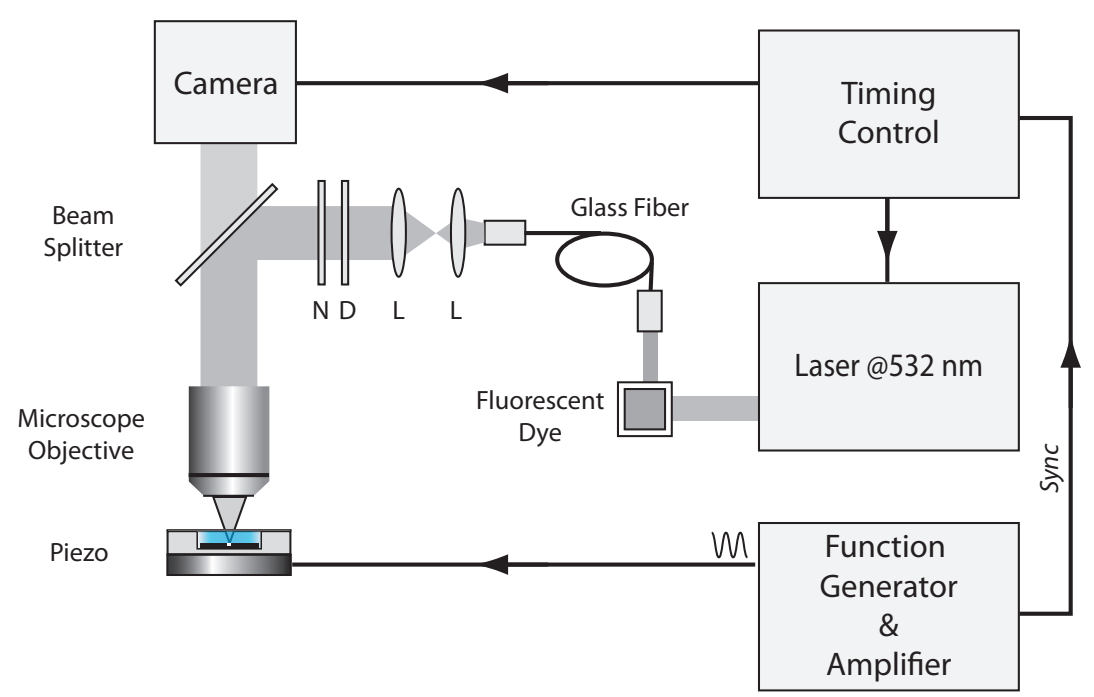

Figure 5.4: Schematic overview of the setup used for single flash imaging of micropit bubble pinch-off and cavitation in top-view. After exiting the fiber the beam is diffused (D) and filtered from any remaining laser light using a notch-filter (N) (Semrock, NF01-532U-25). Illumination of the full field of view is achieved by adjusting the angle of divergence of the beam using two positive lenses $(L)$.

\subsubsection{Imaging methods}

The timescale of acoustic cavitation is set by the period of the driving acoustic field. Hence, in order to resolve the dynamics of ultrasonically driven (micropit) bubbles, high-speed imaging techniques are required. Here we use two types of high-speed cameras which are the Photron, model SA1.1 with framerates up to $500 \mathrm{kfps}$ or the Brandaris 128 capable of framerates up to $25 \mathrm{Mfps}$ [20]. These cameras are used to capture transient effects. High-speed cameras allow visualization of extremely fast events, however the limited spatial resolution of the produced images complicates image analysis. Therefore an additional ultrashort single flash imaging method was applied which enabled recording of high resolution images without motion induced blur. The details of this method are described in chapter 3 of this thesis. The setup used for this particular method is depicted in Figure 5.4. Here the fluorescent laser dye dissolved to saturation in ethanol (LDS 698, Exciton Inc., Dayton, U.S.) was contained in a quartz cuvette (Hellma Quartz Suprasil $10 \times 10 \times 40 \mathrm{~mm}^{3}$ ). The geometry of the cuvette combined with the concentrated dye solution results in high-intensity fluorescence emerging at 90 degrees with the exciting laser beam [21]. 

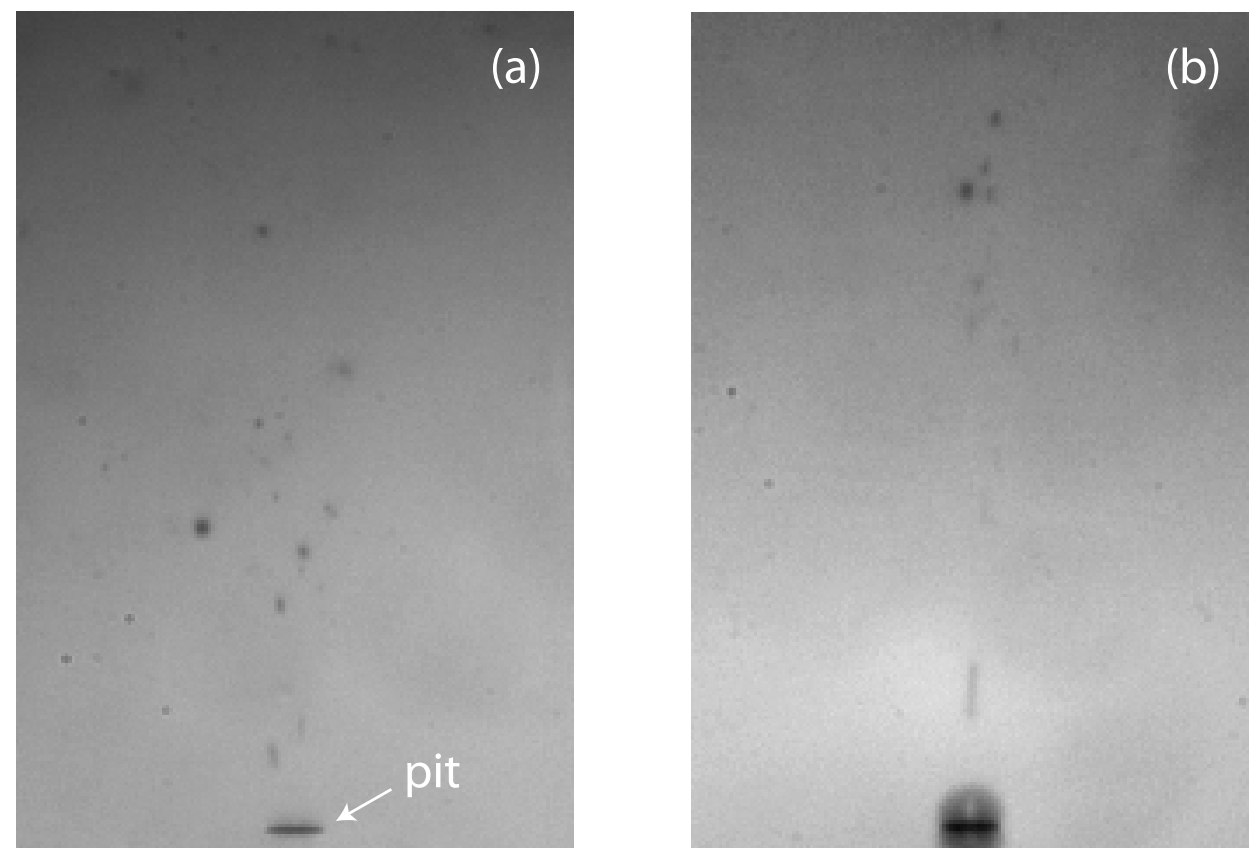

Figure 5.5: Selected frames of a micropit bubble expelling microbubbles. The left image (a) shows irregular bubble pinch-off where microbubbles move upward in various angles with the vertical axis. The right image (b) shows a regime where the micropit bubble extends outside the pit perimeter and the pinched off bubbles move upwards along the vertical axis.

The fluorescent illumination can be used in back-lighting configuration for side view or, as shown in Figure 5.4 in reflective mode (top view) enabled by the reflectiveness of the silicon substrate. For this purpose the light pulse is relayed using a glass fiber and coupled into the microscope (Olympus). Image magnification was obtained with a long working distance objective (LMPLFLN 10×, Olympus, WD $21 \mathrm{~mm} / \mathrm{NA}=0.25$ or the SMPLFLN 20×, Olympus, WD 25 $\mathrm{mm} / \mathrm{NA}=0.25)$. The illumination flash was carefully synchronized with the single frame camera (Lumenera LM165, pixel size of $6.45 \times 6.45 \mu \mathrm{m}^{2}$, resolution $1392 \times 1040)$ using a pulse delay controller (Berkeley Nucleonics, model 575). Additionally, the delay controller was synchronized with the acoustic driving signal. 


\subsection{Results}

\subsubsection{Driving at $80 \mathrm{kHz}$}

First we consider the micropit bubble driven by an $80 \mathrm{kHz}$ standing acoustic field which is below its first linear resonance of approximately $98 \mathrm{kHz}$ based on the theoretical description presented in chapter 2. For small driving amplitude there was no visible response of the micropit bubble. For increased acoustic pressures however streams of microbubbles of various sizes could be observed which were ejected intermittently from the micropit bubble. This behavior was found to be reproducible, nevertheless the character of the bubble ejection mechanism could be one of two different types, both occurring under similar conditions. For the first type the liquid-gas interface did not extend visibly outside the pit while bursts of microbubbles were expelled upwards, seemingly originating from different locations on the interface (Figure 5.5(a)). The details of the pinch-off in this regime could not be resolved optically. This in contrast with the second type of pinch-off, where the micropit bubble expands hemispherically outside the micropit during the low pressure phase of the acoustic field and withdraws again inside the pit when the pressure recovers.

A sequence of frames of a high-speed movie of a bubble pinch-off event in this regime is shown in Figure 5.6. Here a microbubble of approximately $5 \mu \mathrm{m}$ in diameter is pinched-off during the collapse phase of the micromeniscus somewhere between $20 \mu \mathrm{s}$ and $22 \mu \mathrm{s}$. Though difficult to distinguish, the interface of the micromeniscus changes from its initial hemispherical shape to a bell-shape during the collapse phase. This indicates that the side wall of the meniscus is collapsing before the top wall. Occasionally this radial flow focusing is sufficient to overtake the top part of the interface thereby separating it from the rest of the meniscus. In all of the observed pinch-off events of this type, the bubble separates from the meniscus during the contraction phase, most likely corresponding to the high pressure phase of the acoustic cycle. The resulting stream of microbubbles is visible in Figure 5.5(b). Because of the axial symmetric character of the pinch-off also the trajectories of the bubbles are mainly along the vertical axis.

What causes these two types of bubble pinch-off? From the observations we may speculate that the bubbles are generated by a "folding" of the liquid-gas interface resulting from large amplitude capillary waves. These closed capillary waves resemble the famous "Crapper wave" solutions [22]. The regime shown in Figure 5.5 (a) where bubbles are pinched-off from different locations on the interface would then correspond to a shorter capillary wavelengths compared to the regime shown in Figure 5.5 (b) and Figure 5.6 

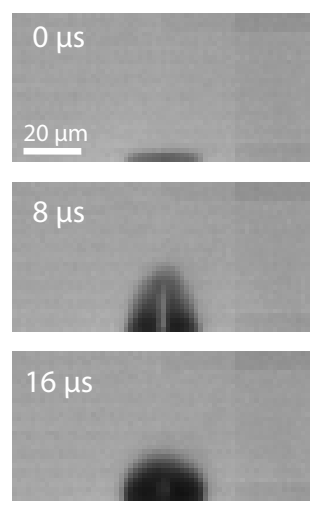

$24 \mu \mathrm{s}$

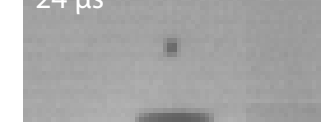

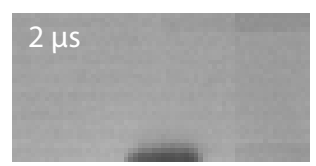
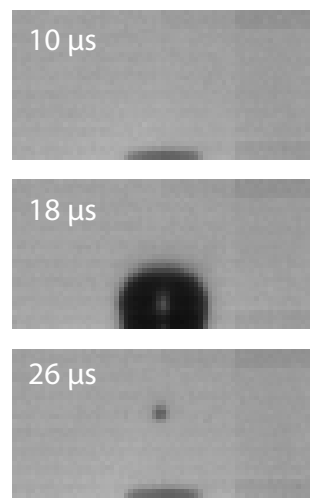
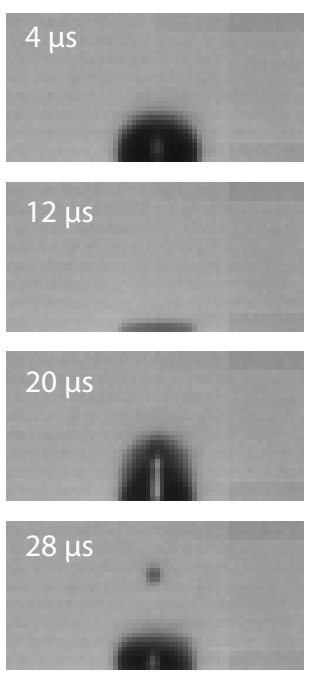
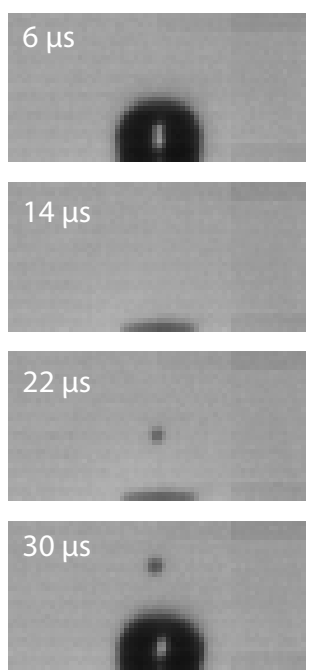

Figure 5.6: Sequence of frames from a $500 \mathrm{kfps}$ high-speed movie showing the response of the micropit bubble during 2.5 acoustic cycles. In the second cycle from 12 to 24 $\mu \mathrm{s}$ a $5 \mu \mathrm{m}$ diameter microbubble is pinched-off.

Estimates of the bubble sizes and pinch-off rates were obtained using image

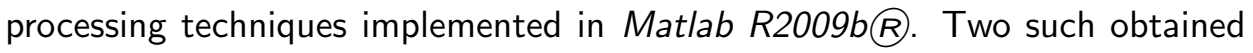
time traces are shown in Figure 5.7, one for the irregular type pinch-off (a) and one for the regular type pinch-off (b). These traces reveal that the bubble pinch-off processes are quite erratic, both in pinch-off rate, as well as the ejected bubble sizes. At some instances (e.g. near $60 \mathrm{~ms}$ in Figure 5.7(a)) bursts of multiple bubbles (typically with radii less than $5 \mu \mathrm{m}$ ) are expelled while at other moments it can take several milliseconds before the next pinchoff event takes place. The average rate of bubble pinch-off for these particular traces was approximately $2 \mathrm{kHz}$ or once every 40 acoustic cycles. The bubble size distributions (shown in the insets in (a) and (b)) reveal that the majority of the ejected bubbles had radii less than $7 \mu \mathrm{m}$ where the larger bubbles typically resulted from bubble coalescence. Surprisingly, the irregular type pinch-off process appears to result in bubbles having radii mostly near $2 \mu \mathrm{m}$ while the regular type shows a broader distribution. Bubble coalescence was however more prevalent in the first type giving rise to larger bubble radii compared to that of the second type which is also reflected in the bubble distributions. It should be noted that smaller bubbles $(<1.5 \mu \mathrm{m}$ radius $)$ also were observed but limited image resolution prohibited estimation of their radii.

All of the observed ejected bubbles have radii smaller than the resonance radius of $38 \mu \mathrm{m}$ (equation 1.2) for this frequency. The size of the micropit bubble 

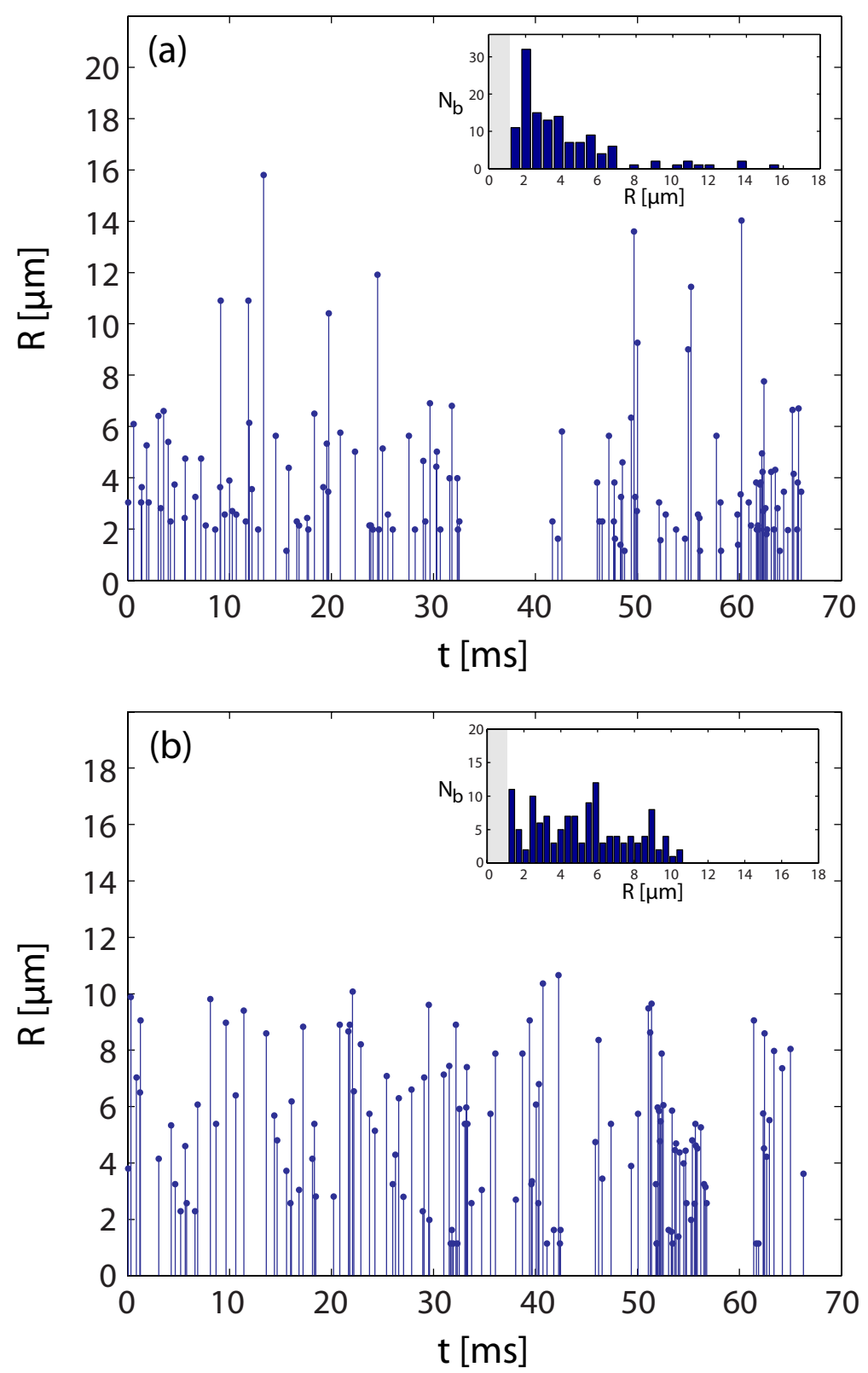

Figure 5.7: Ejected bubble events marked by bubble radius in time for the irregular type pinch-off (a) and regular type pinch-off (b). The insets show the histograms with $\mathrm{N}_{\mathrm{b}}$ the number of bubbles. The data was obtained from $15 \mathrm{kfps}$ high-speed movie recordings. The image resolution did not allow bubble sizing for radii smaller than $1.3 \mu \mathrm{m}$ (indicated by the gray areas in the distributions). 
on the other hand is closer to its linear resonance radius for this frequency (98 $\mathrm{kHz}$, see chapter 2) which may explain the difference in oscillation amplitude. For example, from the Rayleigh-Plesset equation (1.1) it follows that a spherical bubble with an equilibrium radius of $3 \mu \mathrm{m}$ would expand to a maximum radius of $4 \mu \mathrm{m}$ for an acoustic amplitude of approximately $90 \mathrm{kPa}$. Since such bubble expansions are not observed it follows that this pressure value is a conservative estimate for the lower driving amplitude required for bubble pinch-off as shown in Figures 5.5 and 5.6 .

From the recordings, estimates for the bubble velocities could be obtained. For the regular type it was found that the velocity of the bubbles immediately after pinch-off was typically as high as $2 \mathrm{~m} / \mathrm{s}$ and slowing down to about $50 \mathrm{~mm} / \mathrm{s}$ some $200 \mu \mathrm{m}$ above the substrate. For the irregular type pinch-off the velocities were found to be an order of magnitude lower. The larger number of bubble coalescence events observed in this regime compared to the regular type may be related to these lower velocities.

The most remarkable observation however was that even though the micropit bubble continuously ejected microbubbles, the loss of gas did not result in diminishing or deactivation of the process. The pinch-off rate corresponds to a gas volume flux in the order of $100 \mu \mathrm{m}^{3} / \mathrm{s}$ away from the micropit which must be balanced by an influx of equal magnitude. This transport is most likely of diffusive nature, where a rectifying mechanism may take place, similar to the well known process of rectified diffusion of spherical bubbles [23]. Here, both the area effect and the shell effect may occur. In addition, it is possible that the oscillation-induced microstreaming (see e.g. Miller [24]) draws in liquid from the bulk where the concentration of dissolved air is larger, thereby sustaining the pinch-off process.

\subsubsection{Driving at $200 \mathrm{kHz}$}

We now consider the response of the micropit bubble driven at $200 \mathrm{kHz}$. This frequency is approximately twice the linear resonance frequency $(98 \mathrm{kHz})$ and lies below the second resonance of $243 \mathrm{kHz}$. Similar to what was observed for the $80 \mathrm{kHz}$ driven micropit bubble, there is a threshold for the pressure above which bubble pinch-off occurs. Interestingly, in the regime below this threshold the liquid-gas interface exhibits alternating dark and bright rings which appear to move radially outward periodically with the driving frequency (see Figure 5.8(a)). Since it is difficult to reconstruct the shape of the interface based on the intensity of the reflected illumination it cannot be concluded a priori that these rings are due to surface ripples as described in chapter 2. Nevertheless, from these images it can be inferred that the interface retains its axial symmetry 

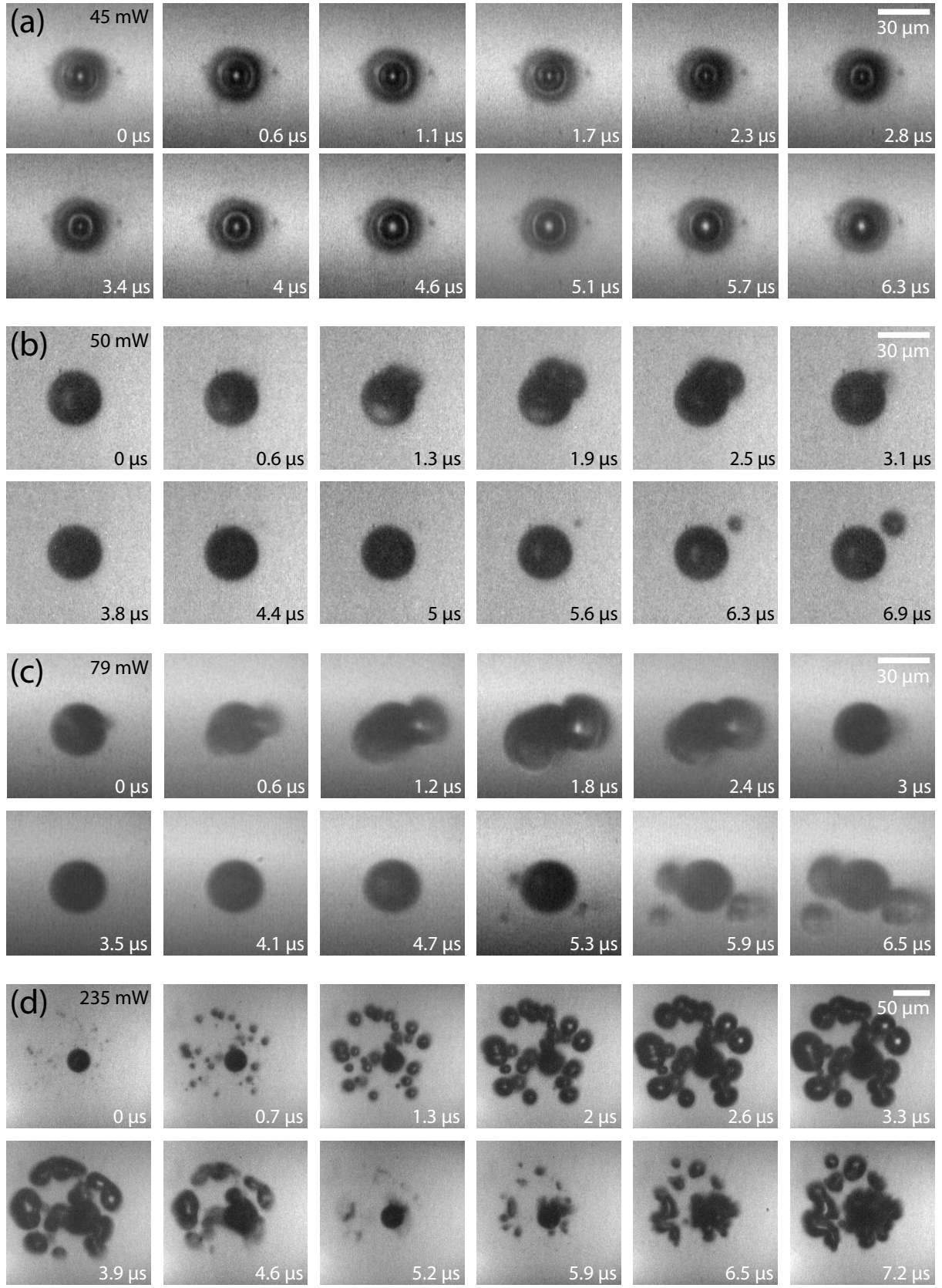

Figure 5.8: Four sequences of frames taken from high-speed recordings in reflective imaging mode (top-view) of the micropit for increasing driving amplitude. Sequence (a) is at approximately $45 \mathrm{~mW}$ showing ring-structures. Increasing the driving power to $50 \mathrm{~mW}$ induces interface deformation and pinch-off (b). Further increase $(79 \mathrm{~mW}$ in (c) and $235 \mathrm{~mW}$ in (d)) both increases the bubble number and maximum expansion. Note the different scale in (d). 
and oscillates at the same frequency as the driving acoustic field. This symmetry is lost however when the threshold is exceeded. Shown in Figure 5.8(b) is a sequence of frames taken from a high-speed movie where the driving pressure is slightly above the threshold, corresponding to an input power of $50 \mathrm{~mW}$. Here the rings are no longer visible and occasionally bubbles are pinched off which in turn respond to the acoustic field by expansion and collapse. In this regime the bubbles which are pinched-off generally are drawn back into the micropit bubble within at most 3 acoustic cycles, most likely due to mutual acoustic forces. In contrast to the $80 \mathrm{kHz}$ driven micropit bubble, the ejected microbubbles do not move upward but remain in close proximity of the substrate. From many similar recordings in this regime it appears that once a bubble is detached from the micropit bubble, a subsequent pinch-off event will not occur as long as the bubble is not recaptured. This may indicate a possible volume dependence. As shown in Figure 5.8(c), increasing the amplitude enhances the deformation of the interface and bubbles are continuously pinched-off from the liquid gas interface. Also the number of free bubbles oscillating just outside the pit perimeter increases as well as their maximum expansion. The bubbles still remain close to the micropit bubble and are observed to oscillate up to 3 or 4 cycles before fracturing or coalescing with the micropit bubble or other bubbles.

The same trend is followed for larger driving amplitudes; more bubbles are pinched-off expanding to larger volumes with dimensions exceeding the diameter of the micropit. As illustrated in Figure 5.8(d) a cavitation bubble cloud is formed which is centered on the micropit. Here both bubble deformation, coalescence and fission are more pronounced compared to lower driving conditions (Figure 5.8(b) and (c)). Due to these effects, the bubble population, meaning bubble locations and sizes, changes with every acoustic cycle. The response of an individual bubble in the cloud can therefore only be distinguished and followed in time during part of the expansion phase of the cycle (e.g. from 0.7 $\mu \mathrm{s}$ to $3.3 \mu \mathrm{s})$.

The increase in the number of bubbles with increasing driving amplitude is clearly shown in the graph of Figure 5.9. The estimates for the number of bubbles were obtained from high-speed recordings at different driving amplitudes. The number of bubbles varies considerably with each acoustic cycle (e.g. compare Figure $5.8(\mathrm{~d})$ at $\mathrm{t}=0.7 \mu \mathrm{s}$ and $\mathrm{t}=6.5 \mu \mathrm{s})$ resulting in a large variance. Exact bubble counts were difficult to obtain due to limited resolution as well as bubbles which remained undetected when positioned just above the micropit bubble or in contact with other bubbles.

The growth of the bubble cloud with increasing amplitude is illustrated in Figure 5.10. The frames in (a) show the scattered light from the bubbles in dark-field illumination mode giving an indication of the extent of the cloud. 


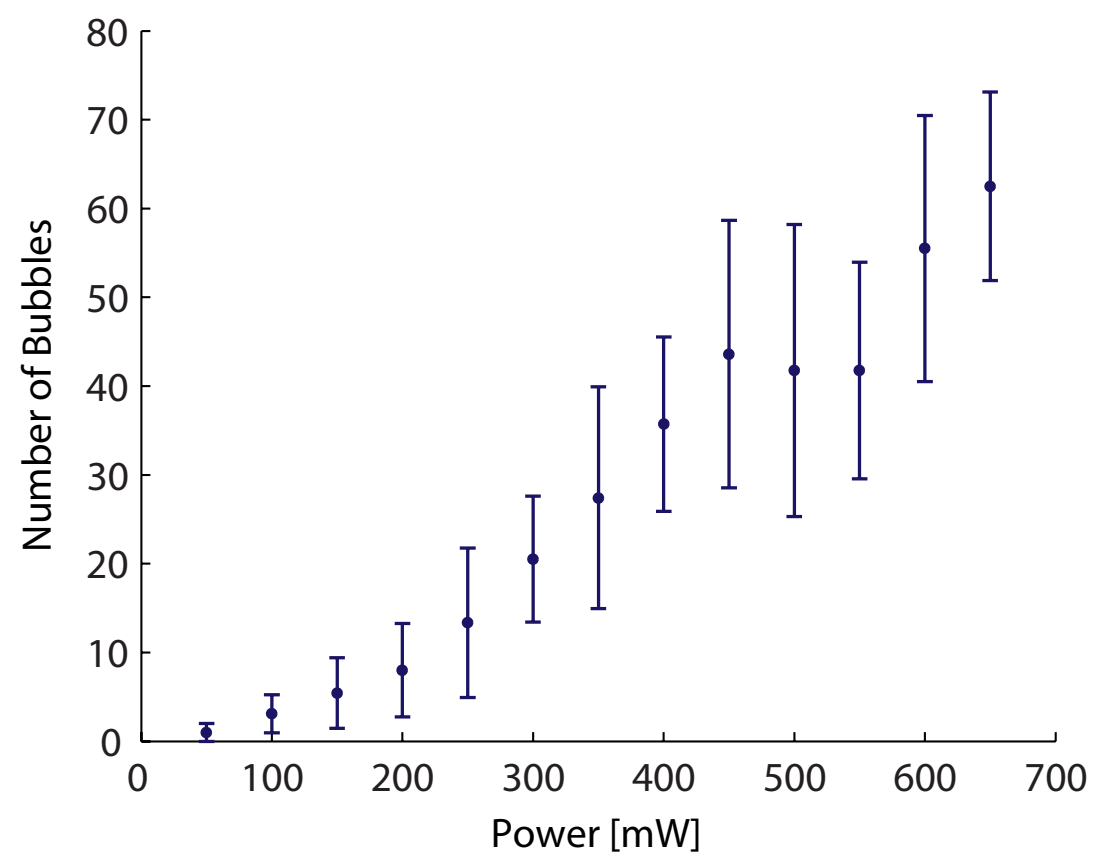

Figure 5.9: Average number of bubbles during one acoustic cycle for different driving amplitudes.

The corresponding frames in (b) obtained using flash-photography reveal the individual bubbles. From similar images the extent of the bubble cloud was measured (Figure 5.10(c)). The flattening of this graph suggests that the bubble cloud does not grow indefinitely with increasing power and remains limited to approximately 10 times the pit diameter. Similar to what was observed at $80 \mathrm{kHz}$, the pit is not deactivated by loss of gas due to pinch-off processes. It was found that the cavitation cloud can remain stable at least over an hour, only to disappear when the acoustic field became disrupted due to evaporation of the liquid.

\subsubsection{Interaction of cloud cavitation}

In the previous section it was shown that in the presence of a single micropit bubble, cavitation activity is initiated and sustained which would otherwise not occur under similar conditions. A natural solution to augment this activity is to introduce additional micropits. Indeed, as shown in the images in Figure 5.11 from a second micropit another cavitation bubble cloud is generated. Here the two micropits both of $30 \mu \mathrm{m}$ in diameter are $1000 \mu \mathrm{m}$ apart. At low 

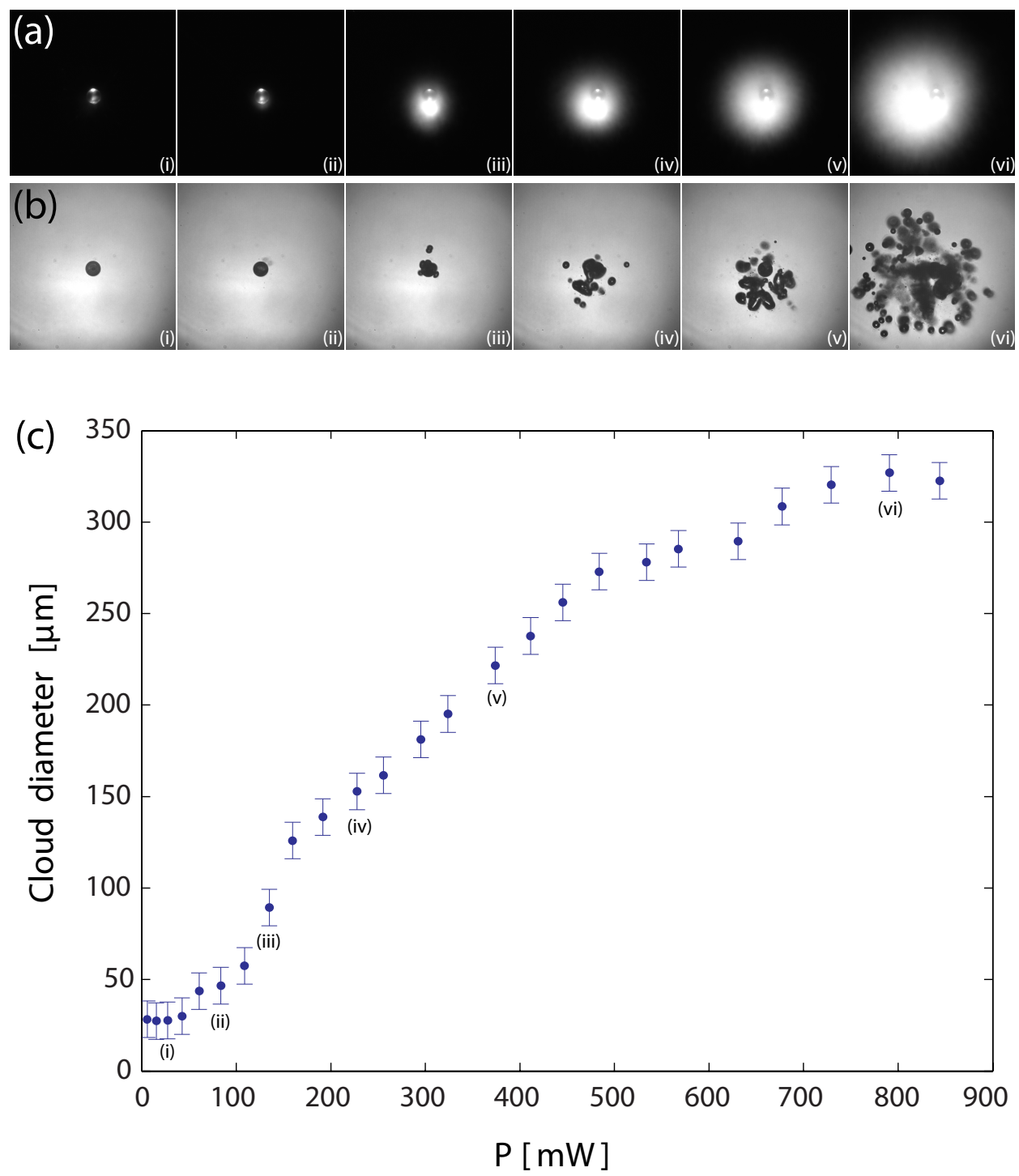

Figure 5.10: (a) Six frames from the $30 \mu \mathrm{m}$ diameter micropit bubble response for increasing driving amplitude obtained with dark field illumination. (b) The corresponding response for the single micropit bubble and its induced cavitation cloud obtained with ultrashort flash photography. (c) Graph of the diameter of the cloud for increasing driving power. The frames (i)-(vi) in (a) and (b) are indicated in the graph. 
driving amplitude the individual clouds appear similar to the single pit system described in the previous section. At elevated pressure amplitude however the center of mass of the clouds tends to deviate towards the neighboring micropit (Figure 5.11 (iv)). Above a second pressure threshold the clouds deviate to such an extent that they merge at the midpoint (Figure 5.11 (v) and (vi)). The transition between the two states occurs just above $648 \mathrm{~mW}$ driving amplitude (iii) and is quite abrupt, meaning that the intermediate states between (iv) and ( $v$ ) are either unstable or very sensitive to pressure. At higher amplitude the cloud broadens, similar to the case of a single micropit. Likewise, further increase of driving amplitude does not produce a noticeable expansion of the cavitation cloud. The joining of the clouds results in a peculiar ribbon-like structure with a wider section in the middle measuring some $200 \mu \mathrm{m}$ in diameter.

In order to illustrate the dynamics of the bubbles in the clouds a sequence of frames from a high-speed recording (Brandaris, $1.48 \mathrm{Mfps}$ ) is shown in Figure 5.12. The micropits are located in the lower-left and upper-right corners. The first frame shows the bubble cloud with the bubbles at maximum expansion (similar to the frames (v) and (vi) in Figure 5.11 (b)). During the following cycles $(5 \mu \mathrm{s}$ duration) the majority of the bubbles in the cloud collectively collapse and expand again. A few considerably larger bubbles can be observed which, due to their size, exhibit a phase difference with respect to the response of the other bubbles. For example the large bubble close to the lower-left corner continues its collapse until about $4 \mu \mathrm{s}$ ( 7 th frame) while the other bubbles have already started expansion. Similar to the single micropit bubble cloud, the bubbles display considerable coalescence, fission and deformation, in particular the larger bubbles, probably stimulated by the proximity to the substrate. It is therefore difficult to track individual bubbles in these images. However the recordings do reveal that, on average, bubbles translate after pinch-off from both micropits towards the center of the cloud. In this region a mist of small microbubbles can be observed during the high pressure phase of the acoustic cycle (e.g. at $2 \mu \mathrm{s}$ and $12.2 \mu \mathrm{s})$. These small bubbles may result from increased bubble fragmentation taking place in this region combined with dissolution. Also in this case, the remarkable stability of the cloud suggests that the process is strongly dependent on diffusive transport of dissolved gas. In addition, the recordings shown in Figures 5.10 and 5.11 demonstrate the resilience of the micropit bubbles. Evidently, even the strong pressure fluctuations associated with multiple bubble expansions and collapses are insufficient to destabilize the crevice bubble. 


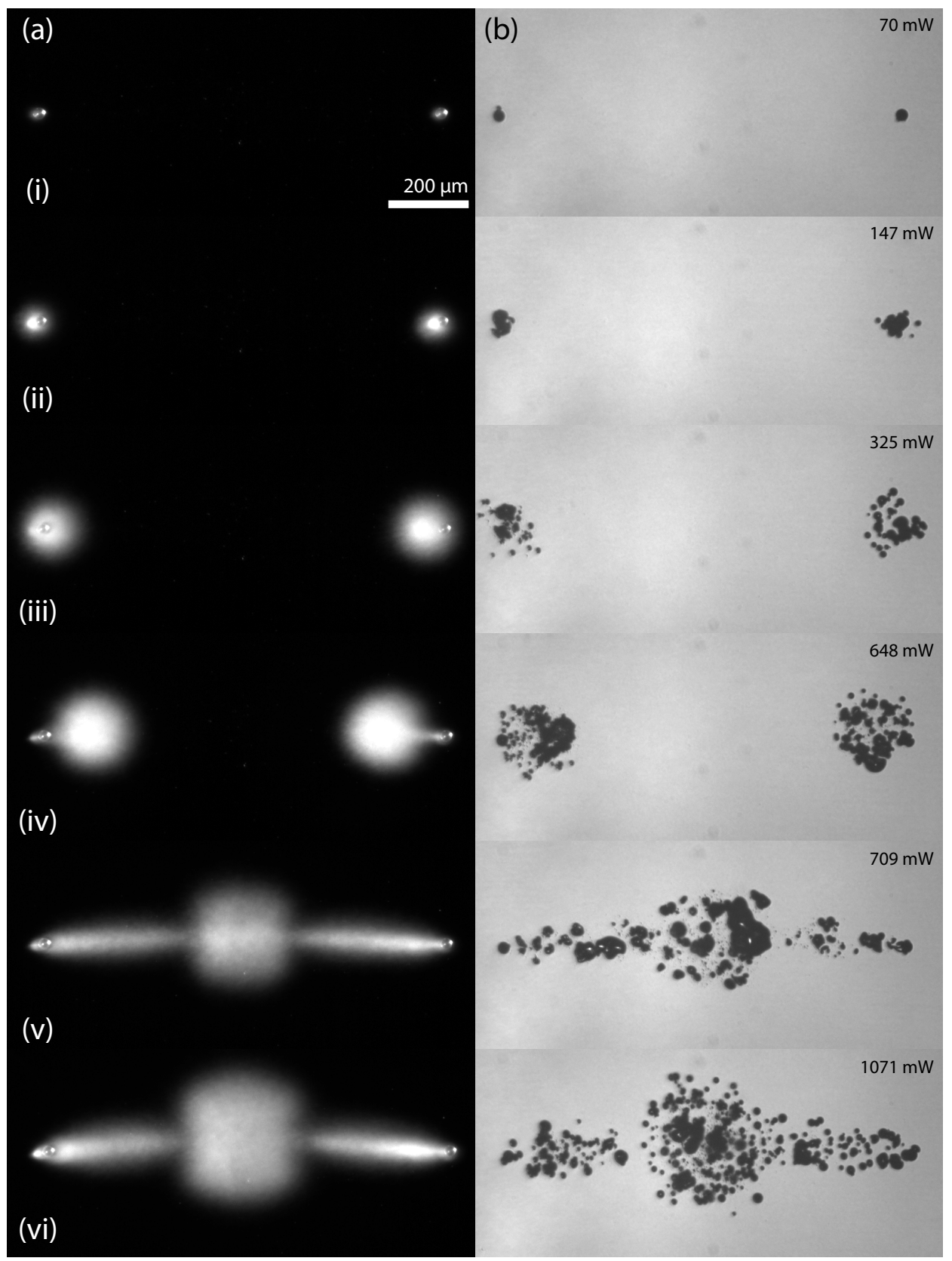

Figure 5.11: The development of the bubble cloud originating from two micropits of 30 $\mu \mathrm{m}$ diameter, $1000 \mu \mathrm{m}$ apart. The 6 rows correspond to 6 steps of increasing acoustic amplitude. The left column illustrates the time averaged extent of the cavitation cloud. In the right column the individual bubbles can be distinguished, here taken at approximately maximum expansion. 

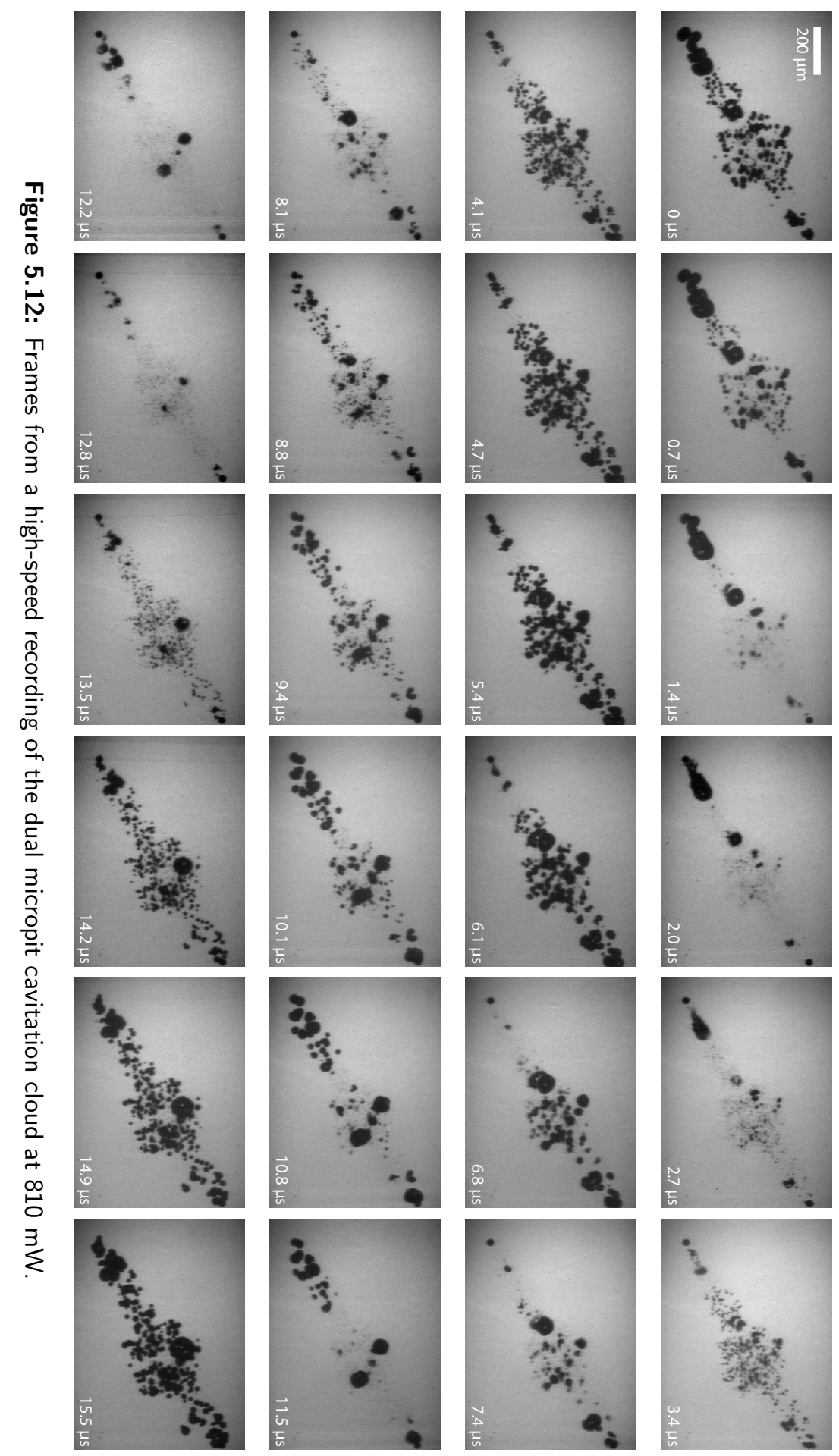

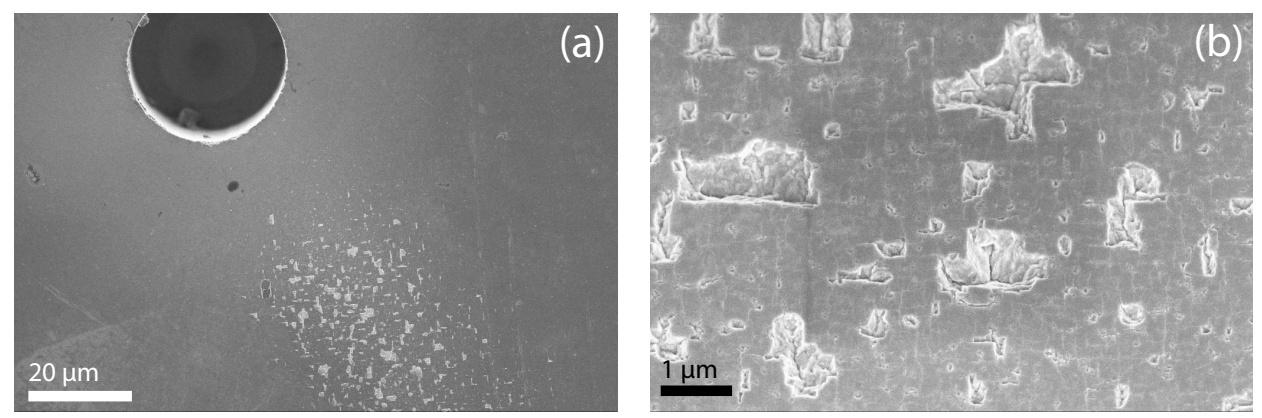

Figure 5.13: (a) Cavitation induced surface damage next to the micropit and detailed in (b).

\subsubsection{Surface damage}

An interesting finding after several hours of experimentation was a pattern of surface damage on the substrate which was consistent with the region of cavitation activity (Figure 5.13). Cavitation damage is a well known phenomenon [25, 26] and is an illustration of the enormous stresses which can be exerted by cavitation bubbles onto solid surfaces. The cumulative effect of multiple bubble collapses result in material fatigue and eventually damage. The SEM-images shown in Figure 5.13 reveal that small segments with dimensions typically less than $1 \mu \mathrm{m}$ are chipped out of the surface. Note that the shapes of the damaged sections seem to coincide with the crystal orientation planes of the crystalline structure of the silicon substrate.

Another demonstration of the forces induced by the collapse of cavitation bubbles is the occurrence of shock waves. Here, the ultrashort flash photography method enabled shadowgraphy imaging of these waves (Figure 5.14). The shock wave shown in the image originates from the collapse of the bubble cluster indicated by the red arrow. Assuming the wave to move at a velocity equal to the speed of sound in water (approximately $1500 \mathrm{~m} / \mathrm{s}$ ) the collapse occurred some $0.22 \mu \mathrm{s}$ before this image was taken. Violent bubble collapse is also associated with the generation of surface damage therefore it is possible that shock wave emission is an indication of such an event. Images similar to Figure 5.14 show circular shock wave emissions originating from many different locations within the cavitation cloud. 


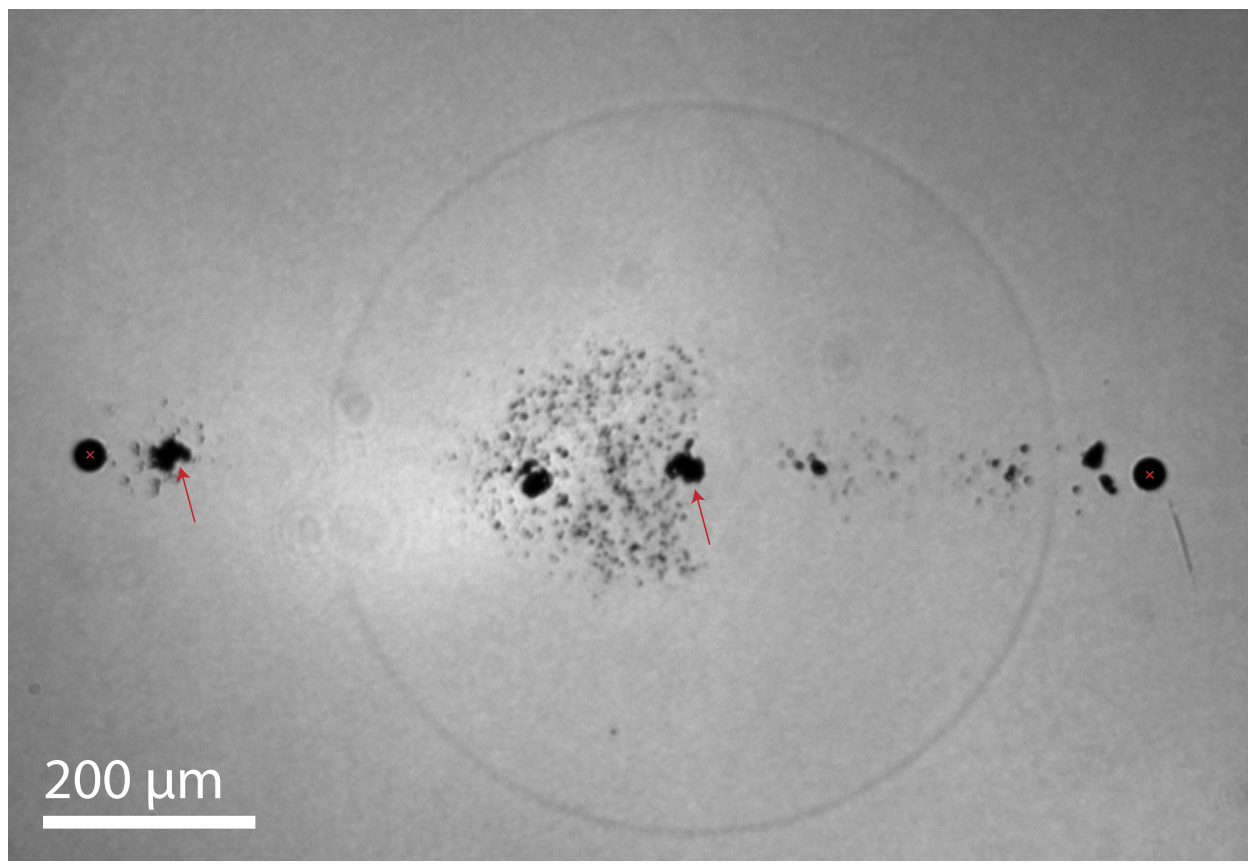

Figure 5.14: A shock wave originating from a cavitation bubble collapse close to the center of the dual-micropit bubble cloud (indicated with the red arrow on the right). The two micropits are marked with a red cross. Parts of another shock wave from an earlier event can also be distinguished, the origin of which is indicated with the left arrow.

\subsection{Discussion \& conclusion}

In this chapter we have demonstrated that the introduction of artificially created crevice bubbles into an acoustically insonified liquid can result in a myriad of acoustic cavitation related phenomena. Streams of microbubbles are released into the liquid and cavitation bubble clouds are formed at elevated acoustic pressures. The remarkable stability of these processes is enabled by the diffusion of gas back into the micropit bubble. The results of the exploratory study described in this chapter, confirms the common assumption that cavitation activity can be initiated and enhanced by the presence of crevice bubbles. However, the mechanism with which bubble release transpires is different from bubble pinch-off occurring in e.g. nucleate boiling and supersaturated liquids. In these heat and gas diffusion driven processes, the bubble displays unstable growth outside the perimeter of the pit after which it detaches due to buoyancy or drag forces. The inception of a cavitation bubble due to pinch-off from 
a micropit bubble does not appear to involve such nucleation and subsequent detachment. Here, the released bubbles are typically smaller than the micropit bubble and may result from large amplitude capillary waves which locally "fold" the liquid-gas interface into a singularities.

In this study two different frequencies were applied, both displaying distinct types of bubble generation and cavitation activity. Evidently for improved understanding a much larger parameter space should be explored. In particular the effect of driving frequency, amplitude, pit dimensions and numbers are of interest.

Clearly, the use of artificial crevice bubbles constitutes a new method to initiate and enhance cavitation at any desired location, provided the liquid and acoustic parameters are appropriate. The unique control over cavitation activity enables detailed investigation of acoustic cavitation and its effects such as shock wave emission, damage formation or, as will be demonstrated in the next chapter, sonochemistry. The introduction of micropits into a system may also proof beneficial for many applications based on acoustic cavitation. 


\section{Bibliography}

[1] K. Yasuda, T. Torii, K. Yasui, Y. lida, T. Tuziuti, M. Nakamura, and $\mathrm{Y}$. Asakura. Enhancement of sonochemical reaction of terephthalate ion by superposition of ultrasonic fields of various frequencies. Ultrason. Sonochem., 14(6):699-704, 2007.

[2] S. Muthukumaran, K. Yang, A. Seuren, S. Kentish, M. Ashokkumar, G. W. Stevens, and F. Grieser. The use of ultrasonic cleaning for ultrafiltration membranes in the dairy industry. Sep. Purif. Technol., 39(1-2):99 - 107, 2004.

[3] T. J. Mason, L. Paniwnyk, and J. P. Lorimer. The uses of ultrasound in food technology. Ultrason. Sonochem., 3(3):S253 - S260, 1996.

[4] R. E. Apfel. Acoustic cavitation inception. Ultrasonics, 22(4):167 - 173, 1984.

[5] S. Helbig, S. Urban, E. Klein, and S. Singh. Impact of megasonic process conditions on pre and sub-resolution assist feature damage. Photomask Technology, 7122(1):712210, 2008.

[6] W. J. Greenleaf, M. E. Bolander, G. Sarkar, M. B. Goldring, and J. F. Greenleaf. Artificial cavitation nuclei significantly enhance acoustically induced cell transfection. Ultrasound Med. Biol., 24(4):587 - 595, 1998.

[7] D. M. Hallow, A. D. Mahajan, T. E. McCutchen, and M. R. Prausnitz. Measurement and correlation of acoustic cavitation with cellular bioeffects. Ultrasound Med. Biol., 32(7):1111 - 1122, 2006.

[8] Kukizaki $\mathrm{M}$ and $\mathrm{M}$. Goto. Size control of nanobubbles generated from shirasu-porous-glass (spg) membranes. J. Membr. Sci., 281(1-2):386 396, 2006.

[9] B. Dollet, W. van Hoeve, J. P. Raven, P. Marmottant, and M. Versluis. Role of the channel geometry on the bubble pinch-off in flow-focusing devices. Phys. Rev. Lett., 100(3):034504, 2008.

[10] E. N. Harvey, D. K. Barnes, W. D. McElroy, A. H. Whiteley, D. C. Pease, and K. W. Cooper. Bubble formation in animals. i. physical factors. J Cell Comp Physiol., 24(1):1-22, 1944.

[11] A. A. Atchley and A. Prosperetti. The crevice model of bubble nucleation. J. Acoust. Soc. Am., 86(3):1065-1084, 1989. 
[12] N. Bremond, M. Arora, C. D. Ohl, and D. Lohse. Cavitation on surfaces. Journal of Physics: Condensed Matter, 17(45):S3603, 2005.

[13] N. Bremond, M. Arora, C. D. Ohl, and D. Lohse. Controlled multibubble surface cavitation. Phys. Rev. Lett., 96(22):224501, 2006.

[14] B. M. Borkent, S. Gekle, A. Prosperetti, and D. Lohse. Nucleation threshold and deactivation mechanisms of nanoscopic cavitation nuclei. Physics of Fluids, 21(10):102003, 2009.

[15] S. F. Jones, G. M. Evans, and K. P. Galvin. Bubble nucleation from gas cavities - a review. J. Coll. Interf. Sci., 80(1):27 - 50, 1999.

[16] G. Liger-Belair, Mi. Vignes-Adler, C. Voisin, B. Robillard, and P. Jeandet. Kinetics of gas discharging in a glass of champagne: The role of nucleation sites. Langmuir, 18(4):1294-1301, 2002.

[17] C. Y. Han and P. Griffith. The mechanism of heat transfer in nucleate pool boiling. Technical report, Massachusetts Institute of Technology. Heat Transfer Laboratory, 1962.

[18] C. H. Wang and V. K. Dhir. Effect of surface wettability on active nucleation site density during pool boiling of water on a vertical surface. Journal of heat transfer, 115(3):659-669, 1993.

[19] D. E. Hughes and W. L. Nyborg. Cell disruption by ultrasound. Science, 138(3537):pp. 108-114, 1962.

[20] C. T. Chin, C. Lancée, J. Borsboom, F. Mastik, M. E. Frijlink, N. de Jong, M. Versluis, and D. Lohse. Brandaris 128: A digital 25 million frames per second camera with 128 highly sensitive frames. Rev. Sci. Instr., 74(12): 5026-5034, 2003.

[21] F. P. Schäfer, W. Schmidt, and J. Volze. Organic dye solution laser. Applied Physics Letters, 9(8):306-309, 1966.

[22] G. D. Crapper. An exact solution for progressive capillary waves of arbitrary amplitude. J. Fluid Mech., 2(06):532-540, 1957.

[23] A. Eller and H. G. Flynn. Rectified diffusion during nonlinear pulsations of cavitation bubbles. J. Acoust. Soc. Am., 37(3):493-503, 1965.

[24] D. L. Miller. Particle gathering and microstreaming near ultrasonically activated gas-filled micropores. J. Acoust. Soc. Am., 84(4):1378-1387, 1988. 
[25] D. Krefting, R. Mettin, and W. Lauterborn. High-speed observation of acoustic cavitation erosion in multibubble systems. Ultrason. Sonochem., 11(3-4):119 - 123, 2004.

[26] M. Dular and A. Osterman. Pit clustering in cavitation erosion. Wear, 265 (5-6):811 - 820, 2008. 


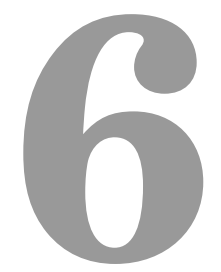

\section{Micropit bubble enhanced sonochemistry ${ }^{1}$}

In principle, sonochemical reactors are attractive options for many applications in water treatment, the synthesis of fine chemicals, pharmaceutics and others. Widespread adoption of these reactors is hampered however by their low efficiency due, in its turn, to the difficulty of controlling the bubble activity responsible for sonochemical effects. This chapter presents a new approach to the design of sonochemical reactors which results in a greatly increased efficiency and controllability. The novel reactor consists of a vessel the bottom of which is a silicon surface on which a number of pits are micromachined. The pits entrap gas which, upon ultrasonic excitation, is emitted in the form of a stream of microbubbles. The gas content of the pits is not depleted but is replenished by diffusion and the emission of microbubbles can continue for hours. Visual and chemical evidence is presented showing that the microbubbles are chemically active at ultrasonic amplitudes well below those necessary for sonochemical activity in conventional reactors.

\subsection{Introduction}

The phenomenon of cavitation, that is, the growth and implosion of gas/vapor bubbles in a liquid, is a process which can locally generate extreme temperatures of several thousand Kelvin [1] and, for this reason, is exploited in sonochemistry to enhance chemical conversion. This feature opens the perspective of high-temperature, high-pressure large scale systems and therefore holds the promise of constituting a "green chemistry" with a multitude of possible ap-

\footnotetext{
${ }^{1}$ Published as: David Fernandez Rivas, Andrea Prosperetti, Aaldert G. Zijlstra, Detlef Lohse and Han J. G. E. Gardeniers, Efficient Sonochemistry through Microbubbles Generated with Micromachined Surfaces, Angewandte Chemie 122, 50 (2010), 9893-9895
} 
plications in water treatment [2], material synthesis, the food industry [3] and others. The "ideal" sonochemical reactor from a theoretical point of view is a single bubble trapped in an acoustically driven flask, such as in single bubble sonoluminescence [4]. There the bubble, in which argon accumulates [5], collapses periodically and in a reproducible way, as the collapse does not desintegrate the bubble. Reactants are sucked into the bubble at expansion and reaction products leave the bubble at collapse; typical temperatures achieved are $15000 \mathrm{~K}$ [6, 7]. Another advantage is that thanks to these ideal conditions the problem is accessible to a thorough theoretical treatment whose results are in good agreement with the experimental findings [4, 6]. The downside is that the absolute chemical yields are only tiny, as the ambient size of such bubbles is in the micrometer regime. For applications, typical sonochemical reactors, such as ultrasonic baths or vessels with ultrasound horns attached to their walls, are considerably larger than the active region in which cavitation occurs, which is defined by the ultrasound field that the transducer generates in the reactor. The difficulty of matching the acoustic cavitation activity with the reactor dimensions so that the complete volume of reactant can be stimulated adds to the low efficiency of most sonochemical processes. Attempts aimed at configuration optimization have mostly had limited success [8-11].

In this chapter a new approach is presented to address this problem based on the premise that a significant gain in efficiency may be obtained if the location of the nucleation of bubbles, which subsequently will cavitate due to ultrasound, can be accurately controlled. Achieving this objective will give us control over the spatial distribution of cavitation events, and therewith also over the actual volume of liquid that can be exposed to sonochemical effects.

The nucleation and formation of bubbles due to ultrasound is mostly heterogeneous, that is, it relies on pockets of gas trapped and stabilized inside randomly existing crevices in container walls or particles suspended in the bulk fluid rendering it extremely difficult to control and predict. The theory developed for bubble nucleation from crevices [12, 13] leads the way to control nucleation. As shown in recent work [13-16] and in the previous chapter, stable and monodisperse cavitation nuclei can be formed by trapping gas in pits micromachined in a silicon surface. We use such pits which serve as artificial crevices for the inception of cavitation to achieve higher sonochemical yields at ultrasound powers which would otherwise not produce a significant chemical effect. 


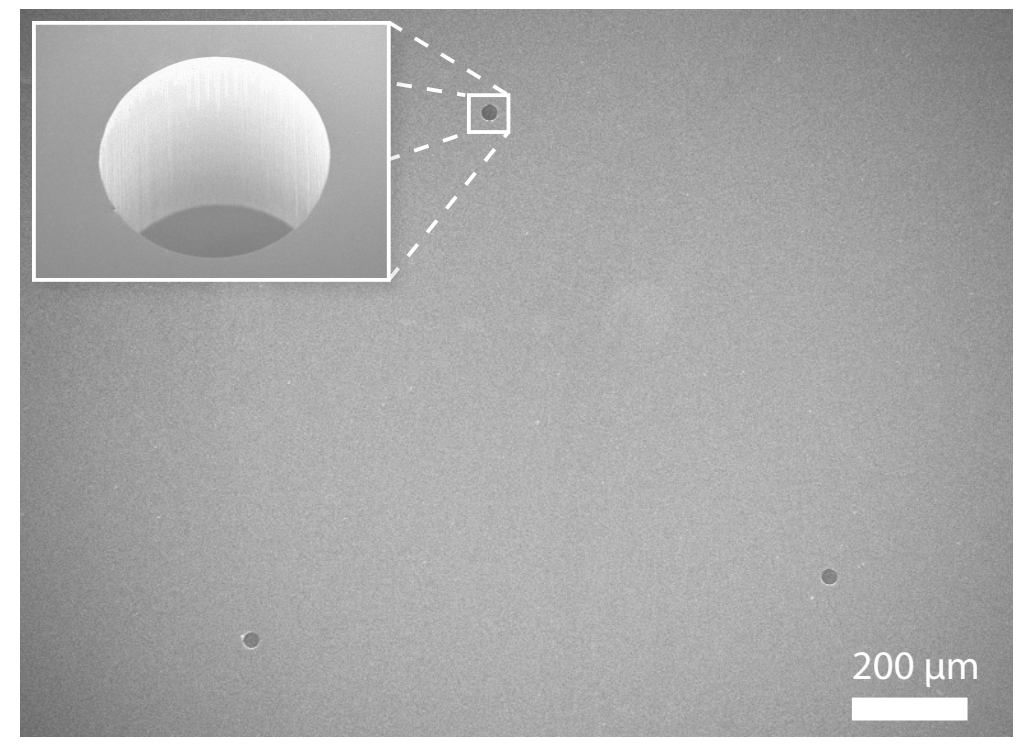

Figure 6.1: Pits etched in a silicon substrate. Top view of the 3-pit set with $30 \mu \mathrm{m}$ diameter micropits.

\subsection{Experimental methods and materials}

\subsubsection{Silicon micromachining:}

Three different configurations of pits were used. The pits had the same diameter $(30 \mu \mathrm{m})$ and were arranged in sets of one, two (in a line, see previous chapter) or three (in a triangle, see Figure 6.1) at a distance of $1000 \mu \mathrm{m}$ from each other. The substrates were micromachined similarly to the process described in section 5.2.1. The machined diced silicon square pieces of $1 \mathrm{~cm}$-side were placed on the bottom of a small glass container which contained a liquid volume of $300 \mu \ell$.

\subsubsection{The acoustic field}

The reaction chamber was a glass container of $2.5 \mathrm{~cm}$ outer diameter, $1.5 \mathrm{~cm}$ inner diameter and depth of $3 \mathrm{~mm}$, and bottom thickness of $2 \mathrm{~mm}$. the bottom thickness matched the quarter-wavelength vibration imparted by a piezo Ferroperm PZ27 $6 \mathrm{~mm}$ thick with diameter of $2.5 \mathrm{~cm}$, glued to the bottom of the reaction chamber. The top of the glass container was capped with a rubber ring and a glass slide to avoid evaporation of the sample during the experiments (see Figure 6.2). 


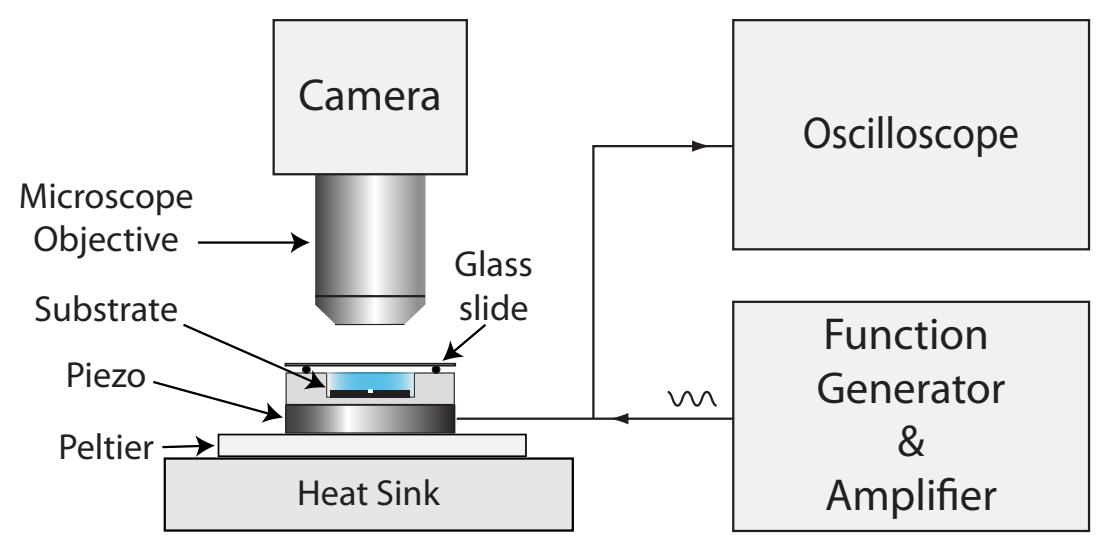

Figure 6.2: Experimental setup

A sinusoidal standing acoustic field of $200 \mathrm{kHz}$ was generated by a Hewlett Packard model 33120A arbitrary waveform generator, amplified with a SONY TA-FB740R QS amplifier. The voltage, current and phase difference provided to the piezoelectric element were measured with a Tektronix DPO 4034 oscilloscope and Tektronix current probe CTA-2.

Three different power settings were used: low $(74 \mathrm{~mW})$, medium $(182 \mathrm{~mW})$ and high $(629 \mathrm{~mW})$. During the experiments, the bottom surface of the piezoelectric element was in contact with a Peltier element (Marlow Industrial) to keep the temperature at a constant value of approximately $25^{\circ} \mathrm{C}$.

\subsubsection{Luminol visualization and determination of the radical for- mation rate}

The homolytic cleavage of $\mathrm{H}_{2} \mathrm{O}$ by sonolysis was investigated using Luminol and terephthalic acid. Luminol is a well-established indicator for the visualization of active sonochemical regions, because it reacts with $\mathrm{OH}$ radicals, whereupon reaction gives luminescence with an intensity proportional to the amount of radicals produced [17]. A solution of $1 \times 10^{-3} \mathrm{~mol} / \ell$ Luminol and $1 \times 10^{-4} \mathrm{~mol} / \ell$ hydrogen peroxide was prepared with adjusted $\mathrm{pH}=12$ by adding $\mathrm{Na}_{2} \mathrm{CO}_{3}$, as described in [18].

Conversion of terephthalic acid to 2-hydroxyterephthalic acid (HTA) was taken as a quantitative measure for the concentration of $\mathrm{OH}$ radicals formed by the ultrasound induced bubble activity. A calibration graph for the fluorescence intensity as a function of HTA concentration was obtained following steps similar to those described in [19]. Fluorescence was measured using a spectrofluorometer (TECAN Sapphire) with an excitation wavelength of $310 \mathrm{~nm}$ and 
an emission wavelength of $429 \mathrm{~nm}$. A graph of fluorescence intensity against HTA concentration was plotted and gave a straight line of positive slope for concentrations. The Terephthalic acid solution to be used as dosimeter was prepared by mixing $0.332 \mathrm{~g}$ of Terephthalic acid (Sigma-Aldrich, $2.0 \times 10^{-3}$ $\mathrm{mol} / \ell), 0.200 \mathrm{~g}$ of $\mathrm{NaOH}\left(5.0 \times 10^{-3} \mathrm{~mol} / \ell\right)$, and phosphate buffer $(\mathrm{pH} 7.4)$, prepared from $0.589 \mathrm{~g}$ of $\mathrm{KH}_{2} \mathrm{PO}_{4}\left(4.4 \times 10^{-3} \mathrm{~mol} / \ell\right)$ and $0.981 \mathrm{~g}$ of $\mathrm{Na}_{2} \mathrm{HPO}_{4}$ $\left(7.0 \times 10^{-3} \mathrm{~mol} / \ell\right.$, all from Riedel - de Häen $)$. The resulting solution was then made up to $1 \ell$ with water [20]. For each experiment run, the same amount of terephthalic acid solution $300 \mu \ell$ measured with Eppendorf micropipettes was used.

At the end of the experimental run, the solution was pipetted out of the reaction chamber and stored in the dark in sterilized vials (manufactured by $B r a n d)$ for ultrapure chemical analysis for further spectroscopic analysis. Later, $200 \mu \ell$ taken from the samples were pipetted into the wells of an assay plate (Corning Inc.) to be analyzed with the spectrofluorometer described above. The conditions for sample analysis were: gain 40, height from the well, 9000 $\mu \mathrm{m}$, integration time $100 \mu \mathrm{s}, 10$ reads per well. Excitation was at $310 \mathrm{~nm}$ and the emission scan was from 350 to $600 \mathrm{~nm}$. Each experiment was repeated at least 6 times showing acceptable consistency.

Calculation of the radical formation rate was done according to:

$$
\frac{d N_{\text {rad }}}{d t}=\frac{\left(c_{\text {end }}-c_{\text {initial }}\right) N_{A} V}{\Delta t}
$$

Here $c_{\text {end }}$ and $c_{\text {initial }}$ are the final and initial concentration in moles per volume, $N_{A}=6.02 \times 10^{23} \mathrm{~mol}^{-1}$ is Avogadro's number and $V$ is the liquid volume exposed to the ultrasound $(300 \mu \ell)$. With $c_{\text {initial }}=0$ this relation provides an estimate of the rate of radical formation for all pits and power combinations. 

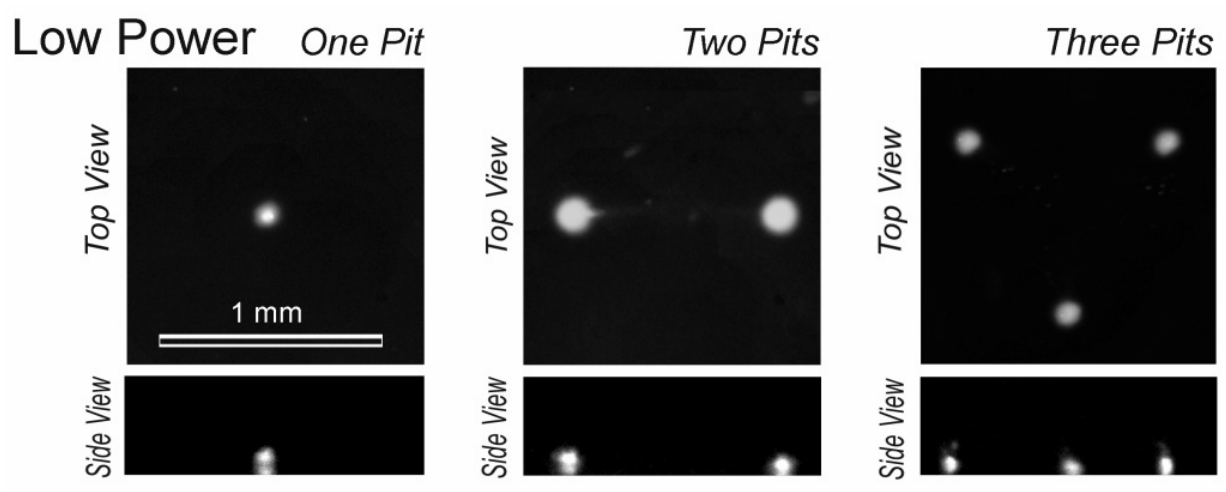

\section{Medium Power}
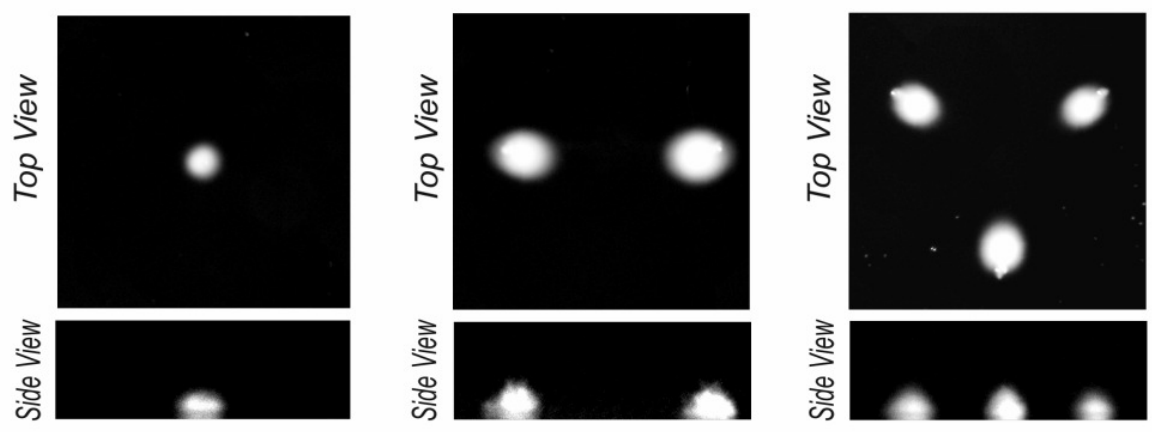

\section{High Power}
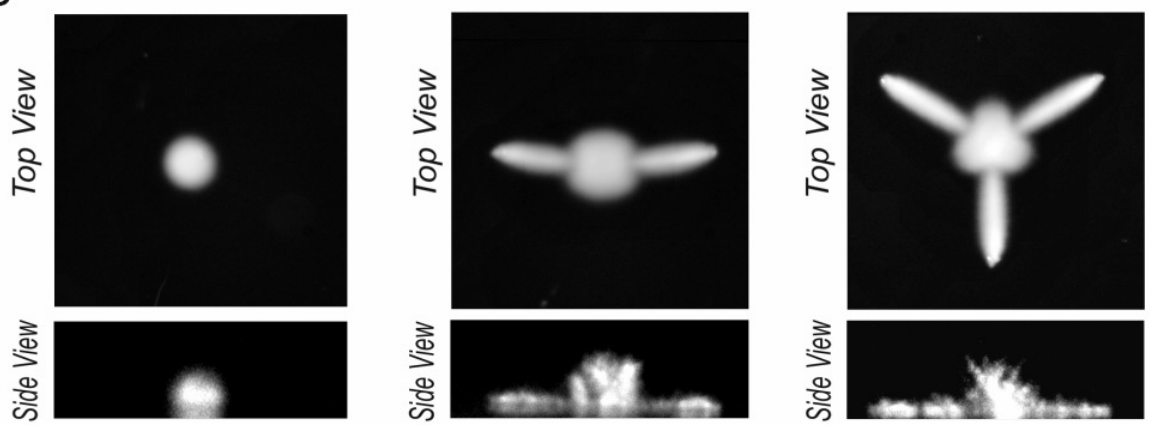

Figure 6.3: Images showing both the top and side view of the bubble structures generated from the micropits for different configurations (1,2 and 3 pits) and for increasing power level. Low power corresponds to $74 \mathrm{~mW}$, medium power to $182 \mathrm{~mW}$, and high power to $629 \mathrm{~mW}$. 


\subsection{Results}

Similar to described in the previous chapter, the micropit bubble ejects streams of microbubbles, which resemble previously reported streamers [21, 22]. The observed trajectories in Figure 6.3 are the result of the complex interplay of primary and secondary Bjerknes forces and microstreaming. For a single pit, at low and medium ultrasound powers, the microbubbles are ejected and oscillate several cycles until they dissolve or are recaptured by the micropit bubble. For low power the situation is similar for the configurations with two and three pits. With increasing power a drastic transition in the bubble flow pattern is observed. Beyond the transition the microbubbles move away from the symmetry axis of their respective pits and towards one another (see intermediate row in Figure 6.3). The microbubbles then form a dense bubble cloud traveling towards the midpoint between the two-pit or to the midpoint of the three-pit arrangement. For the three-pit configuration the microstreamers point to the center of the triangular array and form a triangular cloud of bubbles, as seen in top view.

These experiments were repeated with the water solution of luminol. In Figure 6.4 the resulting images can be seen. Imaging of the low intensity of the emitted luminescence required the use of long camera exposure times (typically $30 \mathrm{sec}-$ onds). A detailed comparison of the features in Figure 6.4 with those in Figure 6.3 shows a perfect match. Clearly, the regions of cavitation activity correspond to the regions where light is emitted due to sonochemiluminescence [17].

For a quantitative measure of radical formation, the terephthalic acid dosimetry method was used. The fluorescence intensity emission of HTA at $429 \mathrm{~nm}$ (measured ex-situ) allows us to calculate the amount of radicals generated by ultrasound induced microbubble cavitation. This measurement was performed for the three pit configurations, each at low, medium and high power and for 15 and 30 minutes process durations. In addition, experiments with the same conditions were done with a silicon chip without micropits. These measurements showed no significant evidence of radical formation. The radical generation rates (equation 6.1) resulting from off-line fluorescence measurements are shown in Figure 6.5.

The data show that the presence of pits gives a significant enhancement of the radical formation rate. The rate is stable over time and is an increasing power of the ultrasound field as expected. Radical formation increases with the number of pits, but the slope of the lines decreases as more pits are added, presumably due to the onset of interactions with the bubble clouds generated by the other pits. At high power, the bubble pattern changes dramatically (Figures 6.3 and 6.4) and is expected to lead to a different radical generation 
Low Power One Pit

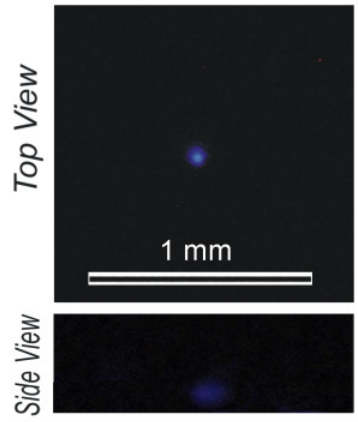

\section{Medium Power}

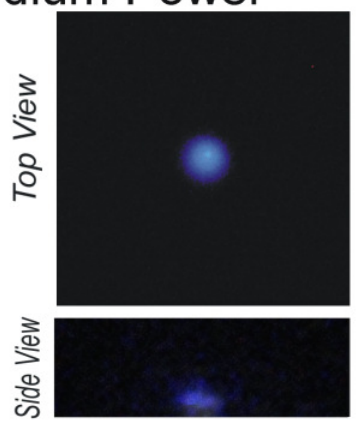

\section{High Power}

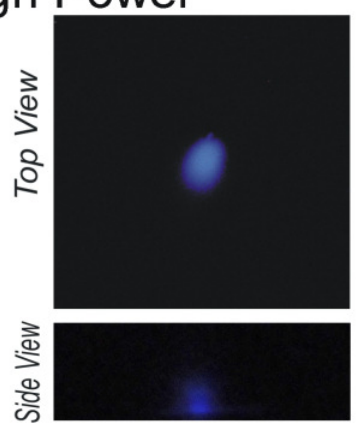

Two Pits
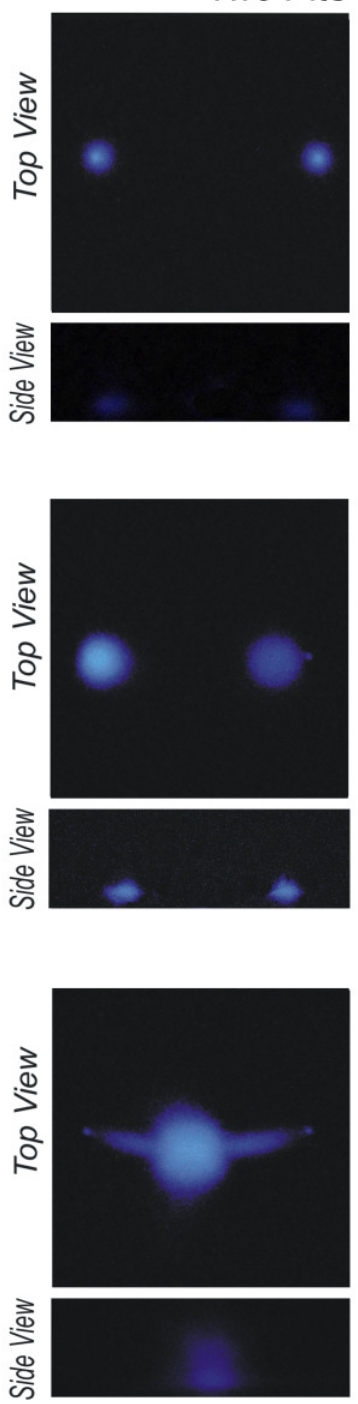

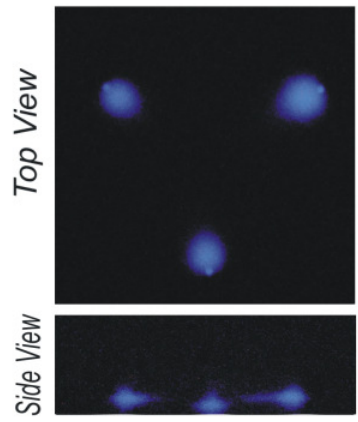

Three Pits
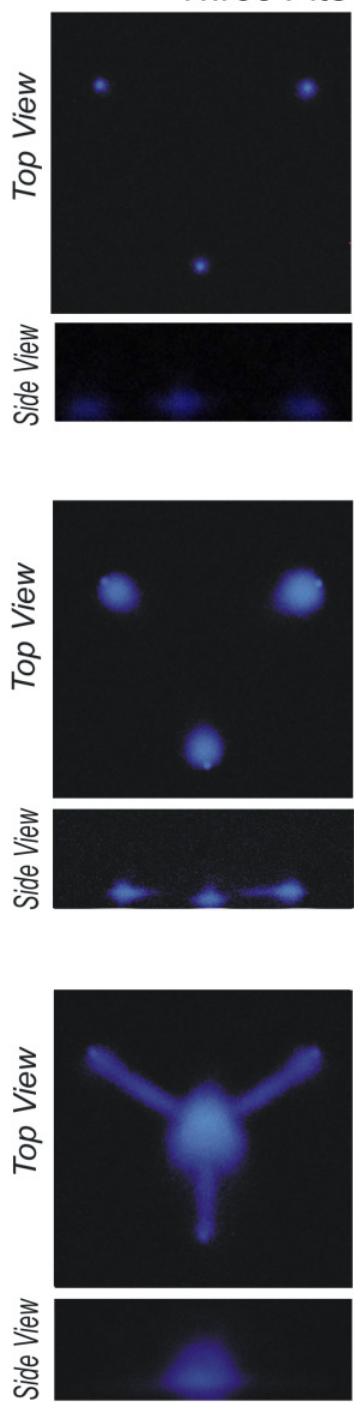

Figure 6.4: Luminol luminescence in dark room conditions. Images showing both the top and side view of the bubble structures generated from the micropits for different configurations (1,2 and 3 pits) and for increasing power level. Compare with Figure 6.3 


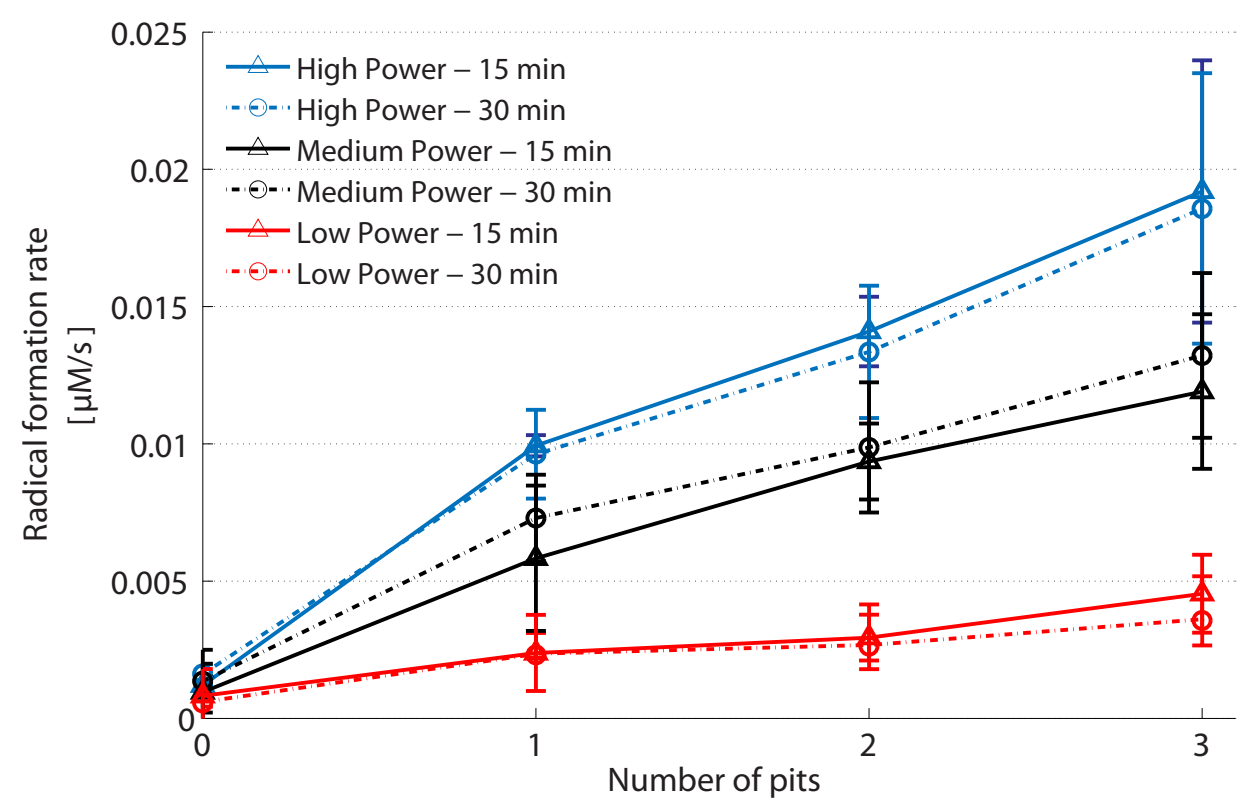

Figure 6.5: Radical formation rate vs. pits. Low power $=74 \mathrm{~mW}$, medium power $=182$ $\mathrm{mW}$ and high power $=629 \mathrm{~mW}$. The bars show the range of the experimental data

distribution over the reactor volume. The quantitative interpretation of these data is complex. Generally speaking, smaller bubbles are stiffer due to a surface tension contribution and may not grow as large during expansion, with a consequent weaker compression and lower maximum temperatures. Large bubbles, on the other hand, may not collapse spherically, especially when close to a solid surface, which would also limit their maximum compression. It is not clear, however, to what extent these general trends are relevant in the experimental situation of present concern.

The most important parameter for the evaluation of our results and comparison with the work of others is the energy efficiency defined as:

$$
X_{U S}=\frac{\Delta H}{P_{U S}} \frac{d N_{r a d}}{d t}
$$

where $\frac{d N_{\text {rad }}}{d t}$ is the radical formation rate in moles per second, $\Delta H$ is the energy required for the formation of $\mathrm{OH}$ radicals, which is equal to the enthalpy of formation associated with the following chemical reaction:

$$
\mathrm{H}_{2} \mathrm{O} \leftrightarrow \mathrm{OH}+\mathrm{H}
$$

This enthalpy has a value of $5.1 \mathrm{eV}$ per molecule [23]. $P_{U S}$ is the electric power absorbed by the transducer which can be obtained from the measured voltage, 
Table 6.1: Efficiency $\times 10^{6}$ as defined in equation 6.2

\begin{tabular}{cllll}
\hline $\begin{array}{c}\text { High power } \\
629 \mathrm{~mW}\end{array}$ & 3 pits & 2 pits & 1 pit & 0 pit \\
\hline $15 \mathrm{~min}$ & 4.5 & 3.3 & 2.3 & 0.3 \\
$30 \mathrm{~min}$ & 4.4 & 3.1 & 2.3 & 0.4 \\
\hline $\begin{array}{c}\text { Medium power } \\
182 \mathrm{~mW}\end{array}$ & 3 pits & 2 pits & 1 pit & 0 pit \\
\hline $15 \mathrm{~min}$ & 9.7 & 7.6 & 4.7 & 0.8 \\
$30 \mathrm{~min}$ & 11 & 8.0 & 5.9 & 0.1 \\
\hline Low power & & & & \\
$74 \mathrm{~mW}$ & 3 pits & 2 pits & 1 pit & 0 pit \\
\hline $15 \mathrm{~min}$ & 9.1 & 5.9 & 4.8 & 1.6 \\
$30 \mathrm{~min}$ & 7.1 & 5.2 & 4.6 & 1.1 \\
\hline
\end{tabular}

current and their phase difference. The efficiency as defined in Equation 6.2 is shown in Table 6.1 for each of the configurations studied.

\subsection{Discussion and conclusions}

From the values in Table 6.1 we can draw the following conclusions: (i) Comparing the efficiency values of the chips with one or several pits with those of the chip with no pits, there is an efficiency increase by an order of magnitude, which demonstrates that the pits on the reactor wall give a considerable enhancement. (ii) The efficiency obtained even with such a low number of pits is close to the highest efficiencies reported in the literature with conventional sonochemical reactors. [20, 24--30] A detailed comparison is hardly possible due to the use of different frequencies, chemical dosimeters and other physical parameters.

From the values of Table 6.1 it can be seen that the most efficient setting is medium power in all pit configurations. Several factors may explain this observation. At high power, the microbubble collapse is more catastrophic which results in a smaller compression and heating of the gas. In the case of two- and three-pit configuration, the microbubbles stay longer in the most active zone close to the substrate while traveling parallel to the surface; but the collapse of microbubbles close to the surface is highly non spherical, again reducing the maximum compression and heating of the gas. The microbubbles in the dense cloud at the midpoint may also have a weaker collapse due to the 

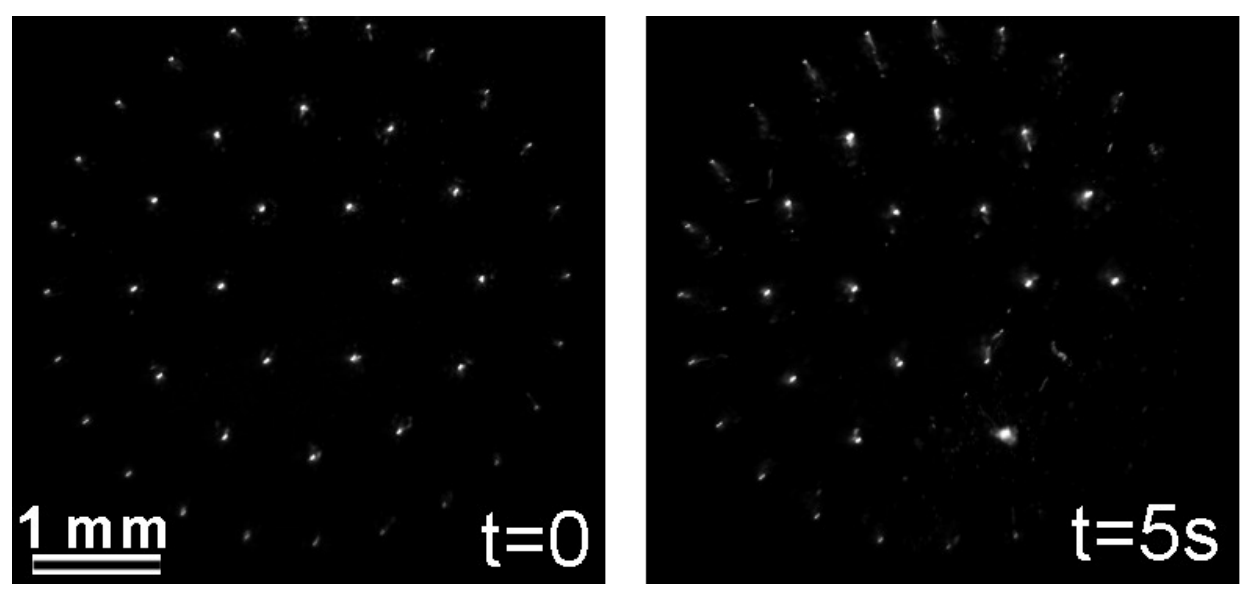

Figure 6.6: Microscope image under normal light conditions showing an arrangement with a higher number of pits (42). Here similar ejections of microbubbles from the micropit bubbles occur as described in this and previous chapter.

shielding by the outer microbubbles.

It has to be stressed that our results were obtained with only a very small active region, with state-of-the-art microfabrication procedures it is straightforward to increase the number of pits (see previous chapter and Figure 6.6). Based on our results in Table 6.1 and Figure 6.5. increasing the number of pits should give an increase in energy efficiency by a factor of 100 or more. This estimate is based on a linear extrapolation of the one-, two- and three-pit configuration at medium power settings.

Our results indicate that the introduction of micromachined pits on the surface of a reactor wall attached to an ultrasound transducer can boost sonochemical efficiency. The underlying mechanism is the production of microbubble cavitation clouds from the stable gas pockets that form in these pits. 


\section{Bibliography}

[1] K. S. Suslick and D. J. Flannigan. Inside a collapsing bubble: Sonoluminescence and the conditions during cavitation. Annu. Rev. Phys. Chem., 59:659-683, 2008.

[2] P. R. Gogate. Application of cavitational reactors for water disinfection: Current status and path forward. J. Environ. Manage., 85:801-815, 2007.

[3] M. Ashokkumar, D. Sunartio, S. Kentish, R. Mawson, L. Simons, K. Vilkhu, and C. Versteeg. Modification of food ingredients by ultrasound to improve functionality: A preliminary study on a model system. Innov. Food Sci. Emerg, Technol., 9(2):155-160, 2008.

[4] M. P. Brenner, S. Hilgenfeldt, and D. Lohse. Single-bubble sonoluminescence. Rev. Mod. Phys., 74(2):425-484, 2002.

[5] D. Lohse, M. P. Brenner, T. F. Dupont, S. Hilgenfeldt, and B. Johnston. Sonoluminescing air bubbles rectify argon. Phys. Rev. Lett., 78(7):13591362, 1997.

[6] R. Toegel and D. Lohse. Phase diagrams for sonoluminescing bubbles: A comparison between experiment and theory. J. Chem. Phys., 118:1863, 2003.

[7] D. J. Flannigan and K. S. Suslick. Plasma formation and temperature measurement during single-bubble cavitation. Nature, 434(7029):52-55, 2005.

[8] K. Yasuda, T. Torii, K. Yasui, Y. lida, T. Tuziuti, M. Nakamura, and $\mathrm{Y}$. Asakura. Enhancement of sonochemical reaction of terephthalate ion by superposition of ultrasonic fields of various frequencies. Ultrason. Sonochem., 14(6):699-704, 2007.

[9] Z. Xu, C. Y. Ma, J. Y. Xu, and X. J. Liu. Dynamical properties of iodine release in potassium iodide solution under combination of ultrasound and light irradiations. Ultrason. Sonochem., 16(4):475-480, 2009.

[10] S. Koda, K. Tanaka, H. Sakamoto, T. Matsuoka, and H. Nomura. Sonochemical efficiency during single-bubble cavitation in water. J. Phys. Chem. A, 108(52):11609-11612, 2004.

[11] T. Tuziuti, K. Yasui, T. Kozuka, A. Towata, and Y. lida. Enhancement of sonochemical reaction rate by addition of micrometer-sized air bubbles. $J$. Phys. Chem. A, 110(37):10720-10724, 2006. 
[12] A. A. Atchley and A. Prosperetti. The crevice model of bubble nucleation. J. Acoust. Soc. Am., 86(3):1065-1084, 1989.

[13] B. M. Borkent, S. Gekle, A. Prosperetti, and D. Lohse. Nucleation threshold and deactivation mechanisms of nanoscopic cavitation nuclei. Physics of Fluids, 21(10), 2009.

[14] N. Bremond, M. Arora, C. D. Ohl, and D. Lohse. Controlled multi-bubble surface cavitation. Phys. Rev. Lett., 96:224501, 2006.

[15] N. Bremond, M. Arora, S. M. Dammer, and D. Lohse. Interaction of cavitation bubbles on a wall. Physics of Fluids, 18(12), 2006.

[16] P. Marmottant, J. P. Raven, H. Gardeniers, J. G. Bomer, and S. Hilgenfeldt. Microfluidics with ultrasound-driven bubbles. J. Fluid Mech., 568: 109-118, 2006.

[17] A. Henglein, R. Ulrich, and J. Lilie. Luminescence and chemical action by pulsed ultrasound. J. Am. Chem. Soc., 111(6):1974-1979, 1989.

[18] B. Felver, D. C. King, S. C. Lea, G. J. Price, and A. D. Walmsey. Cavitation occurrence around ultrasonic dental scalers. Ultrason. Sonochem., 16(5): 692-697, 2009.

[19] T. J. Mason, J. P. Lorimer, D. M. Bates, and Y. Zhao. Dosimetry in sonochemistry - the use of aqueous terephthalate ion as a fluorescence monitor. Ultrason. Sonochem., 1(2):S91-S95, 1994.

[20] Y. lida, K. Yasui, T. Tuziuti, and M. Sivakumar. Sonochemistry and its dosimetry. Microchem. J., 80(2):159-164, 2005.

[21] T. G. Leighton. Bubble population phenomena in acoustic cavitation. Ultrason. Sonochem., 2(2):S123-S136, 1995.

[22] E. A. Neppiras. Acoustic cavitation. Phys. Rep., 61(3):159-251, 1980.

[23] R. Toegel, S. Hilgenfeldt, and D. Lohse. Suppressing dissociation in sonoluminescing bubbles: The effect of excluded volume. Phys. Rev. Lett., 88 (3), 2002.

[24] Y. T. Didenko and K. S. Suslick. The energy efficiency of formation of photons, radicals and ions during single-bubble cavitation. Nature, 418 (6896):394-397, 2002. 
[25] M. W. A. Kuijpers, M. F. Kemmere, and J. T. F. Keurentjes. Calorimetric study of the energy efficiency for ultrasound-induced radical formation. Ultrasonics, 40(1-8):675-678, 2002.

[26] A. S. D. Rochebrochard, J. F. Blais, and E. Naffrechoux. Comparison of characterization methods in high frequency sonochemical reactors of differing configurations. Ultrason. Sonochem., 17(3):547-554, 2010.

[27] V. S. Sutkar and P. R. Gogate. Design aspects of sonochemical reactors: Techniques for understanding cavitational activity distribution and effect of operating parameters. Chem. Eng. J., 155(1-2):26-36, 2009.

[28] L. Hallez, F. Touyeras, J. Y. Hihn, J. Klima, J. L. Guey, M. Spajer, and $Y$. Bailly. Characterization of hifu transducers designed for sonochemistry application: Cavitation distribution and quantification. Ultrasonics, 50(2): 310-317, 2010.

[29] G. Mark, A. Tauber, L. A. Rudiger, H. P. Schuchmann, D. Schulz, A. Mues, and $\mathrm{C}$. von Sonntag. OH-radical formation by ultrasound in aqueous solution - Part II: Terephthalate and Fricke dosimetry and the influence of various conditions on the sonolytic yield. Ultrason. Sonochem., 5(2):4152, 1998.

[30] A. Mandroyan, R. Viennet, Y. Bailly, M. L. Doche, and J. Y. Hihn. Modification of the ultrasound induced activity by the presence of an electrode in a sonoreactor working at two low frequencies (20 and $40 \mathrm{kHz}$ ). Part I: Active zone visualization by laser tomography. Ultrason. Sonochem., 16 (1):88-96, 2009. 


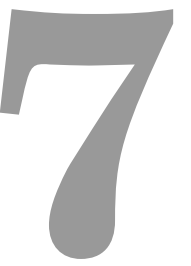

\section{High-speed imaging of $1 \mathrm{MHz}$ surface acoustic cavitation ${ }^{1}$}

The effectiveness of ultrasonic and megasonic cleaning is related to the dynamics of acoustically driven microbubbles in close proximity to the liquid-solid interface. Here the dynamics of a cleaning cavitation bubble is studied using combined visualization of both particle removal and bubble dynamics. Observations of stable asymmetric bubble oscillations suggest a possible mechanism for particle removal through water jet impact onto the substrate.

\subsection{Introduction}

Since the introduction of megasonic cleaning in semiconductor industry in the early 80 's of the last century, a debate has been going on about which physical mechanism is responsible for the removal of particles. Because of the high frequencies involved $(>700 \mathrm{kHz})$ it was believed that acoustic cavitation could not occur and cleaning was attributed to phenomena such as Eckart and Schlichting streaming or pressure build-up on particles [1, 2]. Recently it was shown however, that the removal of nanoparticles is closely related to the presence of acoustic cavitation in megasonic cleaning systems [3, 4]. The dependence of particle removal efficiency on the concentration of dissolved gas and the presence of sonoluminescence and surface cavitation bubbles are clear indications that the underlying mechanism is related to bubble dynamics.

As the requirements for cleaning in semiconductor processing are ever more stringent, it becomes necessary to obtain a thorough understanding of the physical behavior of acoustically driven microbubbles in contact with a solid wall.

\footnotetext{
${ }^{1}$ Based on: Aaldert G. Zijlstra, Tom Janssens, Kurt Wostyn, Michel Versluis, Paul M. Mertens and Detlef Lohse, High speed imaging of $1 \mathrm{MHz}$ driven microbubbles in contact with a rigid wall, Solid State Phenomena Vols. 145-146 (2009) pp 7-10
} 
In particular, the forces exerted thereby which might clean or damage a substrate are of interest. It is generally understood that the cleaning effect of ultrasonic and megasonic cleaning is based on large and local fluid velocities and stresses arising from acoustically induced bubble activity in the vicinity of an object. However, whereas a fairly extended amount of literature exists on acoustic cavitation in the bulk of the liquid and transient laser generated bubble collapse near an interface, the opposite is true for the behavior of acoustically driven bubbles in contact with a solid interface. Here, we address this problem by visualization of both the removal of nanoparticles and the sub-microsecond timescale dynamics of the cavitation bubbles responsible thereof.

\subsection{Experimental setup}

\subsubsection{The acoustic field}

The main challenge in this experiment is to simultaneously visualize the nanoparticle removal and the microbubble dynamics. The difficulty arises from the fact that bubbles in an acoustic field are microscopically small, very mobile and short lived. Options to overcome these problems are e.g. using bubbles stabilized against dissolution (ultrasound contrast agents) [5, 6], bubbles attached to the wall by capillary forces [7, 8] or entrapped surface bubbles (see previous chapters of this thesis). However, these methods can not be used here since our aim is to study naturally occurring cavitation bubbles of which the dynamics results in the cleaning of a substrate. In order to increase the probability of locating and imaging such a cleaning bubble the setup shown in Fig. 7.1 is used. The experimental megasonic cleaning device consists of a rectangular acrylic glass container with inner dimensions $18 \times 8 \times 6 \mathrm{~cm}^{3}$, filled with demineralized water (ambient conditions). The ultrasound field is introduced by means of a submersible $1056 \mathrm{kHz}$ piezoelectric transducer (Sonosys). In this configuration, the reflection of the sound from the far-end-wall back to the transducer creates a standing-wave field with a wavelength of $1.5 \mathrm{~mm}$. A generic feature of standing acoustic fields is that bubbles migrate towards either the pressure node or antinode under the influence of primary Bjerknes forces [9]. This standing field thus reduces the randomness of the bubble distribution thereby increasing the chances to image a cleaning bubble. 


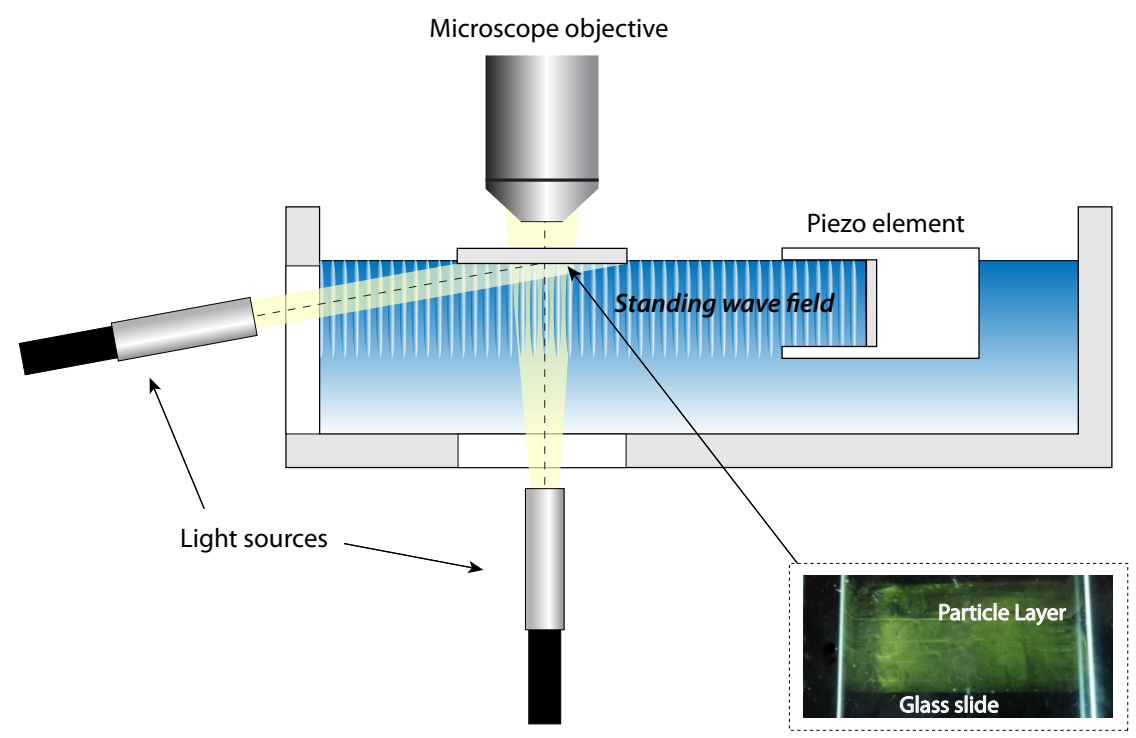

Figure 7.1: Experimental setup (side view) showing the visualization methods for (i) the gold particle removal with continuous illumination under an angle (dark-field) and (ii) the high speed imaging of the single bubble using a bright short duration light flash from below. Inset shows an image of the microscope slide holding a layer of gold nanoparticles.

\subsubsection{The particle layer}

For visualization purposes the substrate which is subjected to the cleaning process is required to be transparent, therefore a standard glass microscope slide $\left(74 \times 26 \times 1 \mathrm{~mm}^{3}\right.$, Menzel-Gläzer $)$ was chosen, cleaned ultrasonically beforehand in ethanol. On one side, a layer of gold nanoparticles $(100 \mathrm{~nm}$ colloid, Ted Pella Inc.) is deposited using a method adapted from Malaquin et al. [10] which is based on the principle of convective assembly. Here, a drop of gold nanoparticle suspension is placed between two microscope slides $0.5 \mathrm{~mm}$ apart. The top slide is then translated with a velocity of $5 \mu \mathrm{m} / \mathrm{s}$ parallel to the lower slide on which the particles are deposited. A closely packed particle layer is created with a total area of approximately $2 \mathrm{~cm}^{2}$ (see inset Figure 7.1). The use of gold nanoparticles was motivated by two reasons: (i) their nanoscopic size makes them representative for the particles encountered on actual semiconductor substrates and (ii) due to the enhanced surface plasmon resonance [11], the particles are very efficient scatterers of visible light (optimum lies around $530 \mathrm{~nm}$ ). The latter property is crucial for the experiment since nanoscopic 
particles are normally undetectable with standard optical methods. The scattered light allows visual observation of the particle layer thereby providing an excellent medium for in-situ monitoring of the cleaning process.

The microscope slide with the particle layer facing downward was placed parallel to, and in contact with the water-air interface (Fig. 7.1). In this configuration, the pressure (anti)nodal planes and therefore the planes of cavitation activity are perpendicular to the glass surface. Placement of the slide in this manner greatly simplifies optical access and circumvents the necessity of placing an intrusive microscope objective within the acoustic field. Additionally, distortion due to motion of the free surface is avoided.

\subsubsection{Imaging methods}

The timescale of the cleaning process is measured in seconds while the individual bubble dynamics is dictated by the period of the acoustic field which in this case is $1 \mu \mathrm{s}$. Hence in order to capture the cleaning process it suffices to take images of the particle layer every second or so. However, to resolve the bubble dynamics at less than microsecond resolution the use of a high speed imaging system called Brandaris 128 [12] was required. This camera is based on the rotating mirror principle and capable of recording 128 frames with framerates up to $25 \mathrm{Mfps}$. Both camera's were connected to the microscope system enabling the recording of both the particle removal and the bubble dynamics. Image magnification of the particle layer and the cavitation bubbles was provided by a microscope objective (Olympus, $10 \times$ PlanC N, NA 0.25, WD $10.5 \mathrm{~mm}$ ) connected to an Olympus microscope with an additional internal $2 x$ image magnification. This magnification was chosen to obtain a reasonably sized field-of-view $\left(520 \times 380 \mu \mathrm{m}^{2}\right)$ which is needed to increase the likelihood of capturing a cleaning bubble, but at the same time achieve sufficient optical resolution to resolve the bubble dynamics. The scattered light originating from the particle layer is of low intensity thus the use of dark field illumination (grazing angle, continuous lightsource Olympus ILP-1) and camera exposure times (single frame CCD-camera, Pixelink 741A) of typically $100 \mathrm{~ms}$ were required.

For the high-speed recordings, a bright and short duration light pulse $(200 \mu \mathrm{s}$, Perkin Elmer MVS-7010) was applied through the lower viewport of the liquid container. In these recordings the scattered light from the gold nanoparticle layer is not visible as it is flooded by this much brighter backlighting. Ideally, the framerate should be as high as possible to capture the rapid motion of the bubble oscillations, however to cover sufficient acoustic periods and in order to obtain a reasonable image contrast (see the discussion in section 3.2) a framerate of around $12 \mathrm{Mfps}$ was used. 


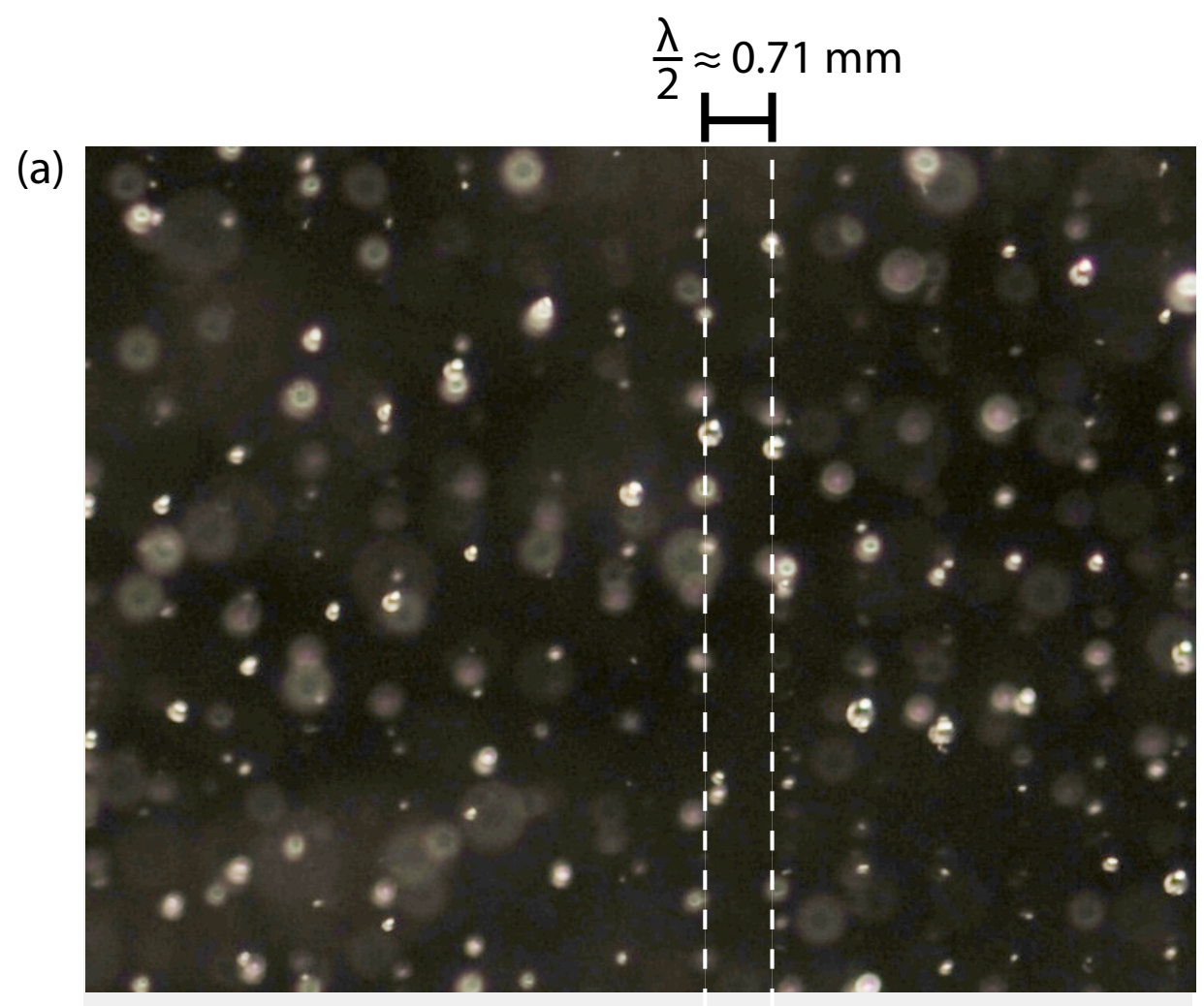

(b)

Figure 7.2: (a) Sideview of a section of a fully developed bubble field. Note that in this image only the larger bubbles can be distinguished (between 50 and $300 \mu \mathrm{m}$ in diameter) which are located in the nodal planes of the acoustic field. (b) Intensity plot of the summed pixelvalues of the image in (a) along the vertical direction illustrating the periodicity of the bubble locations in the standing wave field.

An experiment consisted of locating the regions of cleaning activity using a first glass slide with a particle layer exposed to the acoustic cavitation. This way the imaging system could be focused on one of these sections thereby increasing the probability of imaging a cleaning event. Next, the slide was replaced and the sound field was reactivated while monitoring and recording the particle layer. Whenever a cleaning bubble appeared in the field-of-view, the high-speed camera was triggered. 


\subsection{Results}

\subsubsection{Particle removal}

The driving amplitude of the transducer was set to a value above the nucleation threshold of acoustic cavitation (acoustic pressures between $200-300 \mathrm{kPa}$, estimated using a hydrophone, Onda 500-HNR). Following activation of the acoustic field it was observed that bubbles first appeared in the region close to the transducer and subsequently also the consecutive more distant nodal planes were populated. After several minutes the bubble field extended from the transducer surface to the back wall of the container. Part of the developed bubble field is shown in Figure 7.2. Interestingly, the start of observable gold nanoparticle removal was found to coincide with the first appearance of cavitation bubbles in the planes directly below the glass slide.

This "buildup-time" of the acoustic field is also reflected in the graph of the cleaned area in time of a small monitored section of the substrate (Figure 7.3). Only after about 100 seconds the cavitation bubble field extended to this particular section some $3 \mathrm{~cm}$ away from the transducer surface. As shown in the 4 successive images of Figure 7.3(a), a patch of $700 \times 100 \mu \mathrm{m}^{2}$ (dark region) is cleaned in a time frame of 60 seconds. Clearly visible in these images are bright spots, resulting from the scattered light originating from a single bubble (as will be shown later) moving rapidly adjacent to the substrate. These spots have a blurry and smeared out appearance owing to the relative long exposure time of $100 \mathrm{~ms}$ compared to the typical timescale of the bubble motion. These and similar images reveal that locations of particle removal coincide with the locations traversed by the bubble. This area is oriented along the line of intersection of the glass surface and the nodal planes of the acoustic field. The position of the nodal planes was verified using a hydrophone. The bubble motion appears quite erratic, displaying rapid translations (e.g. see the blur in frame (iii)), followed by brief periods without significant displacement (like in frame (ii)). Based on the locations of the bright spots, the bubble appears to be positioned mostly at the right hand side of the line of intersection, which is the side closest to the transducer. Since the motion of bubbles is determined by Bjerknes forces, this preferred position as well as the erratic motion are likely to result from fluctuations in the acoustic field or the bubble size.

Following the initiation of the acoustic field and the appearance of cleaning bubbles, the rate of particle removal was observed to diminish (as demonstrated by the flattening of the graph of Figure 7.3). Initially this was related to the confined areas of surface bubble activity where all particles were already removed. Later on, however, also the bubble activity subsided, leaving only relatively immobile and larger sized bubbles attached to the substrate. 

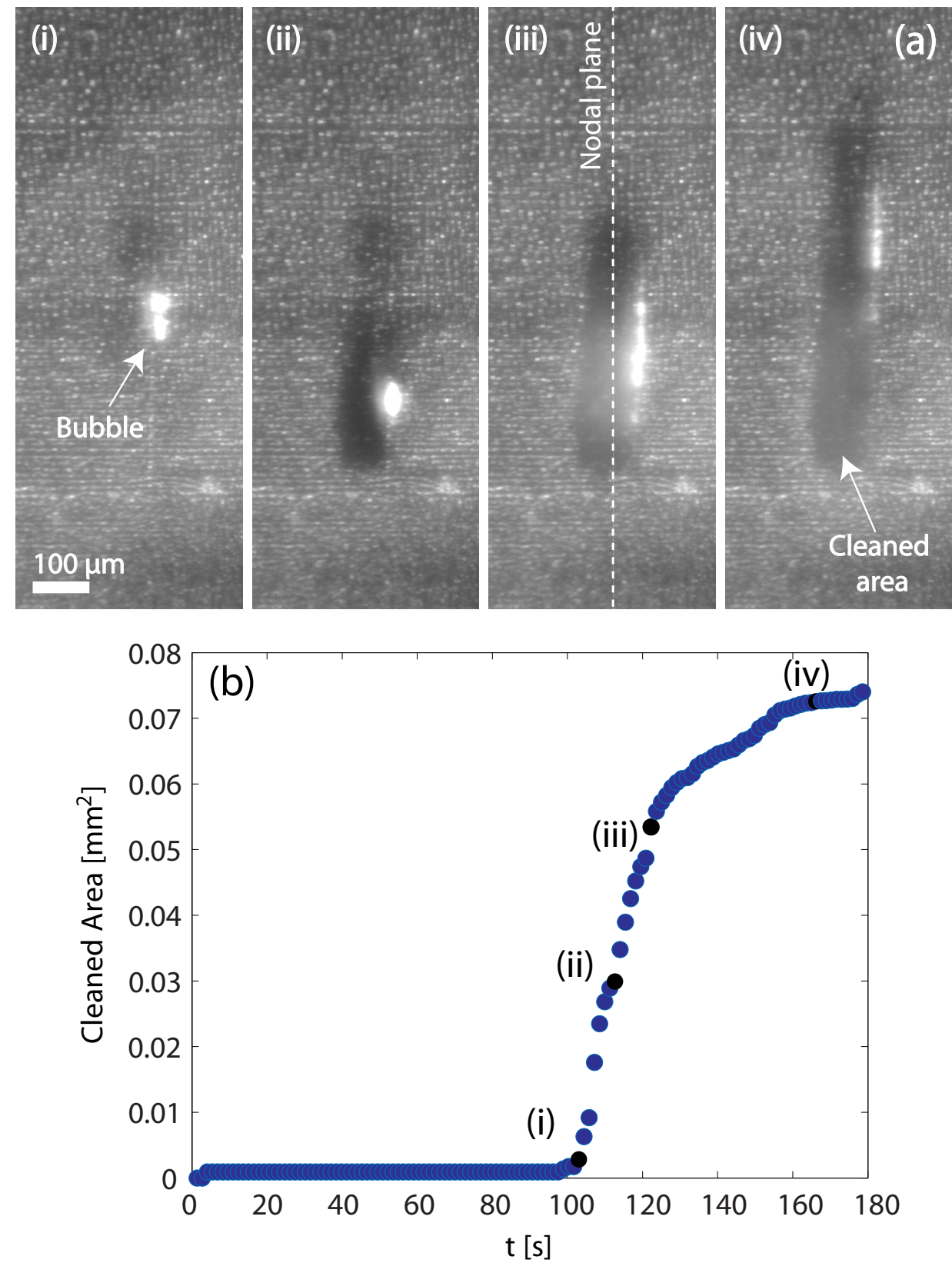

Figure 7.3: (a) Plan-view light scattering images showing the removal process of a layer of $100 \mathrm{~nm}$ gold particles from a glass substrate. The patchy grayish area is the particle layer. The dark regions are sections where the particles are removed. The bright spots originate from a bubble moving close to - or on the substrate. The horizontal dashed line in frame (iii) marks the approximate intersection of a nodal plane with the substrate. (b) Graph of the cleaned area in which the frames of (a) are indicated. 

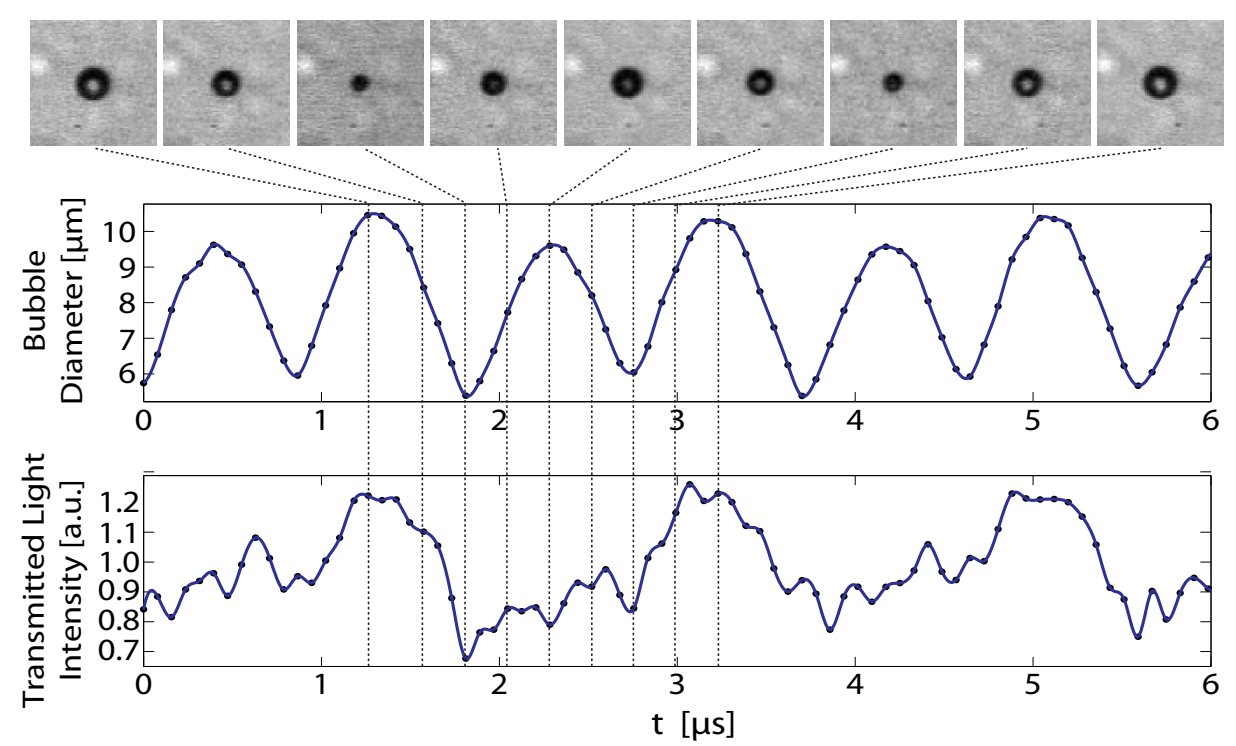

Figure 7.4: Top view of the "cleaning bubble". Shown in the graphs are the bubble diameter obtained from the high-speed movie and the transmitted light in the center of the bubble.

\subsubsection{Cleaning bubble dynamics}

In order to also capture the sub-microsecond timescale dynamics of the bubbles responsible for cleaning of the substrate, recordings were made using the Brandaris high speed camera. A first striking result was that all of the obtained 24 recordings of 4 separate cleaning events displayed only a single radially oscillating microbubble. Additionally, the oscillations are stable and continue for many consecutive cycles. This observation is in contrast with the common idea that cavitation bubbles near surfaces are transient, that is, lasting only a few cycles before fragmenting or dissolving.

Nine selected frames from such a high speed movie recorded at $12.8 \mathrm{Mfps}$ are depicted in Figure 7.4 showing bubble oscillations in top-view during two acoustic cycles. From this perspective, the bubble appears to remain spherical. The diameter of the bubble was extracted using image processing techniques implemented in Matlab and a typical result is shown in the top graph of Figure 7.4. The bubble with a mean diameter of $8 \mu \mathrm{m}$ oscillates at the fundamental driving frequency of $1 \mathrm{MHz}$, yet contains a significant subharmonic component as is apparent from the radial oscillation amplitude which is alternating between 1 and $1.5 \mu \mathrm{m}$ (about 25-40\%) with every cycle. 
In principle, top-view imaging only allows observation of the lateral bubble dynamics. However, inspection of the region within the bubble perimeter does provide some clues about the bubble shape in axial direction. As shown in Figure 7.4 a bright circular shape can be distinguished centered within the bubble measuring approximately one-third of the bubble diameter. These shapes are not uncommon in images of spherical bubbles and result from light refraction at the liquid-gas interfaces (see e.g. Figure 6 in [13]). If this bubble would remain spherical it is expected that the transmitted light intensity follows the same trend as the bubble radius, but as shown in the lower graph of Figure 7.4 this is not the case. Here the transmitted light intensity, measured from the pixel values within the circular shape, shows a periodicity which is half the frequency of the driving acoustic field where the maximum coincides with the maximum bubble diameter. The most plausible explanation is that the bubble deviates from sphericity which is well known behavior for acoustically driven bubbles in close proximity with a solid substrate [14, 15].

The experimental setup used here was designed for top-view observations, nevertheless imaging of cavitation activity on the edge of the glass substrate did result in an illustrative recording which supports this explanation (Figure 7.5). The 40 frames depicted here correspond to three acoustic cycles starting at $t=0$ where the base of the hemispherical bubble is $13 \mu \mathrm{m}$ in width. The graph of the measured bubble width as shown in figure 7.6 demonstrates a qualitative similar result as the diameter-time curve of the bubble obtained from the recording in top view (Figure 7.4). Again, a subharmonic component is present. In these frames the bubble displays hemispherical collapse (e.g. from $\mathrm{t}=0 \mu \mathrm{s}$ to $\mathrm{t}=0.39 \mu \mathrm{s})$ after which the bubble expands with what appears to be an indentation in the upper bubble wall. During this stage (e.g. from $t=0.47$ $\mu \mathrm{s}$ to $\mathrm{t}=1.02 \mu \mathrm{s})$ the bubble is wider and less elevated than in the previous cycle. The same process is repeated in the following cycles, giving rise to the subharmonic component in the graph of Figure 7.6.

The indentation may be explained by a repetitive splitting of the bubble, but a more likely explanation is that a liquid jet is formed during the hemispherical collapse (somewhere between $t=0.32 \mu \mathrm{s}$ and $t=0.39 \mu \mathrm{s}$ ). This jet flow is an extreme manifestation of non-spherical bubble oscillation and may induce liquid flow directed through the center of the bubble which persists during the bubble expansion phase. The result is a toroidal shaped bubble. Indeed, in the frames in $\mathrm{t}=1.02 \mu \mathrm{s}$ or $\mathrm{t}=2.99 \mu \mathrm{s}$ a small column of liquid measuring approximately 1 $\mu \mathrm{m}$ in diameter can be distinguished. This same water column may also explain the occurrence of the bright patch observed in the top-view recordings. 


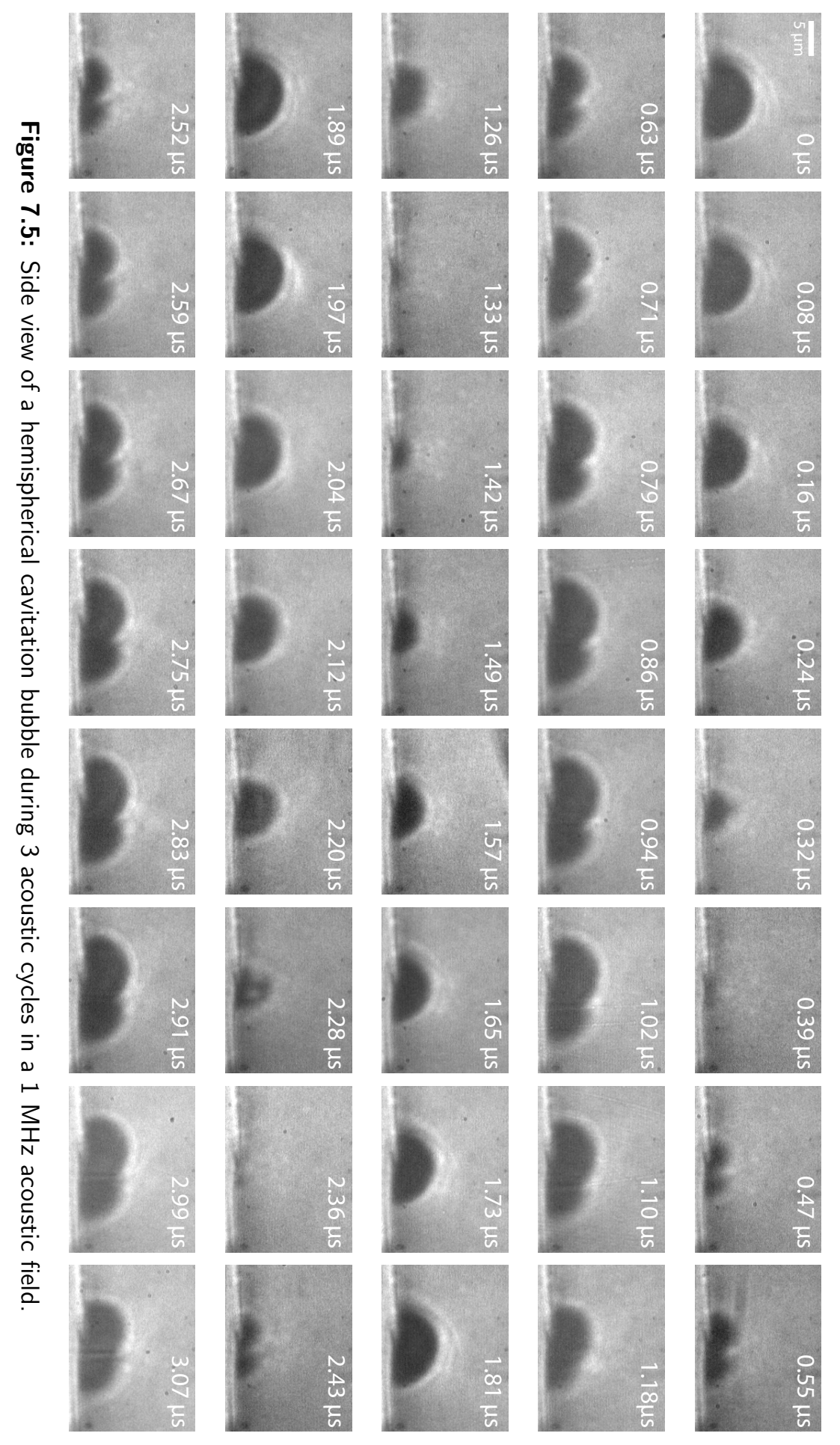




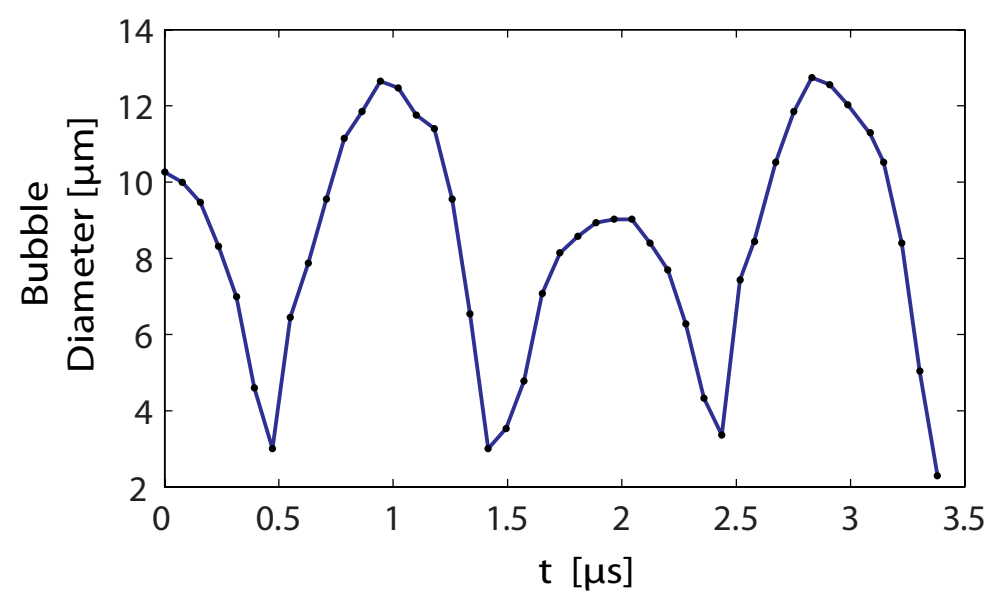

Figure 7.6: Diameter of the base of the bubble shown in Figure 7.5

\subsection{Discussion and conclusion}

With the combined visualization of both particle removal and bubble dynamics the prominent involvement of acoustic cavitation in the $\mathrm{MHz}$ regime in the removal of nanoparticles was demonstrated. The recordings reveal that in the standing wave configuration, where the solid surface is oriented perpendicularly to the acoustic field, a single bubble is responsible for the cleaning effect. This configuration is commonly found in batch-type megasonic cleaning devices. In the experiment the cleaning efficiency is rather low however, since the bubble remained confined to small areas oriented along the intersection lines of the nodal planes. Also the growth and accumulation of larger bubbles is likely to have a negative effect on the cleaning activity e.g. due to acoustic shielding effects. This may be remedied with the use of continuous liquid flow which serves to remove these larger bubbles and sustain constant conditions.

Evidently, the dynamics of the bubble induces localized liquid motion which generates the forces required to remove particles from the substrate. The dynamics of the cleaning bubbles observed in top-view in the experiment (Figure 7.4) is characterized by considerable radial oscillations with a subharmonic component. The therewith related transmitted light through the bubble suggest that it may also display non-spherical shape oscillations. This assumption is backed up by the observation of non-spherical bubble oscillations.

Now the question is: what are the forces exerted on the particles and the substrate and how are these related to the bubble motion? The measurement of these forces is extremely complicated due to the microscopic time and length scales involved. Nevertheless some estimates for the normal and shear 
stress have recently been obtained by Vos et al. [15] using experimentally obtained (contrast agent) microbubble response curves as an input for a parametric model. Here, shear stresses between 25-350 kPa were found, occurring during the bubble compression phase. In addition, normal forces were estimated to be up to 5 times higher than the driving pressure (almost $1 \mathrm{MPa}$ ). Even though these bubbles were stabilized by a phospholipid coating and smaller than the cavitation bubbles encountered in the current study, the similarities are evident. Both the subharmonic component as well as shape deformations are observed, hence similar values for the induced forces may be associated with the cleaning bubble.

Even larger forces may occur in the event of jet formation to which cleaning and surface damage are commonly attributed. Based on the displacement of the top of the bubble wall (Figure 7.5) between e.g. $t=0.32 \mu$ s to $t=0.39 \mu \mathrm{s}$ it can be inferred that this section of the bubble wall attains a velocity of at least $40 \mathrm{~m} / \mathrm{s}$ towards the substrate. The impact of the liquid onto the substrate will be accompanied by strong normal forces and possibly also shear forces due to radial spreading boundary flow. As argued by Ohl et. al [16], the latter is likely to be the most prominent boundary flow and therefore responsible for particle removal. Further study is required however to explore the dynamics of cavitation bubbles in close proximity of a solid surface and to establish the relation between this dynamics and the induced forces thereof. 


\section{Bibliography}

[1] A. Busnaina and T. Elsawy. Post-cmp cleaning using acoustic streaming. J. Electron. Mater., 27:1095-1098, 1998.

[2] M. Olim. A theoretical evaluation of megasonic cleaning for submicron particles. J. Electrochem. Soc., 144(10):3657-3659, 1997.

[3] G. Vereecke, F. Holsteyns, S. Arnauts, S. Beckx, P. Jaenen, K. Kenis, M. Lismont, M. Lux, R. Vos, J. Snow, and P. W. Mertens. Evaluation of Megasonic Cleaning for Sub-90nm Technologies. Solid State Phenomena, 7(103-104):141-146, 2005.

[4] W. Kim, T. H. Kim, J Choi, and H. Y. Kim. Mechanism of particle removal by megasonic waves. Appl. Phys. Lett., 94(8):081908-3, 2009.

[5] H. J. Vos, B. Dollet, J. G. Bosch, M. Versluis, and N. de Jong. Nonspherical vibrations of microbubbles in contact with a wall-a pilot study at low mechanical index. Ultrasound Med. Biol., 34(4):685-688, 2008.

[6] P. Prentice, A. Cuschieri, K. Dholakia, M. Prausnitz, and P. Campbell. Membrane disruption by optically controlled microbubble cavitation. Nature Physics, 1:107-110, 2005.

[7] S. Nakatani, K. Yoshida, and Y. Watanabe. Optical and acoustic observations of bubble adhered to piezoelectric transducer under ultrasound field: Pressure signal caused by bubble behavior. Jpn. J. Appl. Phys., 47(5): 3904-3907, 2008.

[8] F. Prabowo and C. D. Ohl. Surface oscillation and jetting from surface attached acoustic driven bubble. Ultrason. Sonochem., 18(1):431 - 435, 2011.

[9] A. A. Doinikov. Translational motion of a spherical bubble in an acoustic standing wave of high intensity. Physics of Fluids, 14(4):1420-1425, 2002.

[10] L. Malaquin, T. Kraus, H. Schmid, E. Delamarche, and H. Wolf. Controlled particle placement through convective and capillary assembly. Langmuir, 23(23):11513-11521, 2007.

[11] P. K. Jain, K. S. Lee, I. H. El-Sayed, and M. A. El-Sayed. Calculated absorption and scattering properties of gold nanoparticles of different size, shape, and composition: Applications in biological imaging and biomedicine. The Journal of Physical Chemistry B, 110(14):7238-7248, 2006. 
[12] C. T. Chin, C. Lancée, J. Borsboom, F. Mastik, M. E. Frijlink, N. de Jong, M. Versluis, and D. Lohse. Brandaris 128: A digital 25 million frames per second camera with 128 highly sensitive frames. Rev. Sci. Instr., 74(12): 5026-5034, 2003.

[13] W. Lauterborn, T. Kurz, R. Geisler, D. Schanz, and O. Lindau. Acoustic cavitation, bubble dynamics and sonoluminescence. Ultrason. Sonochem., 14(4):484 - 491, 2007.

[14] J. R. Blake and D. C. Gibson. Cavitation bubbles near boundaries. Ann. Rev. Fluid Mech., 19(1):99-123, 1987.

[15] H. J. Vos, B. Dollet, M. Versluis, and N. de Jong. Nonspherical shape oscillations of coated microbubbles in contact with a wall. Ultrasound Med. Biol., 37(6):935 - 948, 2011.

[16] C. D. Ohl, M. Arora, R. J. Dijkink, V. Janve, and D. Lohse. Surface cleaning from laser-induced cavitation bubbles. Appl. Phys. Lett., 89(7): 074102, 2006. 


\section{8}

\section{Conclusions and outlook}

Most applications of acoustic cavitation involve the interaction of bubbles with a solid surface. In addition, cavitation activity is usually believed to originate from bubble nuclei stabilized inside crevices which are present in almost any surface. Even though these particular aspects of acoustic cavitation are of fundamental as well as practical importance, the existing literature on both topics is surprisingly limited. This is most likely caused by the difficulty of the task to locate the bubbles and to resolve the associated microscopic time- and lengthscales

In this thesis three techniques are described and applied enabling the study of surface acoustic cavitation. First of all, micropits which are micromachined in a silicon surface constitute model crevices. The precise control over their dimensions and locations allows detailed investigation of the initiation of cavitation activity and subsequently also of cavitation bubble interaction with the interface and its effects (chapters 5 and 6 ). The second method to obtain improved control over the location of cavitation bubbles is by means of a standing-wave field (chapter 7). And finally, the use of a novel ultrashort illumination technique permits high-resolution imaging without motion-induced blur of cavitation bubbles (chapter 3).

An essential feature of such an entrapped bubble is its exceptional stability against dissolution. In chapter 2, theoretical investigation suggests that there is an upper limit of the radius of a micropit, below which the bubble is in stable equilibrium in a stagnant liquid and that this limit is sensitive to the amount of gas dissolved in the liquid. When driven by ultrasound such a stable micropit bubble will respond by expansion and contraction. In the second part of chapter 2, this behavior was explored for small oscillation amplitudes. In the resulting axisymmetric linear model based on the unsteady Stokes equation and a no-slip boundary condition, the vibration of the liquid-gas interface is similar to a classic damped oscillator. In addition, the model predicts that the interface exhibits higher order surface modes with increasing frequency. These surface 
modes might be a precursor for the destabilization of the interface leading to bubble pinch-off and the subsequent cavitation inception. This assumption may be tested by means of stability analysis combined with vibrometer measurements or high-speed imaging.

Illumination by high-intensity laser induced fluorescence enables ultrashort flash photography for high-resolution imaging on a nanoseconds timescale (chapter 3). The intensity of the light obtained with this method is one order of magnitude larger than that of several other state-of-the-art light-sources. This makes it highly suited for accurate imaging without motion-induced blur of fast and small scale phenomena such as inkjet droplets but also acoustic cavitation bubbles. Further improvement of the technique may be achieved by optimizing the components of the illumination system.

Using this method of illumination, it was shown that the pressure trace of a shock wave measured using a fiber optic probe hydrophone consists of a superposition of the reproducible direct wave with random secondary waves emitted by collapsing bubbles in the vicinity of the probe (chapter 4). Furthermore, adding acetic acid results in significant decrease of the number of bubbles and therewith the secondary shock waves and their contribution to the hydrophone signal.

Above a pressure threshold, acoustic actuation of an entrapped cylindrical microbubble results in shedding of microbubbles from its liquid-gas interface into the liquid (chapter 5). At $200 \mathrm{kHz}$ driving frequency the generated microbubbles respond by strong expansion and contractions while their interactions result in the formation of a hemispherical bubble cloud localized on the micropit. This demonstrates that the introduction of artificial crevice bubbles significantly enhances cavitation activity since no cavitation is observed in its absence. Additional micropits further increase this activity where the neighboring cavitation clouds interact and merge. It is concluded that the loss of gas from the intermittently pinched-off bubbles must be continuously replenished by diffusive transport considering the remarkable stability of the process (it can last for hours).

Cavitation promoted by the presence of artificial crevice bubbles provides unique conditions not only for detailed investigation of surface acoustic cavitation and its effects such as shock waves and surface damage, but also for enhancing the efficiency of various applications. Further study is required to investigate the exact mechanism of bubble pinch-off and the conditions for which this occurs.

Enhancing cavitation activity by means of introducing micropit bubbles in an insonified liquid likewise enhances the sonochemical production of hydroxyl $(\mathrm{OH})$ radicals (chapter 6$)$. This is demonstrated quantitatively by teraphtalic 
acid dosimetry. Higher production rates can be obtained by increasing the acoustic driving amplitude or the number or the number of pits. The localized character of the micropit enhanced cavitation combined with the high resolution imaging method as described in chapter 3 may be used to establish a correlation between the bubble population and the sonochemical yield.

In chapter 7 another combination of cavitation bubble localization with highspeed imaging methods was employed. Here it was shown that particle removal in a $1 \mathrm{MHz}$ standing-wave field configuration occurs along regions near the intersection lines of the nodal planes with the substrate. In addition, it was shown that particle removal results from the forces induced by stable oscillations of a single cavitation microbubble moving adjacent to the solid surface. The radial oscillations (observed in top-view) of these microbubbles contain a subharmonic component which are likely due to asymmetrical bubble oscillations.

As demonstrated and discussed in this thesis, increased localization of cavitation activity by means of the introduction of artificial micropit bubbles or standing-wave fields not only greatly facilitates the study of surface acoustic cavitation, but perhaps also benefits its numerous applications. 
Merely the presence of compressible entities, known as bubbles, greatly enriches the physical phenomena encountered when introducing ultrasound in a liquid. Mediated by the response of these bubbles, the otherwise diffuse and relatively low energy density of the acoustic field can induce strong, localized liquid motion, high internal temperatures and pressures as well as secondary acoustic emissions. In turn, these effects give rise to considerable stresses exerted on nearby objects and molecular dissociation of the bubble constituents. These unique characteristics of acoustic cavitation enable a wide variety of applications, notably ultrasonic cleaning and sonochemistry.

Scientific knowledge of cavitation bubbles predominantly comprises the dynamics and effects of pre-existing spherical bubbles in an infinite liquid medium. Much less is known about the origin of cavitation bubbles or their behavior when in close proximity to a solid surface. A disparity that can be attributed to the experimental difficulties posed by the microscopic length- and timescales combined with the rapid and unpredictable motion which characterizes cavitation bubbles. Still, for successful application of acoustically driven bubbles, thorough understanding of their coming into existence as well as their interaction with solid surfaces is of great importance.

In this thesis both these aspects are investigated. An emphasis is placed on the role of microbubbles entrapped inside artificially created micropits. This is motivated by two reasons. First of all, acoustic cavitation bubbles are believed to originate mostly from stable microscopic volumes of gas entrapped in naturally occurring crevices inside a solid object. An artificially created micropit thus constitutes a model for such a crevice. Secondly, modern micromachining techniques enable precise control over the dimensions and locations of such micropits. This greatly facilitates the experimental study of acoustically driven micropit bubbles and the various aspects of the therewith induced surface acoustic cavitation.

In contrast to a free floating bubble, a micropit bubble can be stable against dissolution in a stagnant liquid. In the first part of chapter 2, this stability is theoretically investigated for a cylindrical micropit. It follows that there is an upper and lower limit for the gas concentration between which stability is ensured. In addition, these limits depend on the radius of the micropit where a smaller radius implies a larger range.

The response of such a stable cylindrical micropit bubble to an acoustic field is theoretically investigated in the second part of chapter 2. Under the condition of small amplitude interface excursions, the unsteady Stokes equation applies for which a solution is available. The shape and amplitude of oscillation of 
the axisymmetric liquid-gas interface are approximated with a Fourier-Bessel series expansion. The values for the coefficients of the series are obtained from numerically solving the equation which results from application of the dynamic and kinematic boundary conditions. The behavior of the liquid-gas interface is similar to the classic damped harmonic oscillator with the added feature of surface mode oscillations. These surface modes, which depend on the driving frequency and pit dimensions may be precursors to high amplitude capillary waves leading to bubble pinch-off.

High-speed imaging is a powerful tool for the study of fast and small scale objects such as acoustic cavitation bubbles. Accurate high-resolution images of these objects, that is without motion-induced blur, can be obtained by means of a short duration and sufficiently intense illumination pulse. In chapter 3 , a technique to generate such a pulse is introduced which is based on laser induced fluorescence. With this method, the coherence of the laser which is highly undesirable for imaging purposes is greatly reduced while maintaining a short pulse duration and high intensity.

The effectiveness of this illumination method is demonstrated in chapter 4. Here, the origin of the large variations which are present in the signal of a fiber optic probe hydrophone when measuring the pressure in a shock wave is investigated. The images reveal the occurrence of secondary shock waves resulting from cavitation bubble collapse. In the situation where cavitation activity is suppressed by adding acetic acid to the liquid, the signal variations are greatly reduced. This suggests that the secondary shock waves are the main cause for these variations.

The means to investigate if and how cavitation activity can be initiated from an artificial crevice bubble are provided by the precise control over its dimensions and location combined with the available high-speed imaging techniques. In chapter 5 , the response of a $30 \mu \mathrm{m}$ diameter micropit bubble to an $80 \mathrm{kHz}$ and $200 \mathrm{kHz}$ acoustic field is investigated. It is found that, above a pressure threshold, not only are microbubbles shed from the liquid-gas interface, it is also a remarkably stable process. Bubble pinch-off does not involve nucleation but appears to result from large amplitude capillary waves which locally fold the interface into a singularity. The stability of the microbubble generation process requires a continuous diffusive transport of gas back into the micropit bubble.

Of both frequencies the response of the generated bubbles to the acoustic field is most pronounced at $200 \mathrm{kHz}$, resulting in a hemispherical cavitation cloud centered on the micropit. Cavitation activity is further enhanced by introducing additional micropit bubbles. In this case, secondary Bjerknes forces cause neighboring cavitation bubble clouds to interact and form stable ribbon-like cavitation structures. An additional advantage of the enhanced and localized 
cavitation activity is that it enables detailed study of surface acoustic cavitation and its effects such as shock wave emission, damage formation but also sonochemical reactions.

Sonochemistry is a promising application of cavitation but requires significant increase in production yields if it is to become of widespread use. In chapter 6 , it is investigated if micropit bubble enhanced cavitation also results in increased sonochemical efficiency. The production rate of $\mathrm{OH}$ radicals by cavitation bubble collapse induced sonolysis, is quantified by means of terephtalic acid dosimetry. The results reveal that the sonochemical efficiency indeed increases by at least an order of magnitude compared to the situation without micropits. In addition, images reveal that luminescence, resulting from the reaction of luminol with $\mathrm{OH}$ radicals, originates from the regions of cavitation activity.

One of the most established applications based on surface acoustic cavitation is megasonic cleaning used for the cleaning of semiconductor substrates. However the continuing downsizing of the fragile structures makes efficient and damage-free cleaning of these substrates extremely challenging. In chapter 7 , the surface acoustic cavitation bubbles which may clean or damage are investigated. Locating of these bubbles is achieved by means of a layer of gold nanoparticles deposited on a transparant microscope slide which is placed perpendicular and on top of a $1 \mathrm{MHz}$ standing-wave field. Both the long timescale particle removal as well as the sub-microsecond dynamics of the bubbles responsible thereof are recorded in top-view. The results show that in this configuration, cleaning occurs in patches aligned with the nodal planes. In addition, particle removal is achieved due to forces exerted by single bubbles moving rapidly and adjacent to the interface. Moreover, the radial oscillations of these bubbles contain a strong subharmonic component. This component may be attributed to asymmetrical bubble oscillations involving jet formation which is likely to induce the largest forces on the solid surface. 


\section{Samenvatting}

$\mathrm{Er}$ is niet meer nodig dan de aanwezigheid van de samendrukbare objecten, beter bekend als bellen, om het aantal fysische fenomenen die zich voordoen bij het introduceren van ultrageluid in een vloeistof sterk uit te breiden. De reactie van deze belletjes maakt het mogelijk dat het in de regel diffuse- en laag energetische akoestische veld sterke, gelokaliseerde vloeistof stromingen, hoge interne temperatuur en druk maar ook secundaire akoestische emissies kan induceren. Als gevolg hiervan worden aanzienlijke krachten uitgeoefend op nabijgelegen objecten en kan er moleculaire dissociatie plaatsvinden in de bel. Deze unieke eigenschappen van akoestische cavitatie maken een breed scala aan toepassingen mogelijk, in het bijzonder ultrasoon reinigen en sonochemie.

De wetenschappelijke kennis van cavitatie omhelst voornamelijk de dynamica en de effecten van reeds bestaande sferische belletjes in een oneindig en vloeibaar medium. Er is veel minder bekend over de herkomst van de belletjes of hun gedrag in de nabijheid van een vast oppervlak. Deze ongelijke verdeling kan worden toegeschreven aan de experimentele moeilijkheden die voortvloeien uit de kleine tijd- en lengteschalen, gecombineerd met de snelle en onvoorspelbare bewegingen die cavitatie bellen kenmerkt. Echter, voor een succesvolle toepassing van akoestisch aangedreven bellen is diepgaand inzicht in het ontstaan van deze bellen alsmede de interactie met een vaste oppervlak van groot belang.

In dit proefschrift zijn beide aspecten onderzocht waarin nadruk wordt gelegd op de rol van de microbellen die zijn ingesloten in kunstmatig gecreëerde microputjes. Dit heeft twee redenen. Ten eerste, over het algemeen wordt de oorsprong van akoestische cavitatiebellen voornamelijk toegeschreven aan een stabiel, microscopisch klein gas volume, gevangen is in een holte zoals die van nature voorkomen in het oppervlak een vast voorwerp. Een kunstmatig gecreëerde microput vormt hiervoor aldus een model. Ten tweede, moderne micro-bewerkingstechnieken maken het mogelijk om de afmetingen en locaties van deze microputjes nauwkeurig te controleren. Dit resulteert in een aanzienlijke vereenvoudiging van de experimentele studie van de akoestisch aangedreven microput-bel en de verscheidene aspecten van de daaruit geïnduceerde akoestische oppervlakte cavitatie.

In tegenstelling tot een vrije bel, zijn er toestanden waarvoor een bel in een microput stabiel blijft en niet oplost in een stilstaande vloeistof. In het eerste deel van hoofdstuk 2 is deze stabiliteit theoretisch onderzocht voor een cilindrische microput. Er volgt dat er een bovenste en onderste limiet bestaat voor de gas concentratie waartussen stabiliteit mogelijk is. Deze limieten worden bepaald door de straal van de microput waar een kleinere straal een groter bereik impliceert. 
De reactie van een dergelijke stabiele microput-bel op een akoestisch veld is theoretisch onderzocht in het tweede deel van hoofdstuk 2. Onder de voorwaarde van kleine uitwijking amplitudes van het vloeistof-gas oppervlak is de tijdsafhankelijke Stokes-vergelijking geldig waarvoor een oplossing beschikbaar is. De vorm en de amplitude van de oscillaties van het axiaalsymmetrische oppervlak worden beschreven door de coëfficiënten van een Fourier-Bessel reeks. De waarden voor deze coëfficiënten volgen uit de numeriek oplossing van de vergelijking die resulteert uit het toepassen van de dynamische en de kinematische grens voorwaarden. Het gedrag van het vloeistof-gas oppervlak is vergelijkbaar met de klassieke gedempte harmonische oscillator met de toevoeging van oppervlakte modes. Deze modes welke afhankelijk zijn van de aandrijf frequentie en microput dimensies, zijn mogelijk voorlopers van hoge amplitude capillaire golven welke kunnen opbreken in belletjes.

Stroboscopische fotografie is een krachtig hulpmiddel voor het bestuderen van snelle en kleinschalige objecten zoals akoestische cavitatie belletjes. Nauwkeurige hoge-resolutie afbeeldingen van zulke objecten, dat wil zeggen zonder bewegingsonscherpte, kunnen verkregen worden met behulp van een korte en intense belichtingspuls. In hoofdstuk 3 wordt een techniek om een dergelijke puls te genereren geïntroduceerd welke gebaseerd is op laser geïnduceerde fluorescentie. Met deze methode wordt de voor beeldvormingsdoeleinden ongewenste coherentie van de laser sterk verminderd, terwijl de korte puls duur en hoge intensiteit behouden blijven.

De effectiviteit van deze belichtingsmethode wordt gedemonstreerd in hoofdstuk 4. In dit hoofdstuk wordt de oorzaak gezocht van de grote variaties die aanwezig zijn in het signaal van een glasvezel sonde hydrofoon bij het meten van de druk in een schokgolf. De afbeeldingen demonstreren het optreden van secundaire schokgolven als gevolg van cavitatie bel implosies. In het geval waarin cavitatie activiteit is onderdrukt door het toevoegen van azijnzuur aan de vloeistof, zijn de variaties in het signaal sterk verminderd. Dit suggereert dat de secundaire schokgolven de belangrijkste oorzaak zijn voor deze variaties.

Het onderzoek of en hoe cavitatie activiteit kan worden geïnitieerd uit microput belletjes wordt mogelijk gemaakt door de precieze controle over de dimensies en locatie gecombineerd met de beschikbare hoge snelheid opname technieken. In hoofdstuk 5 wordt het gedrag onderzocht van een $30 \mu \mathrm{m}$ diameter microput-bel in zowel een $80 \mathrm{kHz}$ als een $200 \mathrm{kHz}$ akoestisch veld. $\mathrm{Er}$ volgt dat boven een grenswaarde voor de drukamplitude, worden microbelletjes afgesplitst van het vloeistof-gas oppervlak. Dit proces is opmerkelijk stabiel. De bel-afsplitsing behelst geen nucleatie maar lijkt een gevolg te zijn van hoge amplitude capillaire golven welke het oppervlak lokaal vouwen tot er een singulariteit onstaat. De stabiliteit van het microbel productieproces vereist een 
continue op diffusie gebaseerd transport van gas terug de microput in.

Van beide frequenties is de respons van de gegenereerde microbellen door het akoestische veld het sterkst voor $200 \mathrm{kHz}$. Dit resulteert in een hemispherische cluster van cavitatiebellen gecentreerd boven de microput. Cavitatie activiteit kan verder versterkt worden met de introductie van meerdere microputjes. In dit geval resulteren secundaire Bjerknes krachten in de interactie van de clusters en de formatie van stabiele lintvormige cavitatie structuren. Een extra voordeel van de versterkte en gelocaliseerde cavitatie activiteit is dat het gedetailleerde studie mogelijk maakt van de oppervlakte cavitatie en de effecten ervan, zoals schokgolf emissie, schade vorming maar ook sonochemische reacties.

Sonochemie is een veelbelovende toepassing van cavitatie maar significante productie toename is vereist voordat het een wijdverbreide techniek kan worden. In hoofdstuk 6 wordt onderzocht of de door microputjes versterkte cavitatie activiteit ook resulteert in een hogere sonochemische efficiëntie. De productie van $\mathrm{OH}$ radicalen als gevolg van door cavitatie bel implosies geïnduceerde sonolyse is gekwantificeerd met behulp van terephtalische zuur dosimetrie. De resultaten laten zien dat de sonochemische efficiëntie daadwerkelijk toeneemt met ten minste een orde van grootte in vergelijking met de situatie zonder microputjes. Daarnaast blijkt uit afbeeldingen dat luminescentie als gevolg van de reactie van luminol met $\mathrm{OH}$ radicalen afkomstig is uit de zones van cavitatie activiteit.

Een van de meest gevestigde toepassingen gebaseerd op akoestische oppervlakte cavitatie is megasoon reinigen van halfgeleider substraten. De aanhoudende verkleining van de fragiele structuren maakt het efficiënt en schadeloos reinigen van deze substraten een bijzonder lastige uitdaging. In hoofdstuk 7 worden de cavitatiebelletjes onderzocht welke verantwoordelijk zijn voor reinigen of beschadigen van een oppervlak. Lokaliseren van deze belletjes is bewerkstelligd met behulp van een laag goud nanodeeltjes die zijn aangebracht op een transparant microscoop plaatje welke vervolgens loodrecht en bovenop een $1 \mathrm{MHz}$ staande golf is geplaatst. Zowel het reinigingsproces welke plaatsvindt op een lange tijdschaal als de sub-microseconde dynamica van de daarvoor verantwoordelijke belletjes zijn in top-view opgenomen. De resultaten laten zien dat in deze configuratie deeltjes worden verwijderd in zones die parallel lopen aan de nodale vlakken. Het verwijderen van deeltjes van het oppervlak is een gevolg van de krachten daarop uitgeoefend door geïsoleerde, snel over het oppervlak bewegende belletjes. De radiale oscillaties van deze bellen vertonen een subharmonische component. Deze component kan mogelijk toegeschreven worden aan asymmetrische bel oscillaties. 


\section{Acknowledgements}

Silent gratitude isn't much use to anyone

- Gladys Browyn Stern -

This thesis is the result of a research project carried out in the Physics of Fluids group of the Faculty of Science and Technology at the University of Twente and the Ultra Clean Processing Group at the Interuniversity MicroElectronics Center (IMEC), located in Leuven, Belgium. The financial support for this project was also provided by IMEC, for which I am very grateful.

$\mathrm{A} \mathrm{PhD}$ project has several basic ingredients. For example, on average it takes four years, it constitutes in an depth investigation and if possible contribution to a tiny fraction of science. And for its completion a report, called a thesis, is to be written. But in the end, it is the many great experiences and people involved which are most valuable and make up an unforgettable memory. I feel very grateful to have been able to do my $\mathrm{PhD}$ in two places which have abundantly provided the conditions for such a memory. The one where I spent most of my time was the outstanding Physics of Fluids group, led by Professor Detlef Lohse who I suspect has superpowers considering the amount of work he is able to do while guiding and greatly supporting his many $(\mathrm{PhD})$-students, postdocs and other staff members. I am very grateful for your trust, advice and motivation, in particular during the last few months.

I would also like to thank my former advisor and committee member, assist. Prof. Dr. Claus-Dieter Ohl, for enabling and motivating me to embark on this project, somewhat more than four years ago, but also for showing me that there is scientific value in almost every experiment.

A very special thanks goes to David Fernandez Rivas, the energetic Cuban guitar player and "bubble master" of the Mesoscale Chemical Systems group. I can honestly say that your enthusiastic presence and input revived my motivation to continue to study those small and pesky camera-dodging bubbles. I think the average of your "quick and dirty approach" and my delaying perfectionism resulted in some interesting and decent work. I feel very honored to have you as my paranymph.

Three other persons who deserve many thanks are my (former) office mates Rory Dijkink, Arjan van der Bos and Erik Gelderblom. It was great fun not only to share an office with you guys but also to be part of the iLIF-team. Working on this article was a very enjoyable and valuable experience, showing that shared interest and the right combination of individual qualities can be very effective. 
In the beginning of the project I naively set out to solve the "megasonic cleaning problem". Four years later and somewhat wiser I'm afraid this goal was not reached but I do feel privileged to have met, cooperated with- and learned from the many talented people of the UCP group at IMEC. I would therefore like to express my gratitude to Paul Mertens, Tom Janssens, Steven Brems, Marc Hauptmann, Geert Doumen, XiuMei Xu, Elisabeth Camerotto, Kurt Wostyn, Antoinne Pacco, Sandip Halder and Francesca Barbagini. It was a pleasure to work with you, experience your enthusiasm for high-speed movies of cavitation bubbles and your welcoming spirit both during and after working hours.

I thank Prof. Leen van Wijngaarden and Prof. Andrea Prosperetti naturally for being part of the committee but also for your help, contributions, suggestions with the theoretical work and other insightful moments. It is always a pleasure and at the same time somewhat humbling to see what a lifetime of scientific experience can do. I would also like to express my gratitude towards my copromotor Michel Versluis for your thorough and critical view and many useful suggestions on scientific content and how to present this. Your contributions during the last few months have been particularly helpful.

The Physics of Fluids group is blessed with an excellent technical staff. GertWim Bruggert and Martin Bos, many thanks for your high-quality custom made solutions. Bas Benschop thanks for your help on solving electronics and computer related issues. Joanita Leferink, I greatly appreciate the many administrative tasks you took care of even during the last months when my actions may have complicated these tasks somewhat.

I thank all my friends/colleagues at PoF for your good company, inspiration, support, laughs and discussions during work, coffee breaks, (preparing) group outings, summerschools, conferences, city trips, sailing trips, dinners, cycling, preparing sketches for promotions, pub quizzes, English poems recitals, watching rugby and so on. This is what great memories are made of!

Special thanks goes to Dinesh Chotoe, it is an honor to be your "white friend" and to have you by my side as a paranymph. Naturally it goes without saying that I am greatly thankful for having a wonderful and supporting family, though as said, silent gratitude isn't much use to anyone. And then there is gratitude beyond words; to my dearest Inge and Mette. 


\section{About the author}

Aaldert Zijlstra was born on August the 11th 1977 in Groningen, the Netherlands. After living in Gouda, Hoogeveen and Leerdam he graduated from high school "CSG Oude Hoven" in Gorinchem in 1995. The next destination was Enschede to start off with the study of Applied Physics at the University of Twente. In the following years several detours were made, including a 7 month stay at the Universite Joseph Fourier in Grenoble, France. In 2005 the study was picked up again with an internship at Thales/CNRS in Orsay, France on "Eddy current imaging using DigiSQUID". In 2007 he received his master degree in the Physics of Fluids group of Prof. Dr. Detlef Lohse under supervision of Dr. Claus-Dieter Ohl on the subject of "Jets from finite-wave free-surface reflections". Hereafter, the study of cavitation bubbles was given a four year extension with the project on "Megasonic Cleaning" in the same group and in cooperation with IMEC, Leuven. 
